PNL-2664

UC-70

\title{
Leaching of Fully Radioactive High-Level Waste Glass
}

D. J. Bradley

September 1978

Prepared for the U.S. Department of Energy under Contract EY-76-C-06-1830

Pacific Northwest Laboratory Operated for the U.S. Department of Energy by 
NOTICE

This report was prepared as an account of work sponsored by the United States Government. Neither the United States nor the Department of Energy, nor any of their employees, nor any of their contractors, subcontractors, or their employees, makes any warranty. express or implied, or assumes any legai liability or responsibility for the accuracy, completeness or usefuiness of any information, apparatus, product or process disclosed, or represents that its use would not infringe privateiy owned rights.

The views; opinions and conclusions contained in this report are those of the contractor and do not necessarily represent those of the United States Government or the United States Department of Energy.

\author{
PACIFIC NORTHWEST LABORATORY \\ operated by \\ BATTELLE \\ for the \\ UNITED STATES DEPARTMENT OF ENERGY \\ Under Contract EY-76-C-06-1830
}

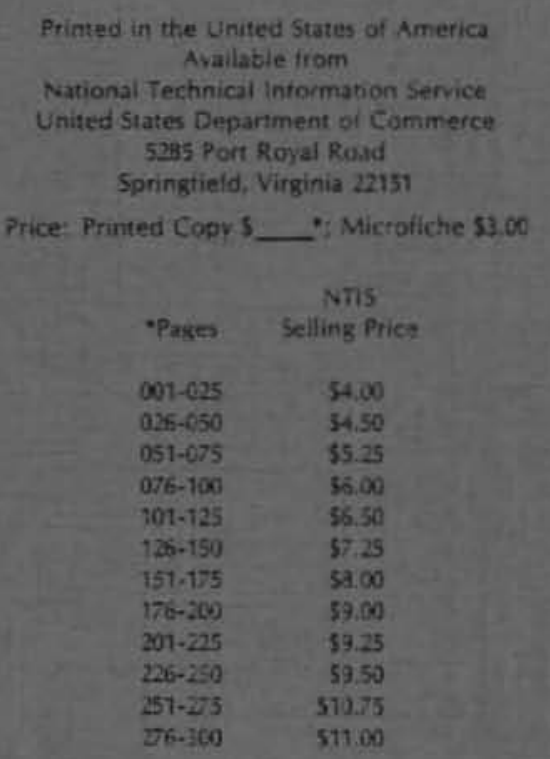


PNL -2664

UC -70

\section{3}

\section{LEACHING OF FULLY RADIOACTIVE HIGH-LEVEL WASTE GLASS}

D. J. Bradtey

September 1978

Prepared for the U.S. Department of Energy Under Contract EY-76-C-06-1830 


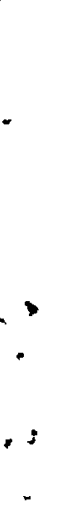

.
.

$\because$ 


\section{SUMMARY}

As part of continuing Department of Energy (DOE)-sponsored studies in waste management, the Pacific Northwest Laboratory (PNL) has been conducting the High-Level Waste Immobilization Program. The purpose of this program is to develop and demonstrate technology for incorporating nuclear wastes into final waste forms. This report describes the preparation and leach testing of fully radioactive, zinc borosilicate glass, which was prepared from power reactor waste.

Leach testing using the International Atomic Energy Association (IAEA) procedure was performed in deionized water for a period of 1.75 years. Leach rates were determined for activation products, fission products, and actinides. These rates ranged from $4 \times 10^{-5} \mathrm{~g}$ of $\mathrm{glass} / \mathrm{cm}^{2}$-day, based on cesium, to $4 \times 10^{-9} \mathrm{~g}$ of glass $/ \mathrm{cm}^{2}$-day, based on cerium. Following is the ranking of the release rates of the elements, from highest to lowest:

$$
\mathrm{Cs}>\mathrm{Sr}>\mathrm{Co}>\mathrm{Sb}>\mathrm{Mn}>\mathrm{Pu}>\mathrm{Eu}>\mathrm{Rh}>\mathrm{Cm}>\mathrm{Ce} \text {. }
$$

A similar leach test, using the same glass composition but with nonradioactive elements, has recently been completed. The leach rates of $\mathrm{Cs}$ and $\mathrm{Sr}$ for the nonradioactive glass were found to be in close agreement with those in this study.

Slopes calculated from curves of cumulative fractions leached show that radioisotope release begins with a diffusion-type mechanism and changes gradually to a silicate lattice alteration mechanism. Changes in sampling frequency altered the apparent release mechanism when leachant changes were longer than one month. The leach rates were quite constant for samples taken from the top to the bottom of the giass meli, indicating a homogeneous product.

Safety assessment studies and modeling programs use leach rates to predict the amount of radioactive material released should the waste be contacted by aqueous solutions. Further tests, focusing on geologic storage conditions and using fully radioactive wastes, are planned. 
. 


\section{CONTENTS}

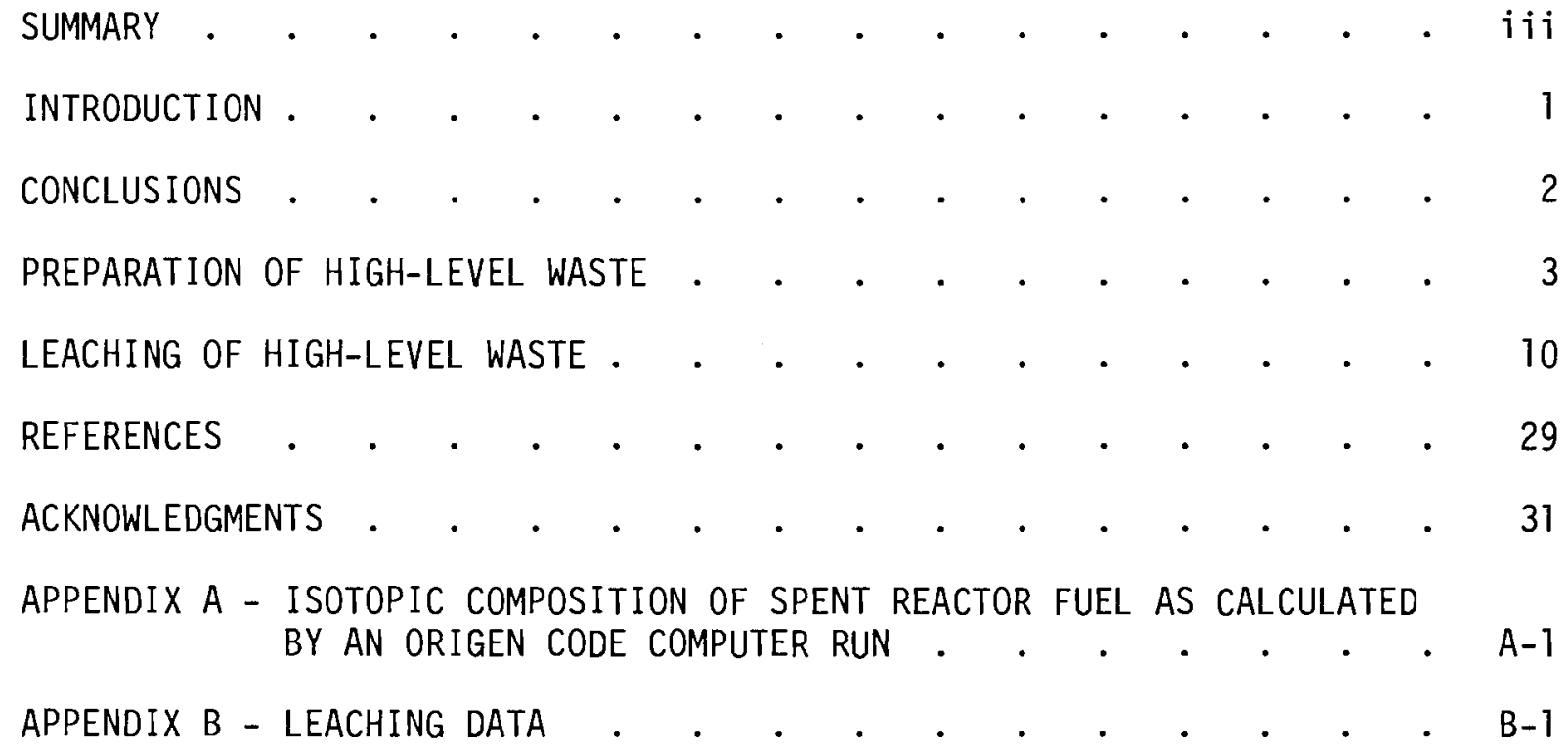




\section{FIGURES}

1 High-Level Waste Glass Melting Apparatus . . . . . . . 7

2 Sectioning of High-Level Waste Glass Canister. . . . . . 7

3 Photographs of High-Level Waste Glass Sections . . . . . 9

4 Long Term Leaching Apparatus of IAEA Test. . . . . . . 10

5 Leach Rate as a Function of Time, Top Section. . . . . . 14

6 Leach Rate as a Function of Time, Upper-Middle Section . . . 16

7 Leach Rate as a Function of Time, Bottom Section . . . . . 18

$8 \quad 5$ each Rate as a Function of Time and Section for

9 Legch Rate as a Function of Time and Section for ${ }^{154} \mathrm{Eu}$ and 21

10 Cumulative Fractions Leached as a Function of Time, Top Section . . . . . . . . . . . . . . 22

11 Cumulative Fractions Leached as a Function of Time, Bottom Section . . . . . . . . . . . . . 23 


\section{TABLES}

1 Characteristics of Spent Power Reactor Fuel . . . . . . 3

2 Comparison Between ORIGEN Value and Actual Abundance

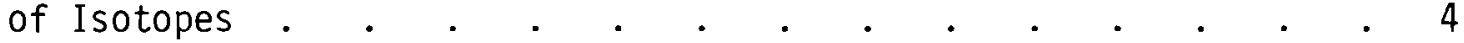

3 Fully Radioactive Waste Glass Composition . . . . . . 5

4 Preparation of High-Level Waste Glass. . . . . . . . . 6

5 Characteristics of High-Level Waste Glass Sections . . . . 8

6 Analys is of Hot Cell Deionized Water . . . . . . . . 11

7 High-Level Waste Leach Solution Sampling Schedule. . . . . 12

8 Element Release Fractions After 639 Days . . . . . . . 24

9 Slopes Calculated from Curves of Cumulative Fractions Leached. . 26

10 Slopes of Initial and Long-Term Release Mechanisms . . . . 27 


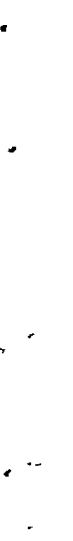




\section{INTRODUCTION}

Release rate data for radionuclides from fully radioactive waste forms are needed to evaluate the safety of nuclear waste glass. Presently, contact with water is considered the most important release path; therefore, the release properties of waste glass in water are of primary concern.

This work on high-level waste glasses was begun in 1975, before the change in Administration policy that deferred commercial nuclear reactor fuel reprocessing. However, this work has broad applications for understanding the properties of waste glass. Leach tests must be conducted on actual nuclear waste glass to validate the work on simulated waste glasses. Data is also needed on elements not included in simulated waste glasses. Only by testing the fully radioactive glasses can the full spectrum of radioisotopes be studied.

This report examines the leach testing of fully radioactive high-level waste glass developed at the Pacific Northwest Laboratory (PNL), operated by Battelle Memorial Institute for the Department of Energy (DOE). This glass, having a low sodium and a high fission product content, is called the reference composition glass.

High-level waste was generated by separating out the fission product and actinide (including about $90 \%$ of the uranium and plutonium) fractions from power reactor fuel irradiated to 55,000 MWd/MTU. The liquid waste was batch calcined, combined with a glass frit, and melted in a stainless steel crucible to prepare waste glass in approximately $100-\mathrm{g}$ batches. Leach tests were conducted on this fully radioactive reference glass to:

- determine the release rates of radioactive material from the glass,

- compare leach rates to those of nonradioactive waste of the same composition, and

- recommend further studies needed in this area. 


\section{CONCLUSIONS}

The release rates of 10 radioisotopes from fully radioactive waste glass, in deionized water for a period of 1.75 years, were determined. For cesium and strontium, good agreement exists between the leach rates for simulated and fully radioactive glass of the same composition.

It was shown that the release rate mechanism is dependent on time, sampling frequency, and type of element. For this study, only sampling intervals greater than one month had significant impact on the leach rate. Over the long testing period, two different release mechanisms occurred. This is consistent with previously reported observations.

Special areas needing additional work were identified, as follows:

- the effects of sample size and radiation on leaching

- the release mechanisms of radioactive material from glass

- the release rates of actinides and other elements from actual radioactive waste forms; the release rates of these elements must be measured under conditions of simulated geologic storage. 


\section{PREPARATION OF HIGH-LEVEL WASTE}

In July 1975, $2764 \mathrm{~g}$ of spent irradiated power reactor fuel were reprocessed in the hot cell facilities at PNL. Characteristics of this fuel are listed in Table 1. After dissolution of the fuel and extraction of uranium and plutonium, the liquid waste was boiled down, and then dried at $500^{\circ} \mathrm{C}$ for $1 \mathrm{hr}$ to yield $380 \mathrm{~g}$ of fine granular material, called calcine. A sample of this calcine was then analyzed for isotopic contents. An ORIGEN ${ }^{(1)}$ code computer run was made, which calculated the isotopic inventory in the waste, given the fuel's irradiation and decay history, and the uranium and plutonium separation history.

Table 2 compares (on the basis of curies) the quantitites of isotopes predicted by ORIGEN and measured by the above analysis. The agreement is quite good for all isotopes except ${ }^{106} \mathrm{Ru},{ }^{125} \mathrm{Sb}$, and ${ }^{134} \mathrm{Cs}$. The quantities of these isotopes were all lower than predicted by ORIGEN. For ruthenium and antimony, this difference might have been due to partial losses during the fuel dissolving operation. The reason for the $10 \mathrm{w}{ }^{134} \mathrm{Cs}$ value is not known, since a low value was not reflected in the ${ }^{137}$ Cs value, where the agreement was excellent. Note that manganese and cobalt, neutron activation products of fuel assemblies and fuel dissolving equipment, were also seen in the calcine and the waste glass, although they were not computed in this ORIGEN run.

\section{TABLE 1. Characteristics of Spent Power Reactor Fuel}

Enrichment $=5.81 w t \% u^{235}$

Theoretical Density $=93.6 \%$ of theoretical

Effective Fuel Power Hours $=27,800 \mathrm{hr}$

Initial Irradiation Date $=$ Dec. 1968

Discharge Date $=$ Oct. 1973

Average Burnup $=54,550 \mathrm{MWd} / \mathrm{MTU}$

Peak Burnup $=60,100 \mathrm{MWd} / \mathrm{MTU}$ 


\section{TABLE 2. Comparison Between ORIGEN Value and Actual Abundance of Isotopes(a)}

\begin{tabular}{|c|c|c|c|}
\hline Isotope & $\begin{array}{l}\text { Measured (b) Value, } \\
\mathrm{Ci} \text { in } 380 \mathrm{~g} \text { Calcine }\end{array}$ & $\begin{array}{l}\text { ORIGEN } \\
\text { Value, Ci in } \\
2764 \mathrm{~g} \text { Fuel }\end{array}$ & $\begin{array}{l}\text { Ratio of } \\
\text { Measured Ci to } \\
\text { ORIGEN Curies } \\
\end{array}$ \\
\hline${ }^{90} \mathrm{Sr}$ & 418 & 359 & 1.16 \\
\hline${ }^{106} \mathrm{Ru}$ & 25.1 & 268 & 0.094 \\
\hline${ }^{125} \mathrm{Sb}$ & 3.8 & 15.8 & 0.24 \\
\hline${ }^{134}$ Cs & 194 & 525 & 0.37 \\
\hline${ }^{137} \mathrm{Cs}$ & 456 & 470 & 0.97 \\
\hline${ }^{144} \mathrm{Ce}$ & 418 & 332 & 1.26 \\
\hline${ }^{154} \mathrm{Eu}$ & 29.6 & 33.2 & 0.89 \\
\hline${ }^{155_{\mathrm{Eu}}}$ & 16.7 & 13.3 & 1.26 \\
\hline $239+240 \mathrm{Pu}(\mathrm{c})$ & $\begin{array}{c}0.14 \\
\text { (1.65 before } \\
\text { reprocessing) }\end{array}$ & 2.3 & 0.72 \\
\hline${ }^{242} \mathrm{Cm}$ & 13.7 & 14.9 & 0.92 \\
\hline${ }^{244} \mathrm{Cm}$ & 4.9 & 2.7 & 1.81 \\
\hline
\end{tabular}

(a) Measured and ORIGEN values are decay-corrected to the same time.

(b) Takes into consideration volatilization losses measured during boil-down of liquid high-level waste.

(c) Analysis of the high-level liquid waste after fuel dissolution yielded $1.65 \mathrm{Ci}$ of $239+240 \mathrm{Pu}$ present. After separation, only $0.14 \mathrm{Ci}$ remained. Thus, the separation loss for $239+240 \mathrm{Pu}$ is $8.5 \%$. For uranium, similar analys is shows a $9 \%$ separation loss.

A detailed listing of the composition of the spent fuel, as output from an ORIGEN run, is given in Appendix $A$.

A zinc borosilicate glass frit was blended with waste calcine in a $3: 1$ weight ratio to make the waste glass. The composition of the product glass, based on frit composition, ORIGEN, and measured values, is given in Table 3.

A cylindrical melting container of $304 \mathrm{~L}$ stainless steel, $3.8 \mathrm{~cm} \times 12.7 \mathrm{~cm}$, with a wall thickness of $0.165 \mathrm{~cm}$, was used for the glass-making run. Table 4 gives the details of this glass run and Figure 1 shows the glass melting apparatus. 
TABLE 3. Fully Radioactive Waste Glass Composition

\begin{tabular}{|c|c|}
\hline Component & Weight, \% \\
\hline $\mathrm{SiO}_{2}$ & 27.8 \\
\hline $\mathrm{ZnO}$ & 21.7 \\
\hline $\mathrm{B}_{2} \mathrm{O}_{3}$ & 11.3 \\
\hline $\mathrm{UO}_{2}$ & 10.3 \\
\hline $\mathrm{Na}_{2} \mathrm{O}$ & 4.1 \\
\hline $\mathrm{K}_{2} \mathrm{O}$ & 4.1 \\
\hline Sro & 1.9 \\
\hline $\mathrm{ZrO}_{2}$ & 1.6 \\
\hline $\mathrm{MoO}_{3}$ & 1.6 \\
\hline $\mathrm{MgO}$ & 1.5 \\
\hline $\mathrm{CaO}$ & 1.5 \\
\hline $\mathrm{Ba} 0$ & 1.5 \\
\hline $\mathrm{Nd}_{2} \mathrm{O}_{3}$ & 1.5 \\
\hline $\mathrm{CeO}_{2}$ & 1.0 \\
\hline $\mathrm{Cs}_{2} \mathrm{O}$ & 0.8 \\
\hline $\mathrm{BaO}$ & 0.5 \\
\hline $\mathrm{La}_{2} \mathrm{O}_{3}$ & 0.45 \\
\hline $\mathrm{Pr}_{2} \mathrm{O}_{3}$ & 0.44 \\
\hline PdO & 0.43 \\
\hline $\mathrm{TC}_{2} \mathrm{O}_{7}$ & 0.39 \\
\hline $\mathrm{Sm}_{2} \mathrm{O}_{3}$ & 0.29 \\
\hline $\mathrm{NpO}_{2}$ & 0.22 \\
\hline $\mathrm{Y}_{2} \mathrm{O}_{3}$ & 0.19 \\
\hline $\mathrm{PuO}_{2}$ & 0.12 \\
\hline $\mathrm{RuO}_{2}$ & 0.09 \\
\hline $\mathrm{Eu}_{2} \mathrm{O}_{3}$ & 0.06 \\
\hline $\mathrm{AmO}_{2}$ & 0.06 \\
\hline $\mathrm{Gd}_{2} \mathrm{O}_{3}$ & 0.05 \\
\hline $\mathrm{COO}$ & $1.1 \times 10^{-4}$ \\
\hline \multirow[t]{2}{*}{$\mathrm{MnO}$} & $6.0 \times 10^{-6}$ \\
\hline & 95.5 \\
\hline
\end{tabular}

(a) The remaining material is made up of chemicals used in the fuel dissolution and uranium/plutonium separation operations. 
After $3 \mathrm{hr}$ at melt temperature, the furnace was shut off and the glass was allowed to cool at an average rate of $220^{\circ} \mathrm{C} / \mathrm{hr}$. The stainless steel canister was then sectioned into disks with a diamond saw. This same procedure, on subsequent glass making runs, provided samples of fully radioactive glass for metallography and electron microscopy. Information on these analyses, along with glass making volatization data, is given in BNWL-2252 ${ }^{(2)}$ and BNWL-2625. It was assumed that the preleaching effects of the diamond saw cutting operation would be negligible for a long-term leaching test.

Figure 2 depicts the sectioning of the melting container and Table 5 gives details on the resulting sections. Figure 3 shows three of the four sections used for this leaching study. The upper-middle section, not shown because of a camera exposure problem, appears identical to the bottom-middle section. The top section appears as a donut because the top of the melt is concave due to shrinkage. All sections appear homogeneous, without large cracks or voids. Prior to the start of the leach test, all wafers were washed in acetone to remove surface particles, and dried in air.

TABLE 4. Preparation of High-Level Waste Glass

$\begin{array}{lll}\text { Wt Calcine }+ \text { Frit } & = & 99.34 \mathrm{~g} \\ \text { Melt Temperature } & = & 1050^{\circ} \mathrm{C} \\ \text { Calcine Waste Loading } & = & 25 \% \\ \text { Time to Reach Melt } & & \\ \quad \text { Temperature } & 110 \mathrm{~min} \\ \text { Time at Melt } & & 180 \mathrm{~min} \\ \quad \text { Temperature } & = & 0.44 \mathrm{l} / \mathrm{min} \\ \text { Cover Gas Flow Rate } & = & 12 / 21 / 75 \\ \text { Date } & = & \end{array}$




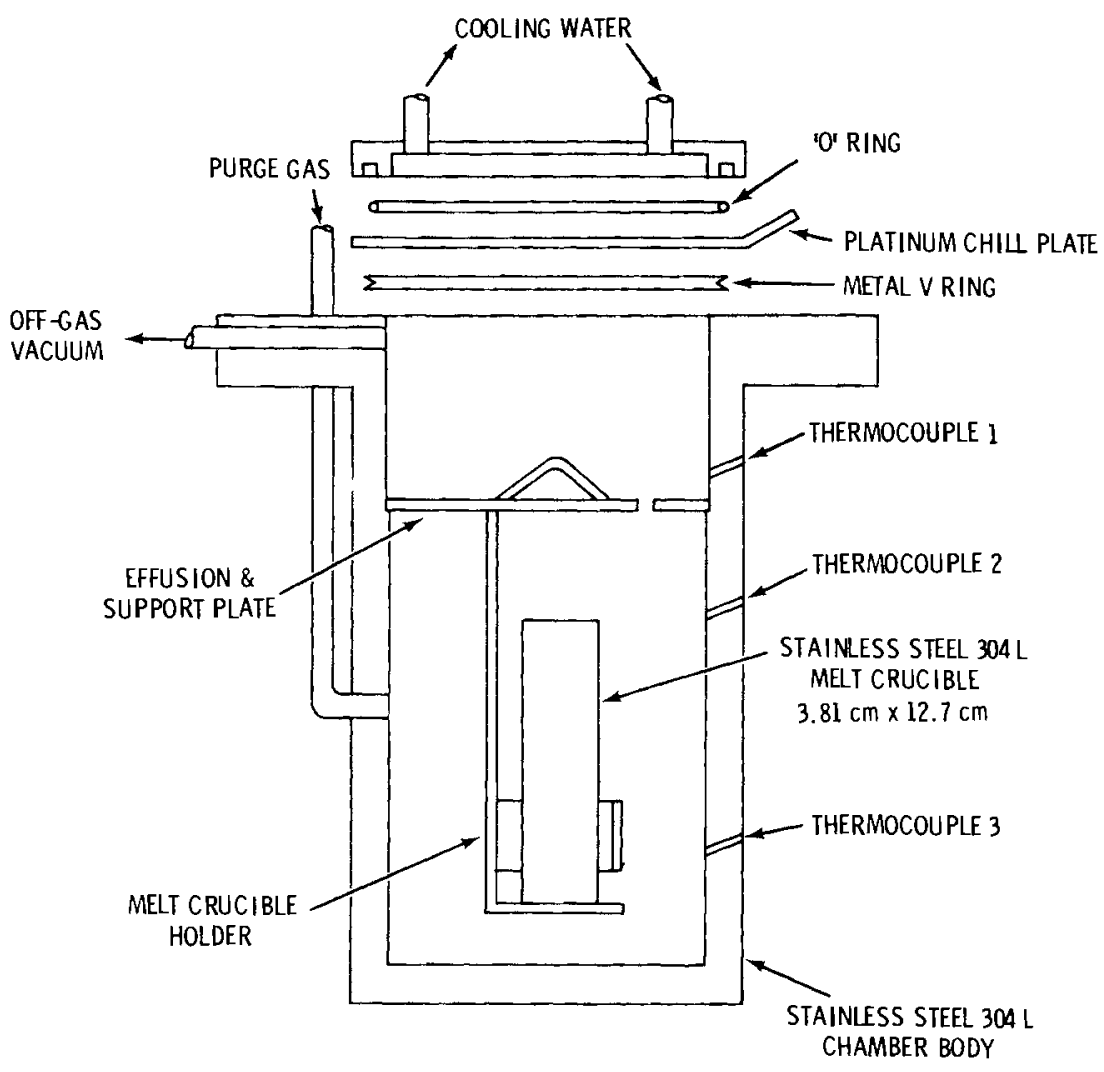

FIGURE 1. High-level Waste Glass Melting Apparatus

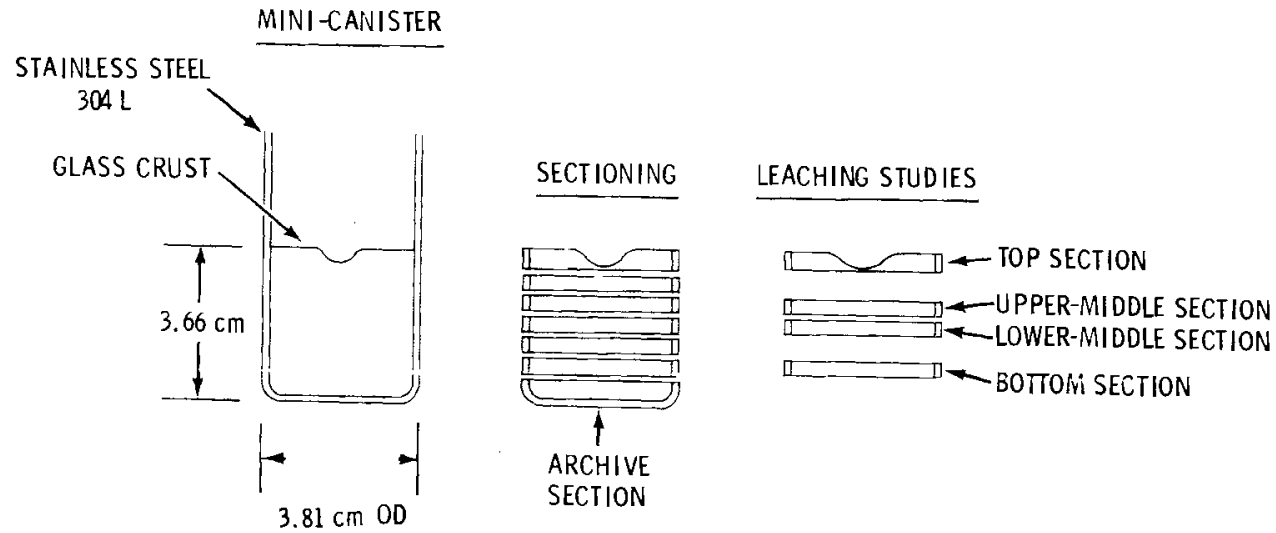

FIGURE 2. Sectioning of High-Leve1 Waste Glass Canister 
TABLE 5. Characteristics of High-Level Waste Glass Sections

\begin{tabular}{|c|c|c|c|c|c|}
\hline Section & $\begin{array}{l}\text { Total } \\
w t, g \\
\end{array}$ & $\begin{array}{c}\text { Thickness, } \\
\mathrm{cm}\end{array}$ & $\begin{array}{c}\text { Stainless } \\
\text { Steel Wt, g }\end{array}$ & $\begin{array}{l}\text { Glass } \\
W t, g \\
\end{array}$ & $\begin{array}{l}\text { Geometric } \\
\text { Glass Surface } \\
\text { Area, } \mathrm{cm}^{2} \\
\end{array}$ \\
\hline Bottom & 17.06 & 0.361 & 5.03 & 12.03 & 19.0 \\
\hline Lower-middle & 12.20 & 0.274 & 3.82 & 8.38 & 19.0 \\
\hline Upper-middle & 13.17 & 0.292 & 4.07 & 9.10 & 19.0 \\
\hline Top & 12.64 & 0.541 & 7.54 & 5.10 & 13.5 \\
\hline
\end{tabular}




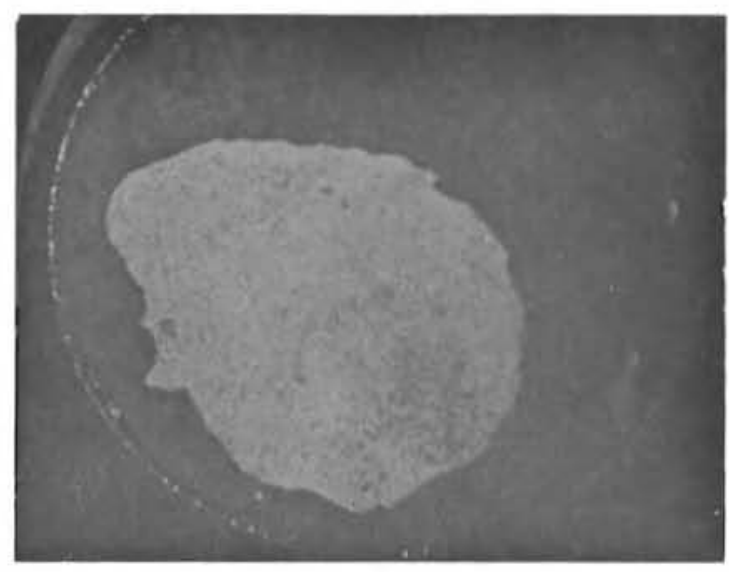

TOP SECTION

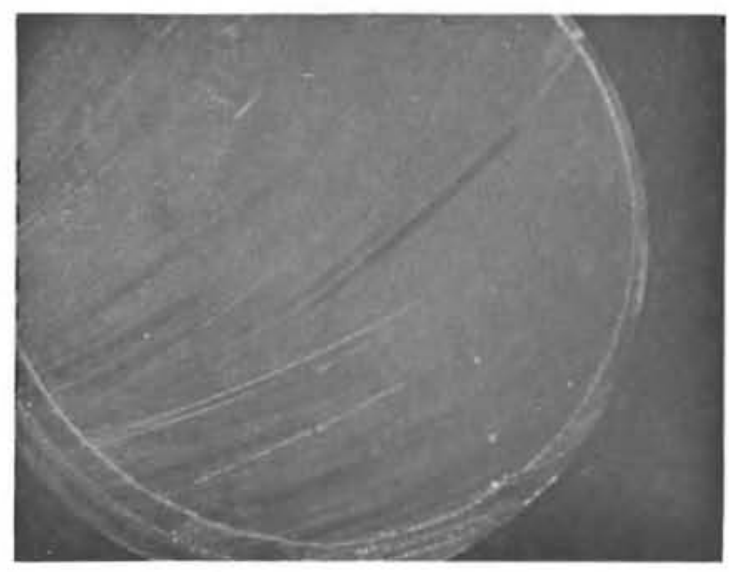

BOTTOM-

MIDDLE SECTION

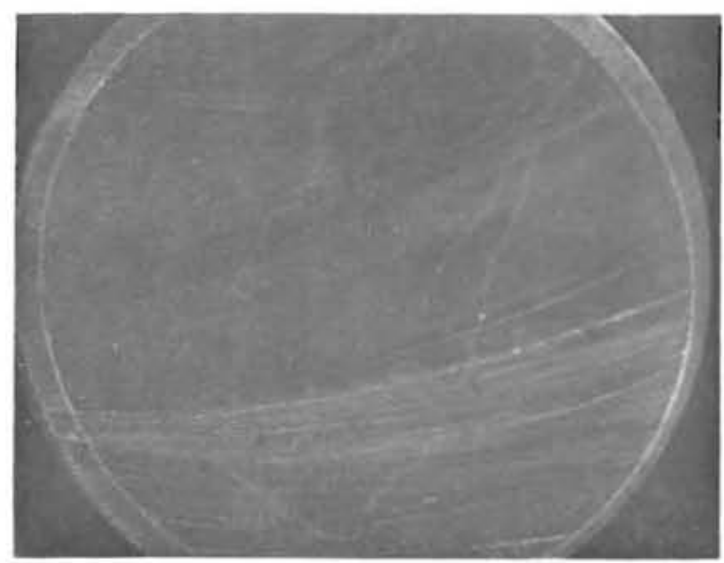

BOTTOM SECTION

FIGURE 3. Photographs of High-Level Waste Glass Sections 


\section{LEACHING OF HIGH-LEVEL WASTE}

The four high-level waste glass sections began leaching in February 1976. The leaching apparatus is depicted in Figure 4. The leachate was deionized water, and its composition after passing through a hot cell piping system is given in Table 6 . The ratio of leachate volume $(500 \mathrm{~m} \ell)$ to sample surface area was 26. The leachate was changed according to the International Atomic Energy Association (IAEA) ${ }^{(4)}$ procedure shown in Table 7 . On leachate changing day, a 10 me aliquot was removed from the container and acidified with concentrated nitric acid to a $\mathrm{pH}$ of 1 . This acid was added to prevent adherence of isotopes on the sample container walls. Analysis of the sample consisted of:

- gamma spectroscopy

- separation of cesium and strontium(5)

- recount by gamma spectroscopy

- beta counting of separated ${ }^{90} \mathrm{Sr}$

- alpha energy analysis.

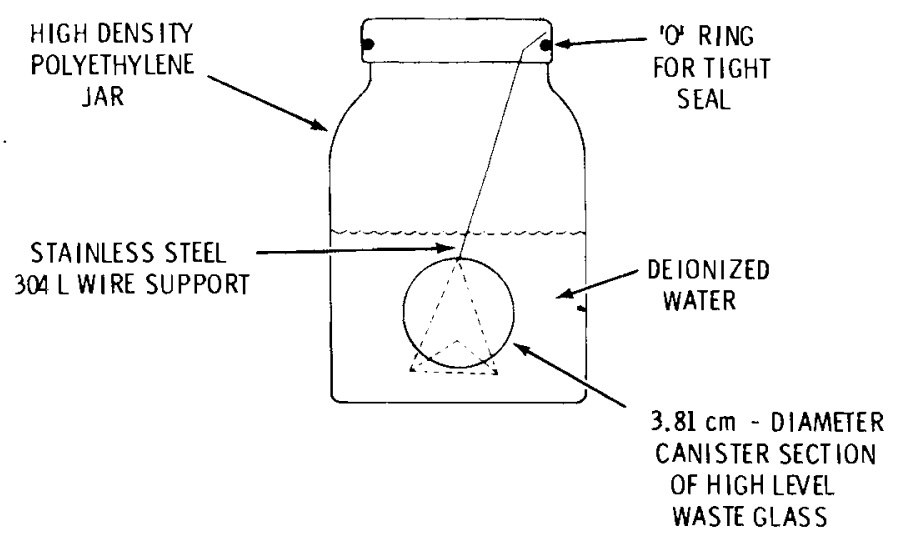

FIGURE 4. Long-Term Leaching Apparatus of IAEA Test 


$$
\begin{aligned}
& \text { TABLE 6. Analysis of Hot Cell } \\
& \text { Deionized Water } \\
& \text { - } \mathrm{pH}=6.1 \\
& \text { - Conductivity }=4.8 \mu \mathrm{mho} / \mathrm{cm} \\
& \text { - Eh }=497 \mathrm{mV} \\
& \text { - Elemental Analysis - Spark } \\
& \text { Source Mass Spectrographic } \\
& \text { Analysis }
\end{aligned}
$$

\begin{tabular}{|c|c|}
\hline Element & $\begin{array}{c}\text { ppm } \\
\text { ( } \pm \text { factor of } 3)\end{array}$ \\
\hline $\mathrm{Na}$ & 10.0 \\
\hline$K$ & 2.0 \\
\hline $\mathrm{A} 1$ & 2.0 \\
\hline$S$ & 1.0 \\
\hline $\mathrm{Fe}$ & 0.3 \\
\hline $\mathrm{Ca}$ & 0.2 \\
\hline $\mathrm{Cl}$ & 0.2 \\
\hline$F$ & 0.1 \\
\hline $\mathrm{Eu}$ & 0.1 \\
\hline $\mathrm{Be}$ & 0.05 \\
\hline
\end{tabular}
- Elemental Analysis - Spark Source Mass Spectrographic Analys is

Leach rates based on various isotopes were calculated by the equation: ${ }^{(6)}$

$$
R i=\frac{a_{0}}{A_{0} S t}
$$

where $\mathrm{Ri}=$ incremental leach rate, $\mathrm{g} / \mathrm{cm}^{2}$-day

$$
\begin{aligned}
a_{0}= & \text { activity of isotope in leachate, } \mathrm{sec}^{-1} \\
A_{0}= & \text { specific activity of isotope in sample, } \mathrm{sec}^{-1} \text {-gram }{ }^{-1} \text { (Based on } \\
& \text { measured activity of the high-level waste calcine; resulting glass } \\
& \text { assumed to be homogeneous. All activities } a_{0} \text { and } A_{0} \text { were decay } \\
& \text { corrected to the same time.) }
\end{aligned}
$$


$S=$ geometric surface area of sample, $\mathrm{cm}^{2}$

$t=$ leaching time, days.

TABLE 7. High-Level Waste Leach Solution Sampling Schedule

\begin{tabular}{|c|c|c|}
\hline Series No. & $\begin{array}{l}\text { Leaching } \\
\text { Time, Days }\end{array}$ & $\begin{array}{c}\text { Cummulative } \\
\text { Leach Time, } \\
\text { Days }\end{array}$ \\
\hline 1 & 1 Day & 1 \\
\hline 2 & 1 Day & 2 \\
\hline 3 & 1 Day & 3 \\
\hline 4 & 1 Day & 4 \\
\hline 5 & 4 Days & 8 \\
\hline 6 & 6 Days & 14 \\
\hline 7 & 7 Days & 21 \\
\hline 8 & 7 Days & 28 \\
\hline 9 & 7 Days & 35 \\
\hline 10 & 7 Days & 42 \\
\hline 11 & 7 Days & 49 \\
\hline 12 & 7 Days & 56 \\
\hline 13 & 30 Days & 86 \\
\hline 14 & 30 Days & 116 \\
\hline 15 & 34 Days & 150 \\
\hline 16 & 32 Days & 182 \\
\hline 17 & 29 Days & 211 \\
\hline 18 & 28 Days & 239 \\
\hline 19 & 182 Days & 421 \\
\hline 20 & 218 Days & 639 \\
\hline
\end{tabular}

Cumulative fraction leached was calculated by the equation: ${ }^{(6)}$

$$
\text { Cumulative Fraction }=\frac{\sum_{0}^{a_{0}}}{A_{0} W}
$$




$$
\text { where } \begin{aligned}
a_{0} & =\text { activity of isotope in leachate, } \mathrm{sec}^{-1} \\
A_{0} & =\text { specific activity of isotope in sample, } \mathrm{sec}^{-1} \text {-gram }^{-1} \\
W & =\text { sample weight, grams. }
\end{aligned}
$$

Figures 5 through 7 show plots of leach rate versus time for the top, uppermiddle, and bottom sections of high-level waste glass. [Parts (a) and (b) of each figure are for different groups of isotopes.] A11 isotopes have a higher leach rate at the beginning of the test and rapidly level off within one week.

The leach rates seen here can be compared to those from a similar test on simulated waste glass of the same composition. The leach rates for cesium and strontium from the fully radioactive glass were in very close agreement with the leach rates of cesium and strontium from the simulated glass. Leaching data on other elements from simulated glass are not available at this time. Although both tests were done on bulk glass samples, they differed in configuration. The fully radioactive samples were disks $[\sim 0.3 \mathrm{~cm}$ (height) $\mathrm{x}$ $3.5 \mathrm{~cm}$ (diameter)], and the simulated glass sample was a cylinder $[0.73 \mathrm{~cm}$ (height) $\times 0.98 \mathrm{~cm}$ (diameter)].

The size of the sample being leached has been found to have a large effect, up to a factor of 100 , on the apparent leach rate. (3) In contrast, radiation is believed to have a very small effect on leach rates; this has been shown for the case of alpha radiation. (7) Further studies are needed to show that this is the case for fully radioactive glass. 


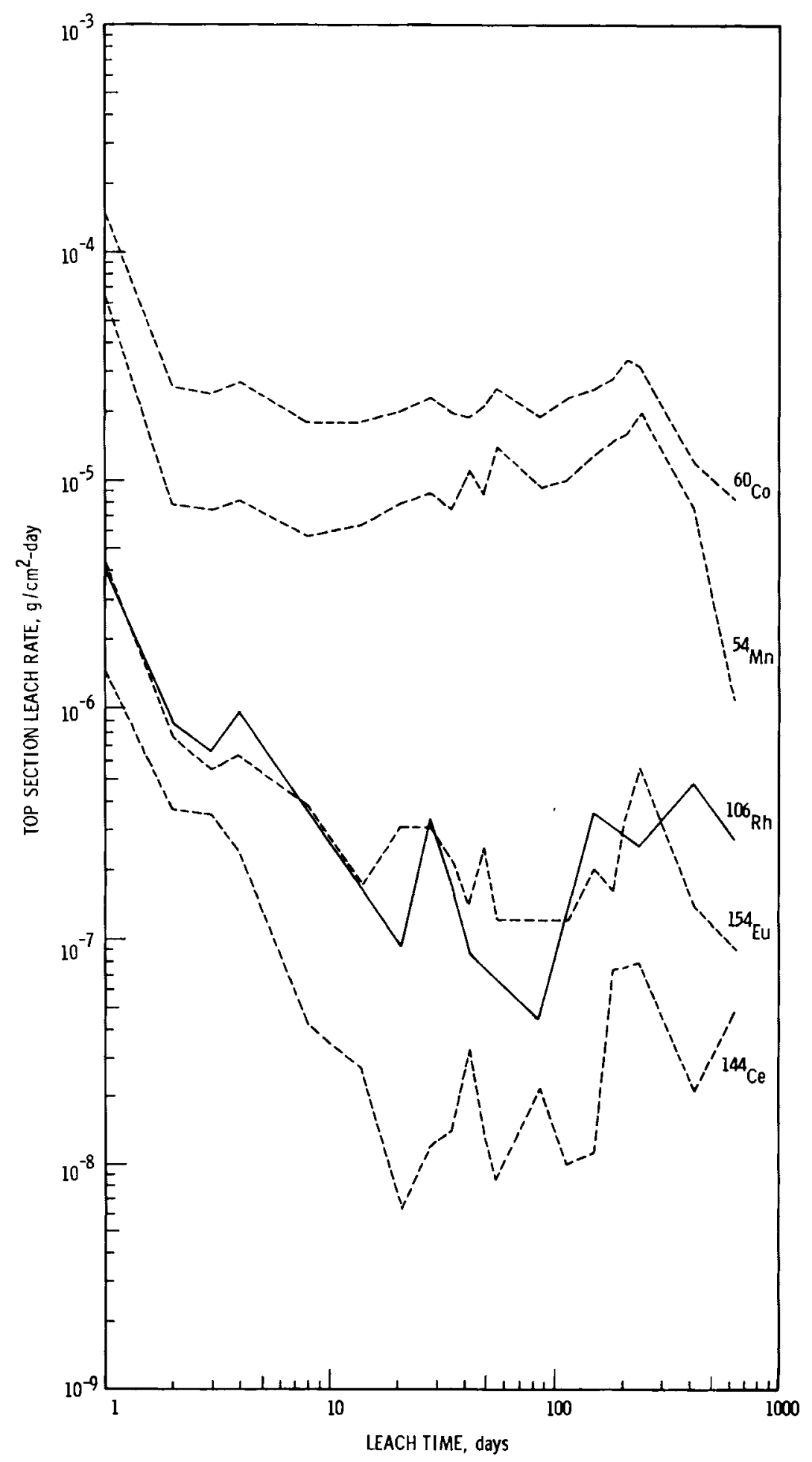

FIGURE 5a. Leach Rate as a Function of Time, Top Section 


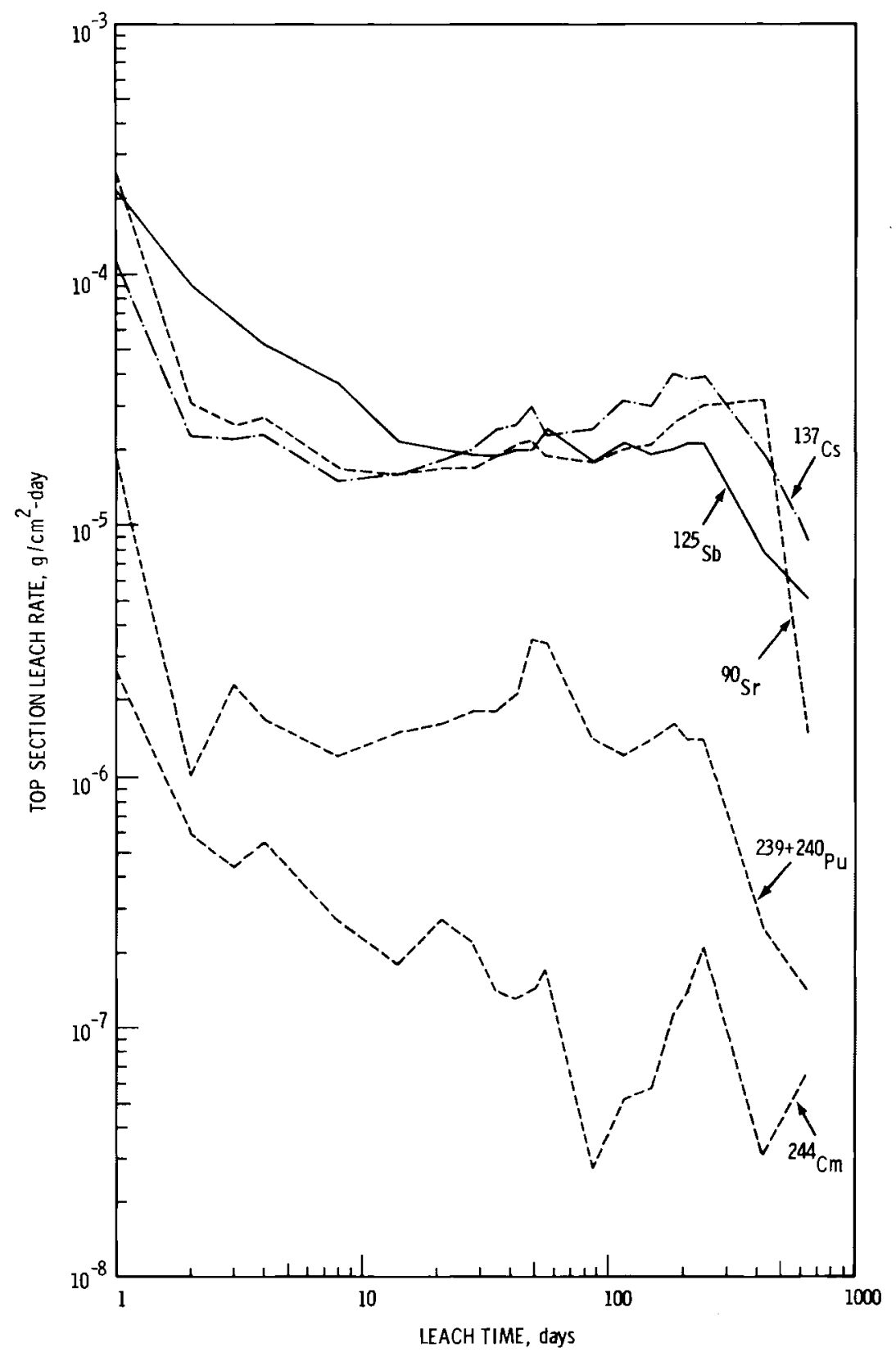

FIGURE 5b. Leach Rate as a Function of Time, Top Section 


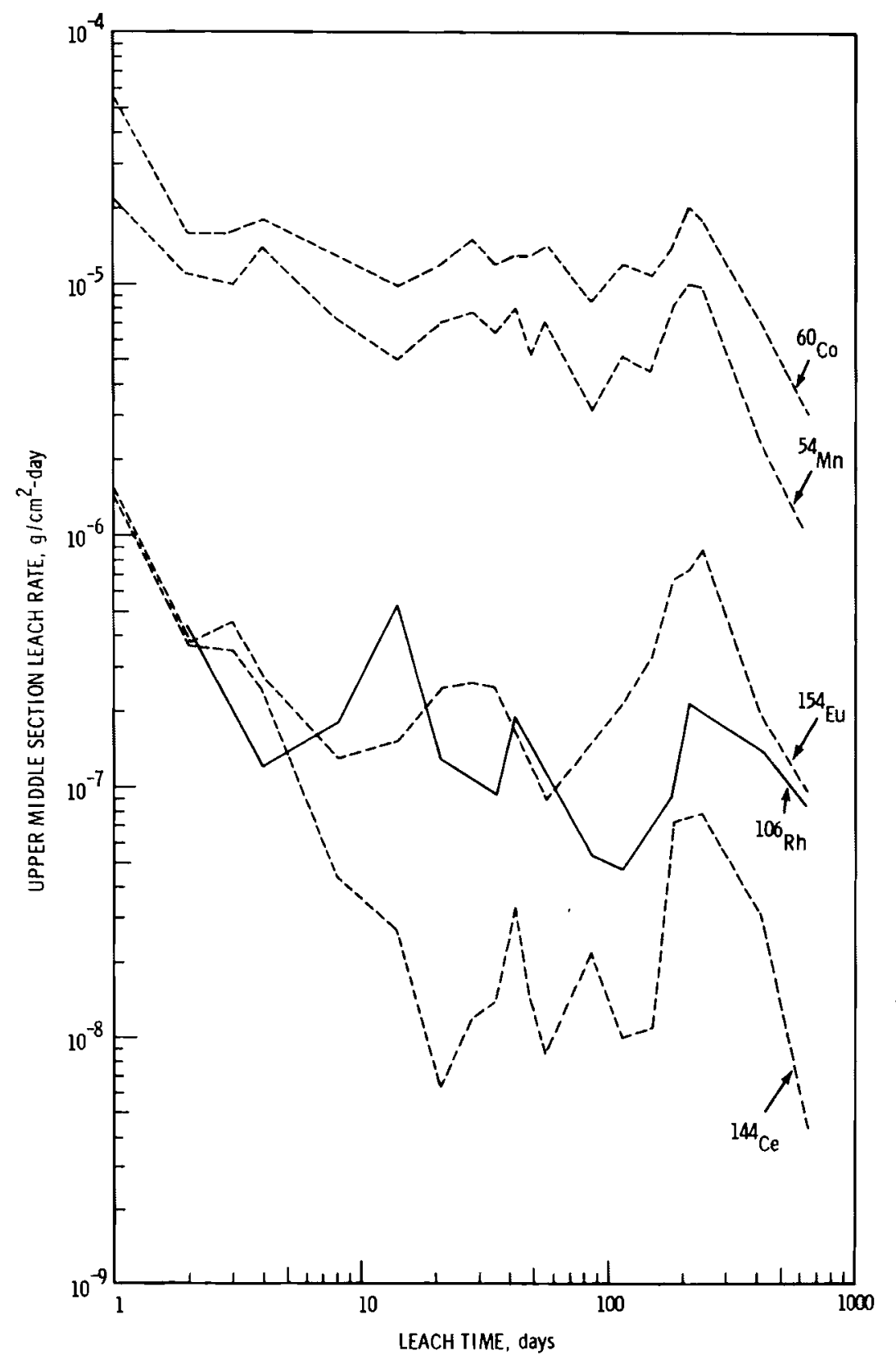

FIGURE 6a. Leach Rate as a Function of Time, Upper-Middle Section 


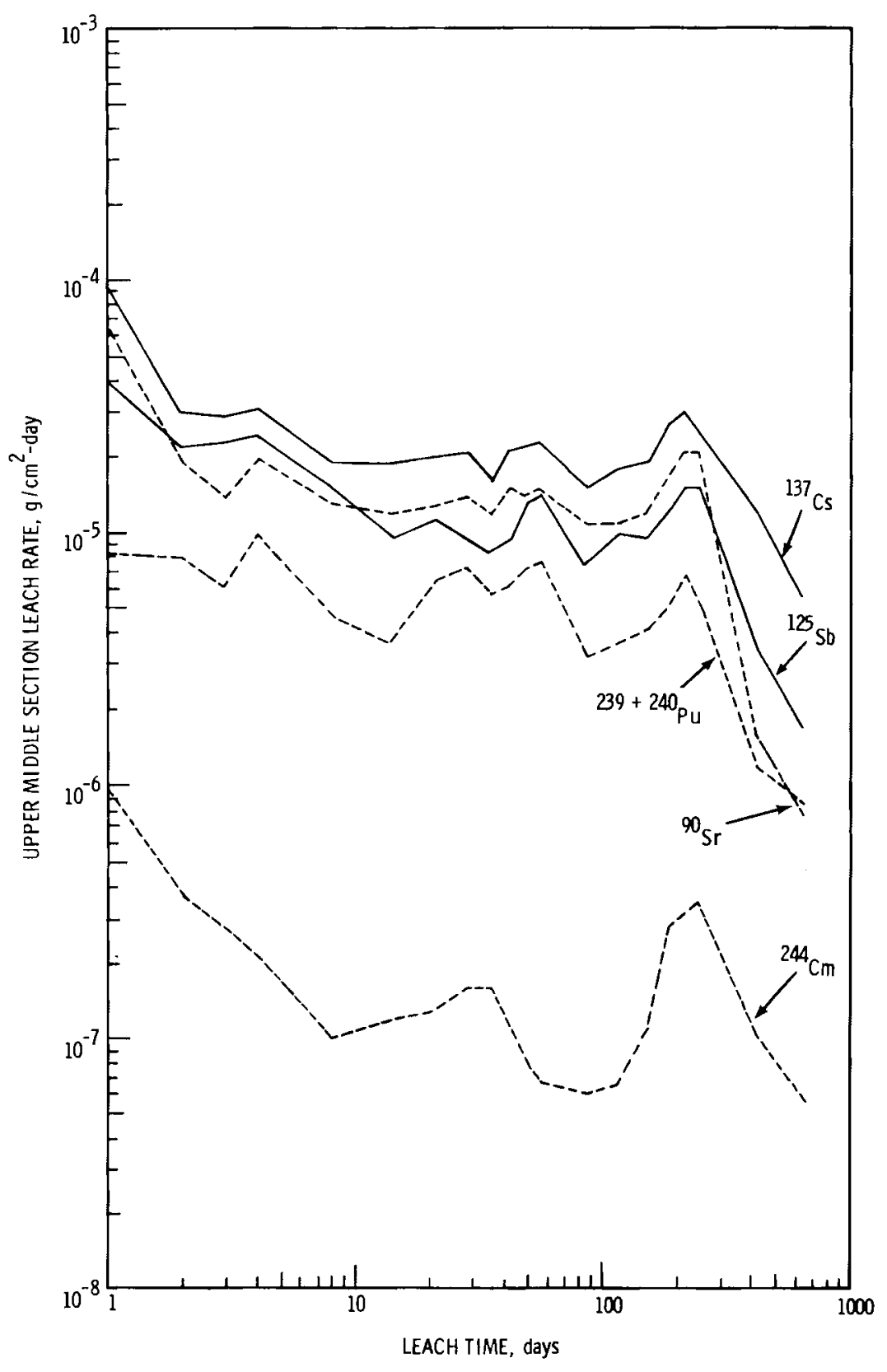

FIGURE 6b. Leach Rate as a Function of Time, Upper-Middle Section 


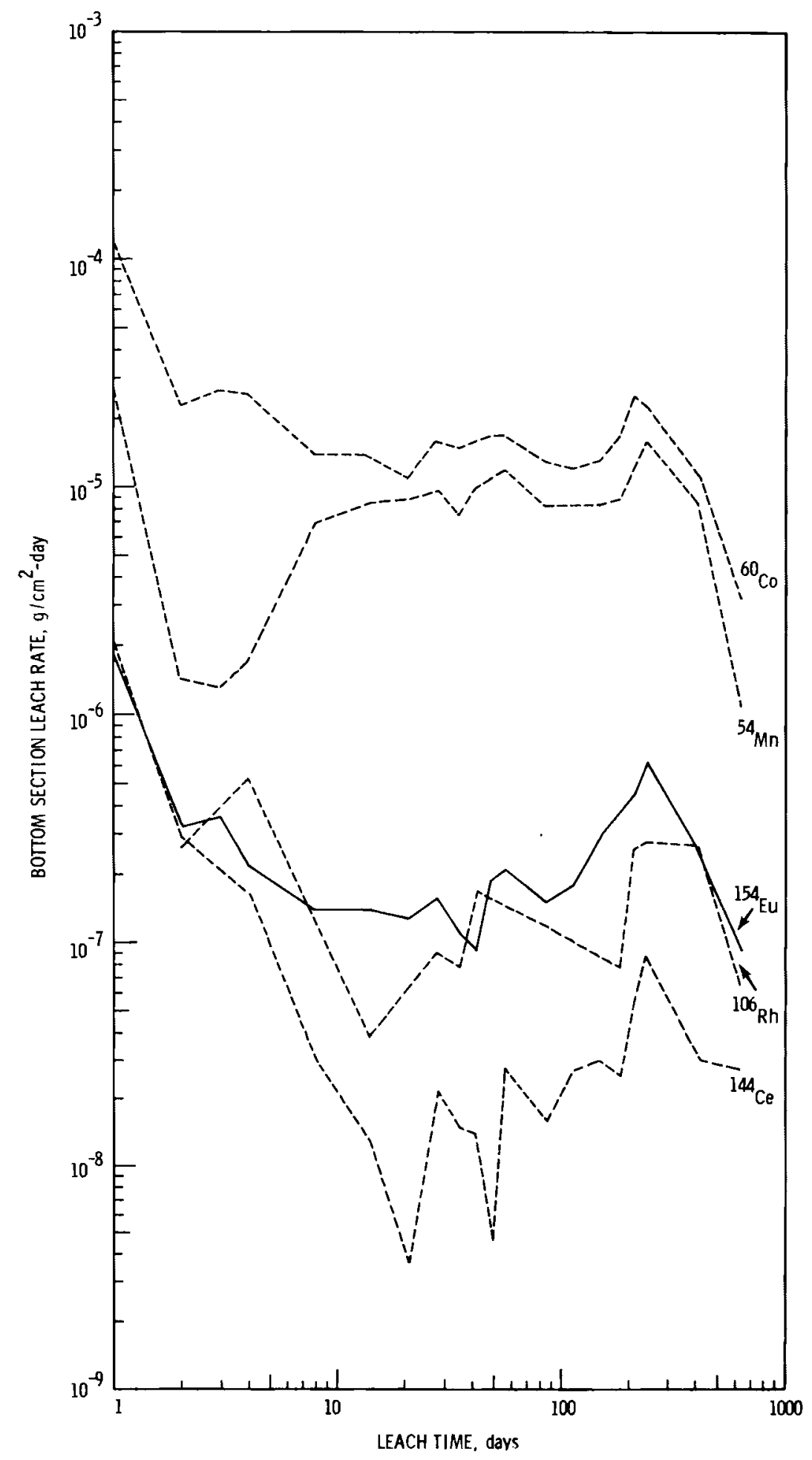

FIGURE 7a. Leach Rate as a Function of Time, Bottom Section 


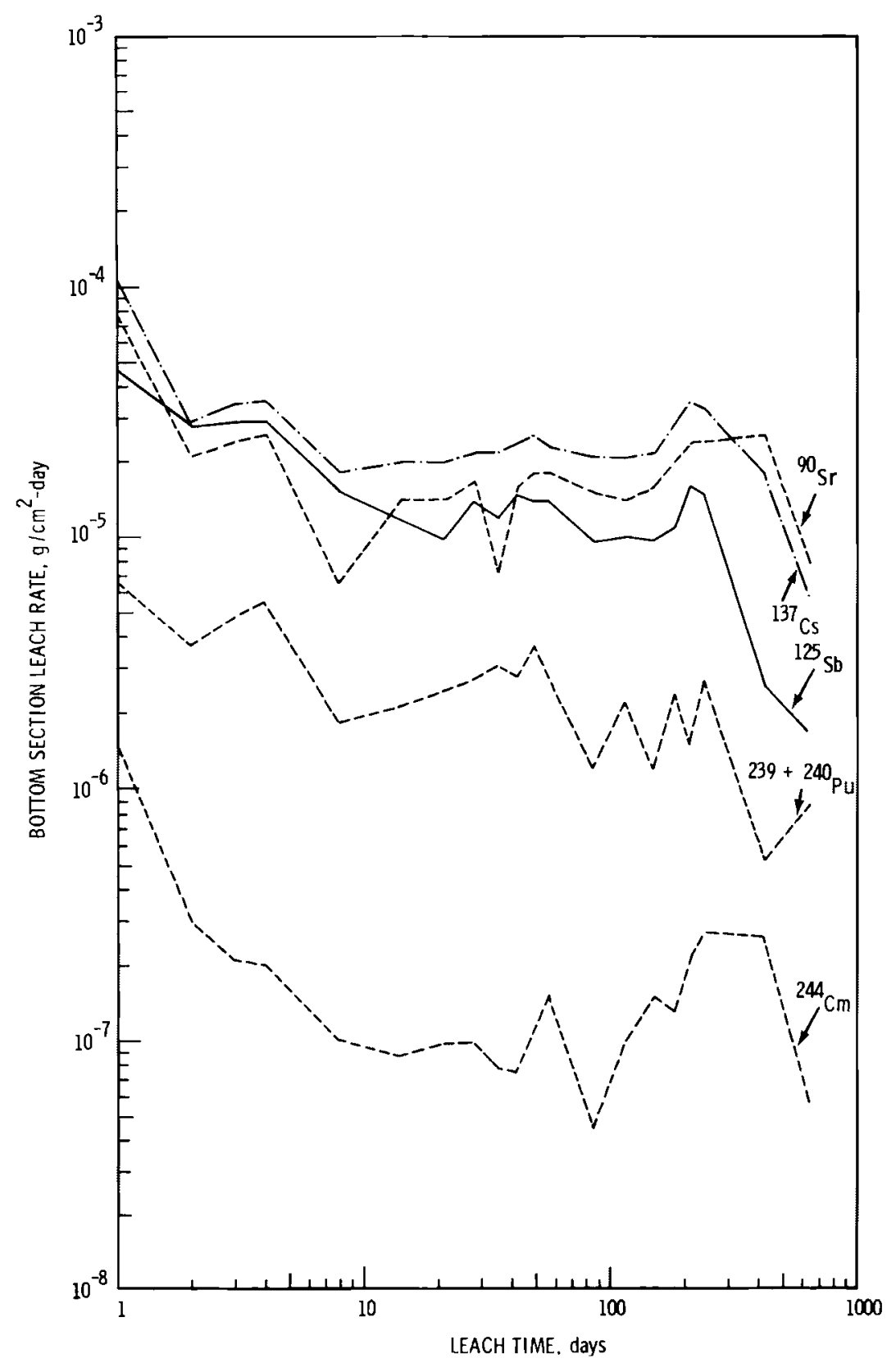

FIGURE 7b. Leach Rate as a Function of Time, Bottom Section 
Figures 8 and 9 show the leach rates of ${ }^{54} \mathrm{Mn},{ }^{137} \mathrm{Cs},{ }^{154} \mathrm{Eu}$, and $239+240 \mathrm{Pu}$ for each section. From these figures, it can be seen that the leach rates vary little from top to bottom of the waste canister. Although these four isotopes represent a wide range in mass and expected volatility, they indicate that there are no significant inhomogeneities in the glass column. This conclusion is supported by recent gamma spectroscopy on the high-level waste glass sections. (3) The widest deviation is seen in $239+240 \mathrm{Pu}$, which has a higher leach rate in the middle section.

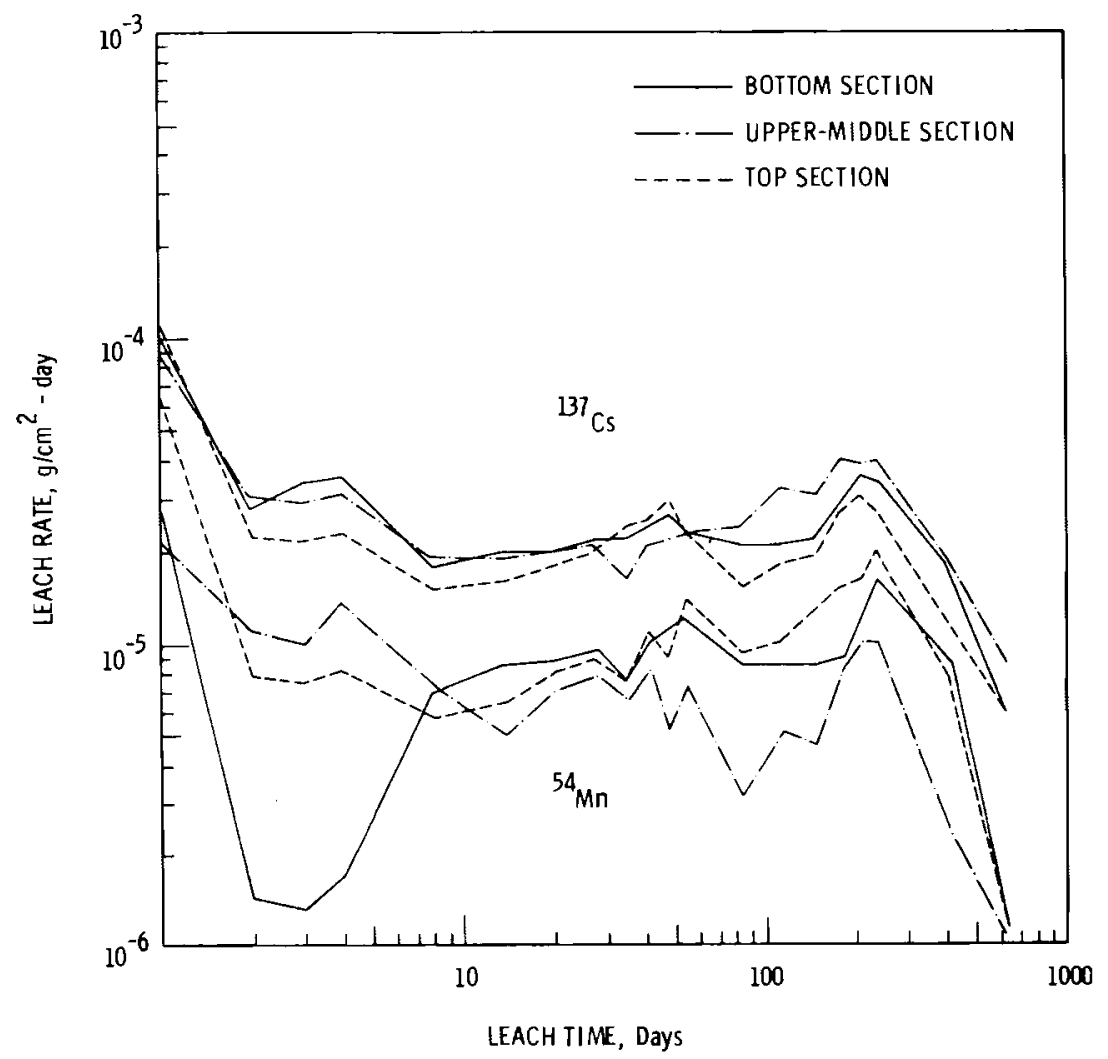

FIGURE 8. Leach Rate as a Function of Time and Section for $54 \mathrm{Mn}$ and $137 \mathrm{Cs}$ 


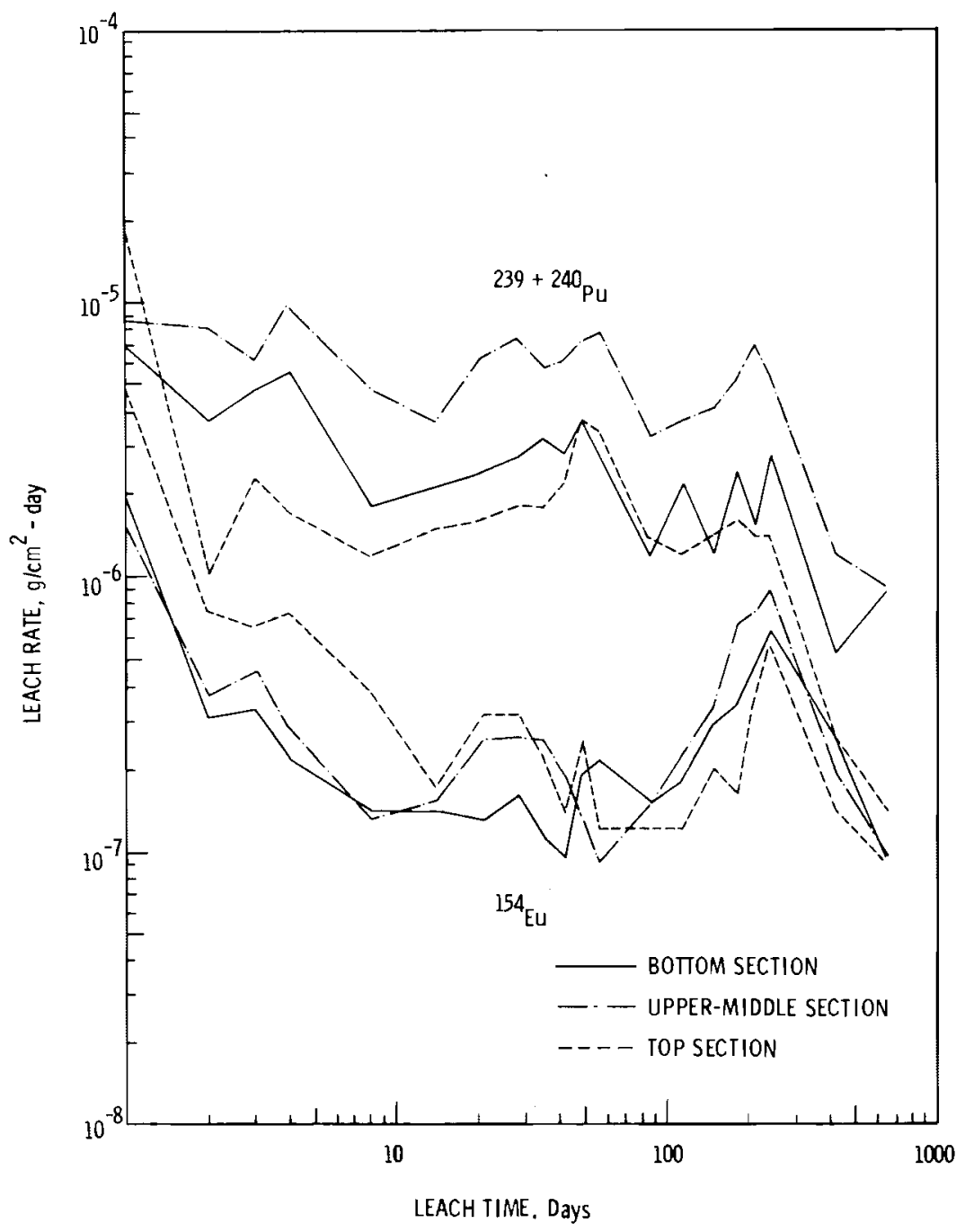

FIGURE 9. Leach Rate as a Function of Time and Section for 154Eu and $239+240 \mathrm{Pu}$

Curves showing the cumulative fractions leached were also calculated from the data and are shown for the top and bottom sections in Figures 10 and 11 , respectively. A complete set of ieaching data (including cumulative fractions leached) is given in Appendix B. From the curves of leach rate and cumulative fractions leached (and data in Appendix B) a ranking of the elements can be made with respect to their leachability. This ranking follows (on page 24) in Table 8. Because of the time and cost required for multiple actinide separations, analyses for only $\mathrm{Pu}$ and $\mathrm{Cm}$ were made. 


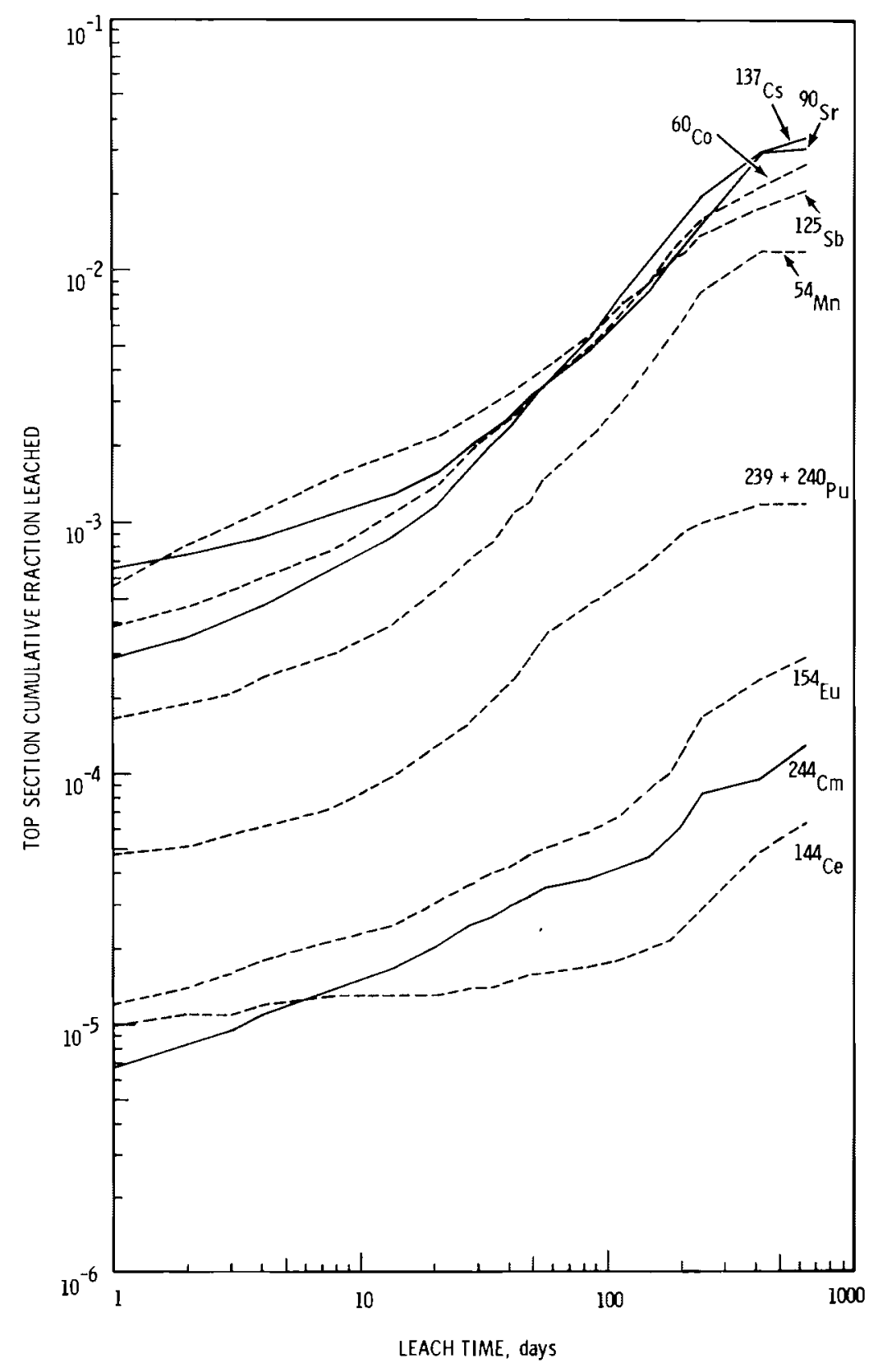

FIGURE 10. Cumulative Fractions Leached as a Function of Time, Top Section 


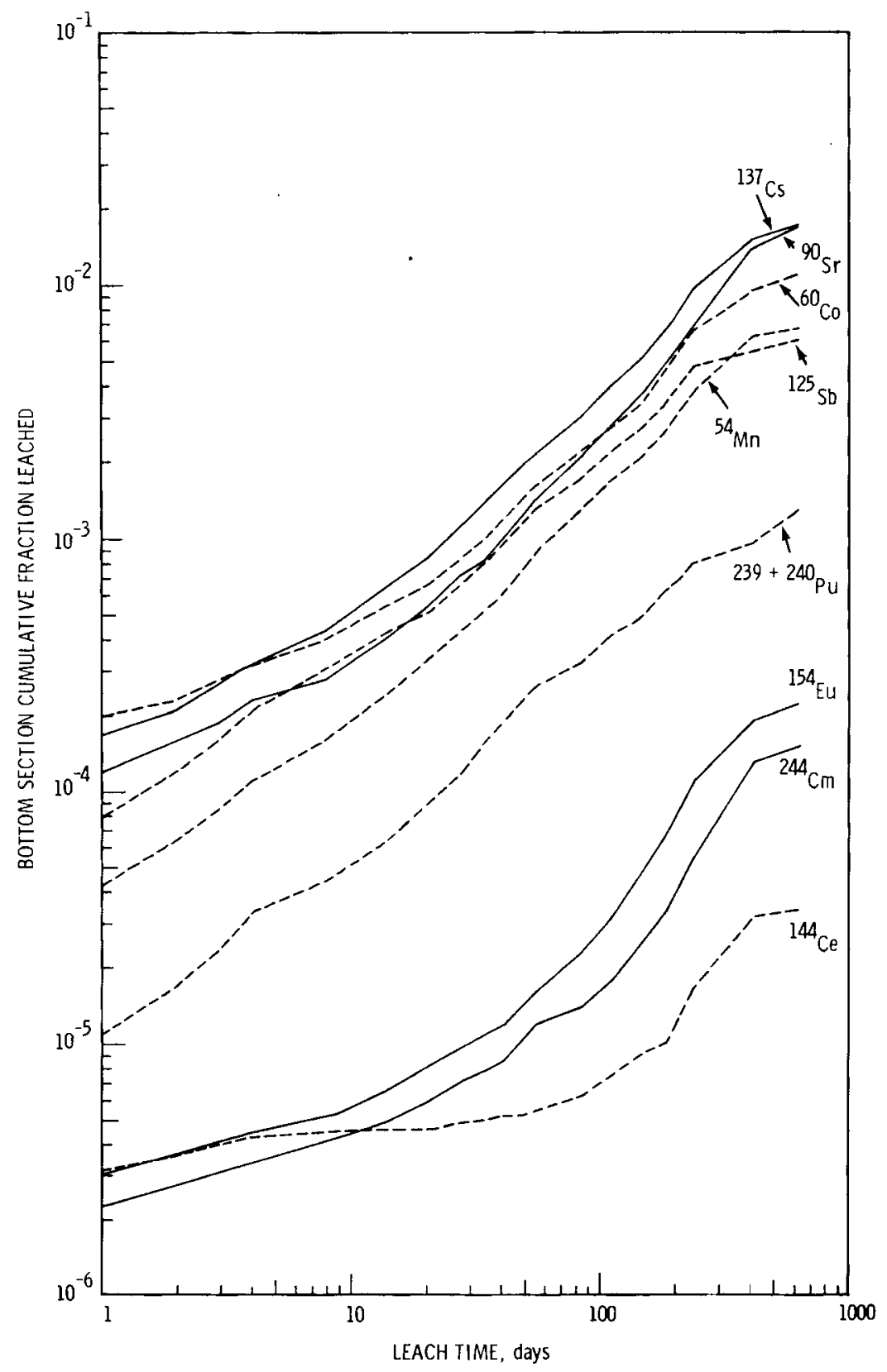

FIGURE 11. Cumulative Fractions Leached as a Function of Time, Bottom Section 
TABLE 8. Element Release Fractions After 639 Days

\begin{tabular}{|c|c|c|}
\hline Element & $\begin{array}{l}\text { Fraction Released } \\
\text { at } 639 \text { Days }(a)\end{array}$ & Source \\
\hline Cs & $2.8 \times 10^{-2}$ & Fission product \\
\hline $\mathrm{Sr}$ & $2.0 \times 10^{-2}$ & Fission product \\
\hline Co & $1.6 \times 10^{-2}$ & Activation product \\
\hline $\mathrm{Sb}$ & $1.1 \times 10^{-2}$ & Fission product \\
\hline$M n$ & $7.7 \times 10^{-3}$ & Activation product \\
\hline $\mathrm{Pu}$ & $2.3 \times 10^{-3}$ & Actinide \\
\hline $\mathrm{Eu}$ & $2.6 \times 10^{-4}$ & Fission product \\
\hline $\mathrm{Cm}$ & $1.9 \times 10^{-4}$ & Actinide \\
\hline $\mathrm{Ce}$ & $4.8 \times 10^{-5}$ & Fission product \\
\hline
\end{tabular}

(a) Averaged over the four glass sections

Changes in the leach rate curves due to sampling frequency are reflected both in the graphs of leach rate (Figures 5-7) and in the graphs of cumulative fractions leached (Figures 10 and 11 ). The plots of cumulative fractions leached show that several mechanisms have controlled release behavior. Fractional release can be expressed using Fick's Law as: ${ }^{(6)}$

$$
F_{r}=b T^{m}
$$

or:

$$
\log F_{r}=m \log T+\log b=m \log T+B
$$

where $F_{r}=$ Isotope fraction released

$T=$ Time

$B=$ Intercept

$\mathrm{m}=$ Slope of the line.

The slope is indicative of the type of leaching mechanism. A slope of 0.5 indicates a diffusion controlled release; a slope of 1.0 indicates 
a silicate lattice alteration mechanism, commonly referred to as corrosion. $(6,8,9)$ The diffusion release mechanism is characterized by surface adsorption, ion exchange, and migration. Chemical attack or alteration of the silicate lattice is characterized by hydroxyl attack on silicon or hydrogen attack on bridging oxygens. (10)

To illustrate the effect of sampling frequency, slopes were calculated from sections of the graphs of cumulative fractions leached for the initial leaching period ( 1 to 10 days), periods of constant sampling frequency ( 10 to 56 days and 100 to 240 days), and periods following a change in sampling frequency. These are tabulated in Table 9. The change from daily to weekly sampling seems to have had very little effect on the slope and, for this reason, was included in the first 10 days of the leaching period. Extensive leaching time on a monthly frequency shows a definite shift to a lattice alteration mechanism for a11 elements except plutonium, which appears to remain stable at a point between the two mechanisms. This behavior is most likely to be a combination of diffusion and corrosion release as discussed by Douglas (9) and Diebold. (10) The change from weekly to monthly sampling times appears to have had little effect, except possibly for extending the time needed to approach a slope of 1 . The effect of the change in sampling frequency from monthly to semi-annually is dramatic. The apparent mechanism shifted from lattice alteration to diffusion release; this shift illustrates the important role of dissolved species in lowering the leach rate. These results are consistent with the work of El-Shamy (11) and Paul. (12)

From the curves of cumulative fraction release, the general trend over the total testing time shows two different slopes for each element. All of the elements have a slope less than 0.50 at the beginning of the leach test, indicating a type of diffusion controlled release. After a period of time this slope gradually approaches a value of 1 , indicating a combination of release mechanisms. This result is consistent with discussion of release mechanisms in the 1 iterature. $(6,8,10)$ This leaching study is being continued to observe the longer term leaching behavior. Table 10 summarizes these results by element for the top and bottom sections of high-level waste glass. 
TABLE 9. Slopes Calculated from Curves of Cumulative Fractions Leached

\begin{tabular}{|c|c|c|c|c|c|c|c|c|c|c|c|}
\hline & & Time $=$ & 0 days & Time $=$ & 6 days & Time $=5$ & 00 days & Time $=$ & 40 days & Time $=2$ & 39 days \\
\hline & Isotope & Bottom & Top & Bottom & Top & Bottom & Top & Bottom & Top & Bottom & Top \\
\hline & ${ }^{54} \mathrm{Mn}$ & 0.65 & 0.30 & 0.90 & 0.87 & 0.92 & 0.94 & 1.00 & 1.25 & 0.59 & 0.38 \\
\hline & ${ }^{60} \mathrm{Co}$ & 0.35 & 0.38 & 0.68 & 0.81 & 0.76 & 0.89 & 1.03 & 1.06 & 0.64 & 0.53 \\
\hline & ${ }^{90} \mathrm{Sr}$ & 0.43 & 0.25 & 0.87 & 0.64 & 0.97 & 0.81 & 1.13 & 1.06 & 0.93 & 0.74 \\
\hline & ${ }^{125} \mathrm{Sb}$ & 0.63 & 0.48 & 0.77 & 0.55 & 0.73 & .78 & 0.97 & 0.84 & 0.24 & 0.44 \\
\hline$\hat{a}$ & ${ }^{137} \mathrm{Cs}$ & 0.48 & 0.43 & 0.84 & 0.92 & 0.89 & 1.11 & 1.06 & 1.19 & 0.60 & 0.56 \\
\hline & ${ }^{144} \mathrm{Ce}$ & 0.15 & 0.11 & 0.11 & 0.13 & 0.43 & 0.16 & 0.94 & 0.56 & 0.71 & 0.80 \\
\hline & ${ }^{154} \mathrm{Eu}$ & 0.26 & 0.28 & 0.61 & 0.45 & 0.91 & 0.43 & 1.50 & 1.03 & 0.71 & 0.56 \\
\hline & ${ }^{239} \mathrm{Pu}$ & 0.66 & 0.24 & 0.94 & 0.84 & 0.64 & 0.66 & 0.84 & 0.72 & 0.51 & 0.19 \\
\hline & ${ }^{244} \mathrm{~cm}$ & 0.29 & $\underline{0.35}$ & $\underline{0.65}$ & 0.48 & 0.51 & $\underline{0.25}$ & 1.38 & $\underline{0.81}$ & 1.02 & 0.47 \\
\hline & Ave. & 0.43 & 0.31 & 0.71 & 0.63 & 0.75 & 0.67 & 1.09 & 0.95 & 0.66 & 0.52 \\
\hline & Ave. & & & & & & & & & & \\
\hline
\end{tabular}


TABLE 10. Slopes of Initial and Long-Term Rel ease Mechanisms

\begin{tabular}{|c|c|c|}
\hline Elements & $\begin{array}{c}\text { Initial Slope/ } \\
\text { Time Period, Days }\end{array}$ & $\begin{array}{l}\text { Long-Term Slope/ } \\
\text { Time Period, Days }\end{array}$ \\
\hline $\mathrm{Mn}$ & $0.31 / 1-8$ & $0.84 / 8-639$ \\
\hline Co & $0.33 / 1-8$ & $0.81 / 8-639$ \\
\hline $\mathrm{Sr}$ & $0.24 / 1-8$ & $0.76 / 8-639$ \\
\hline $\mathrm{Sb}$ & $0.47 / 1-30$ & $0.67 / 30-639$ \\
\hline Cs & $0.40 / 1-8$ & $0.91 / 8-639$ \\
\hline $\mathrm{Ce}$ & $0.12 / 1-100$ & $0.70 / 100-639$ \\
\hline $\mathrm{Eu}$ & $0.31 / 1-20$ & $0.66 / 20-639$ \\
\hline $\mathrm{Pu}$ & $0.21 / 1-8$ & $0.64 / 8-639$ \\
\hline $\mathrm{Cm}$ & $0.39 / 1-150$ & $0.72 / 150-639$ \\
\hline
\end{tabular}

The scope of this work was not intended to study and analyze leaching mechanisms beyond this point, but the data do show that the mechanism is dependent on time, sampling frequency, and type of element. Also, over the long testing period, two different mechanisms account for the release of material. More work is needed to increase our understanding of these high-level waste glass - solution interactions. 
$\because$ 


\section{REFERENCES}

1. M. J. Bel1, ORIGEN - the ORNL Isotope Generation and Depletion Code. ORNL-4628, Oak Ridge National Laboratory, Oak Ridge, TN 37830, May 1973.

2. J. E. Mendel, W. A. Ross, F. P. Roberts, Y. B. Katayama, J. H. Westsik, Jr., R. P. Turcotte, J. W. Wald and D. J. Bradley, Annual Report on the Characteristics of High-Level Waste Glasses. BNWL-2252, Battelle, Pacific Northwest Laboratories, Richland, WA 99352, June 1977.

3. W. A. Ross, D. J. Bradley, R. L. Bunnel1, W. J. Gray, Y. B. Katayama, G. B. Mellinger, J. E. Mende 1, F. P. Roberts, R. P. Turcotte, J.W. Wald, W. E. Weber and J. H. Westsik, Jr. Annual Report on the Characteristics of High-Level Waste Glasses. BNWL-2625, Battelle, Pacific Northwest Laboratories, Richland, WA 99352, June 1978.

4. E. D. Hespe, ed., "Leach Testing of Immobilized Radioactive Waste Solids, A Proposal for a Standard Method." Atomic Energy Review 9:1, 1971.

5. P. Arthur and 0. M. Smith, "Semi-Micro Qualitative Analysis," International Chemistry Series. McGraw Hi11, New York, NY, 1942.

6. Y. B. Katayama, Leaching of Irradiated LWR Fuel Pellets in Deionized and Typical Ground Water. BNWL-2057, Battelle, Pacific Northwest Laboratories, Richland, WA 99352, July 1976.

7. J. E. Mende 1, W. A. Ross, F. P. Roberts, R. P. Turcotte, Y. B. Yatayama and J. H. Westsik, Jr., "Thermal and Radiation Effects on Borosilicate Waste Glasses." IAEA Symposium on Management of Radioactive Waste from the Nuclear Fuel Cycle, IAEA-SM-207/100, 2:49, Vienna, 1976.

8. L. L. Hench, "Leaching of Glass." Workshop on Ceramic and Glass Radioactive Waste Forms, Germantown, MD, January 1977.

9. R. W. Douglas and T. M. M. El-Shamy, "Reactions of Glasses with Aqueous Solutions." Journal of American Ceramic Society, 50(1):1-8, January 21, 1967.

10. F. E. Diebold, Discussions of Glass - Water Interactions. ARH-2905, September 15, $1 \overline{973 .}$

11. T. M. M. El-Shamy, PhD. Thesis, University of Sheffield, Sheffield, UK, 1966.

12. A. Pau1, Chemical Durability of Glasses, A Thermodynamic Approach. University of Sheffield, Sheffield, UK, 1977. 
$\because$ 


\section{ACKNOWLEDGMENTS}

The author appreciates the help of $\mathrm{Y}$. B. Katayama on the glass preparation;

J. H. Westsik, J. E. Mendel, and R. P. Turcotte on the text; J. C. Nelson,

C. E. Bigelow, and J. R. Crockett for performing the hot cell work; and

L. R. Jaech for editing the report. 


\section{APPENDIX A}

ISOTOPIC COMPOSITION OF SPENT

REACTOR FUEL AS CALCULATED BY

AN ORIGEN CODE COMPUTER RUN 
APPENDIX A

\section{ISOTOPIC COMPOSITION OF SPENT REACTOR FUEL AS CALCULATED BY AN ORIGEN ${ }^{(2)}$ CODE COMPUTER RUN $(\mathrm{a})$}

\begin{tabular}{|c|c|c|c|}
\hline Isotope & $\operatorname{Grams}(b)$ & Isotope & $\operatorname{Grams}^{(b)}$ \\
\hline${ }^{74} \mathrm{Ge}$ & $1.36 \mathrm{E}-04^{(c)}$ & ${ }^{95}{ }_{\text {MO }}$ & $1.99 \mathrm{E}+00$ \\
\hline${ }^{75}$ As & $2.19 \mathrm{E}-04$ & ${ }^{96} \mathrm{Zr}$ & $2.20 \mathrm{E}+00$ \\
\hline${ }^{76} \mathrm{Ge}$ & $7.55 E-04$ & ${ }^{96}{ }_{\mathrm{Mo}}$ & $1.53 \mathrm{E}-01$ \\
\hline${ }^{77} \mathrm{Se}$ & $2.39 E-03$ & ${ }^{97}$ Mo & $2.18 \mathrm{E}+00$ \\
\hline${ }^{78} \mathrm{Se}$ & $6.54 \mathrm{E}-03$ & ${ }^{98}$ Мо & $2.21 E+00$ \\
\hline${ }^{79} \mathrm{Se}$ & 1.53E-02 & ${ }^{99} \mathrm{TC}$ & $2.14 \mathrm{E}+00$ \\
\hline${ }^{80} \mathrm{Se}$ & $2.68 \mathrm{E}-02$ & ${ }^{100}$ Mo & $2.51 E+00$ \\
\hline${ }^{81} \mathrm{Br}$ & $4.00 E-02$ & ${ }^{100} \mathrm{Ru}$ & $1.92 \mathrm{E}-01$ \\
\hline${ }^{82} \mathrm{Se}$ & $8.84 \mathrm{E}-02$ & ${ }^{101} \mathrm{Ru}$ & $1.97 E+00$ \\
\hline${ }^{85} \mathrm{Rb}$ & $2.73 \mathrm{E}-01$ & ${ }^{102} \mathrm{Ru}$ & $1.96 \mathrm{E}+00$ \\
\hline${ }^{86} \mathrm{Sr}$ & $4.45 \mathrm{E}-04$ & ${ }^{103} \mathrm{Rh}$ & 8.01E-01 \\
\hline${ }^{87} \mathrm{Rb}$ & $6.63 E-01$ & ${ }^{104} \mathrm{Ru}$ & $1.29 \mathrm{E}+00$ \\
\hline${ }^{88} \mathrm{Sr}$ & $9.67 \mathrm{E}-01$ & ${ }^{104} \mathrm{Pd}$ & $7.64 \mathrm{E}-01$ \\
\hline $89_{Y}$ & 1. $30 E+00$ & ${ }^{105} \mathrm{Pd}$ & $6.98 \mathrm{E}-01$ \\
\hline${ }^{90} \mathrm{Sr}$ & $1.43 E+00$ & ${ }^{106} \mathrm{Ru}$ & $8.64 \mathrm{E}-02$ \\
\hline $90_{Y}$ & $3.70 \mathrm{E}-04$ & ${ }^{106} \mathrm{Pd}$ & $8.83 E-01$ \\
\hline${ }^{90} \mathrm{Zr}$ & $1.60 \mathrm{E}-01$ & ${ }^{107} \mathrm{Pd}$ & $4.95 \mathrm{E}-01$ \\
\hline${ }^{91} \mathrm{Zr}$ & $1.67 E+00$ & ${ }^{108} \mathrm{Pd}$ & $3.27 \mathrm{E}-01$ \\
\hline${ }^{92} \mathrm{Zr}$ & $1.81 E+00$ & ${ }^{109} \mathrm{Ag}$ & $1.14 \mathrm{E}-01$ \\
\hline${ }^{93} \mathrm{Zr}$ & $1.97 \mathrm{E}+00$ & $110_{\mathrm{Pd}}$ & $7.14 \mathrm{E}-02$ \\
\hline${ }^{94} \mathrm{Zr}$ & $2.13 E+00$ & $110 \mathrm{~m}_{\mathrm{Ag}}$ & $3.04 \mathrm{E}-04$ \\
\hline${ }^{95} \mathrm{Zr}$ & $1.59 \mathrm{E}-04$ & ${ }^{110} \mathrm{Cd}$ & $1.05 \mathrm{E}-01$ \\
\hline${ }^{95} \mathrm{Nb}$ & $1.84 E-04$ & ${ }^{111} \mathrm{Cd}$ & $3.78 \mathrm{E}-02$ \\
\hline
\end{tabular}

(a) Noble gases are not included. Only isotopes present in quantities greater than $10^{-4} \mathrm{~g}$ are given.

(b) Based on one fuel rod of $1564 \mathrm{~g}$.

(c) $1.36 \mathrm{E}-04=1.36 \times 10^{-4}$. 


\begin{tabular}{|c|c|c|c|}
\hline Isotope & $\operatorname{Grams}(a)$ & Isotope & $\operatorname{Grams}^{(a)}$ \\
\hline${ }^{112} \mathrm{Cd}$ & $2.07 E-02^{(b)}$ & $134 \mathrm{Ba}$ & $5.50 \mathrm{E}-01$ \\
\hline $113 m_{C d}$ & $1.24 \mathrm{E}-04$ & ${ }^{135} \mathrm{Cs}$ & $1.04 E+00$ \\
\hline $113 \mathrm{Cd}$ & $3.87 E-04$ & $135_{\mathrm{Ba}}$ & $2.15 \mathrm{E}-04$ \\
\hline${ }^{114} \mathrm{Cd}$ & $2.94 \mathrm{E}-02$ & ${ }^{135} \mathrm{Ba}$ & $2.15 \mathrm{E}-04$ \\
\hline $115_{\text {In }}$ & $2.19 E-03$ & ${ }^{136} \mathrm{Ba}$ & $9.14 \mathrm{E}-02$ \\
\hline${ }^{11} 5_{S n}$ & $4.56 E-04$ & ${ }^{137} \mathrm{Cs}$ & $3.02 E+00$ \\
\hline${ }^{116} \mathrm{Cd}$ & $8.82 E-03$ & ${ }^{137} \mathrm{Ba}$ & $2.96 \mathrm{E}-01$ \\
\hline $116_{\mathrm{Sn}}$ & $7.15 \mathrm{E}-03$ & ${ }^{138} \mathrm{Ba}$ & $3.14 E+00$ \\
\hline${ }^{117} \mathrm{Sn}$ & $9.15 \mathrm{E}-03$ & 13 & $3.32 E+00$ \\
\hline $118_{S n}$ & $9.38 \mathrm{E}-03$ & 140 & $3.40 E+00$ \\
\hline $119_{S n}$ & $9.72 E-03$ & & $3.16 \mathrm{E}+00$ \\
\hline${ }^{120} \mathrm{Sn}$ & $1.02 E-02$ & ${ }^{142} \mathrm{Ce}$ & $3.10 E+00$ \\
\hline${ }^{121} \mathrm{Sb}$ & $1.05 \mathrm{E}-02$ & ${ }^{142} \mathrm{Nd}$ & $7.43 E-02$ \\
\hline${ }^{122} \mathrm{Sn}$ & $1.21 \mathrm{E}-02$ & $143 \mathrm{Nd}$ & $1.95 \mathrm{E}+00$ \\
\hline${ }^{122} \mathrm{~T}$ & $9.77 \mathrm{E}-04$ & & $1.36 \mathrm{E}-01$ \\
\hline${ }^{123} \mathrm{Sb}$ & $1.27 \mathrm{E}-02$ & ${ }^{144} \mathrm{Nd}$ & $3.63 E+00$ \\
\hline $124_{s}$ & $1.78 \mathrm{E}-02$ & & $1.79 E+00$ \\
\hline${ }^{124} 4_{T}$ & $4.99 E-04$ & $146_{\mathrm{Nd}}$ & $1.91 E+00$ \\
\hline${ }^{125} \mathrm{Sb}$ & $1.07 E-02$ & ${ }^{147} \mathrm{Pm}$ & $1.35 E-01$ \\
\hline $125 \mathrm{~m} \mathrm{Te}$ & $2.61 \mathrm{E}-04$ & ${ }^{147} \mathrm{Sm}$ & 2.32E-01 \\
\hline${ }^{125} \mathrm{Te}$ & $1.46 \mathrm{E}-02$ & $148 \mathrm{Nd}$ & $9.66 \mathrm{E}-01$ \\
\hline${ }^{126} \mathrm{Sn}$ & $4.43 E-02$ & ${ }^{148} \mathrm{Sm}$ & $5.48 E-01$ \\
\hline${ }^{126} \mathrm{Te}$ & $1.63 \mathrm{E}-04$ & ${ }^{149} \mathrm{Sm}$ & $1.38 \mathrm{E}-02$ \\
\hline 127 I & $9.22 \mathrm{E}-02$ & $150 \mathrm{Nd}$ & $4.44 E-01$ \\
\hline${ }^{128} \mathrm{Te}$ & $3.18 \mathrm{E}-01$ & & $9.51 \mathrm{E}-01$ \\
\hline $129 \mathrm{~m} \mathrm{Te}$ & $1.22 \mathrm{E}-08$ & ${ }^{151} \mathrm{Sm}$ & $9.92 E-02$ \\
\hline${ }^{129} \mathrm{I}$ & $5.64 \mathrm{E}-01$ & & $1.47 \mathrm{E}-03$ \\
\hline${ }^{130} \mathrm{Te}$ & $1.08 \mathrm{E}+00$ & ${ }^{152} \mathrm{Sm}$ & $2.08 \mathrm{E}-01$ \\
\hline${ }^{133} \mathrm{Cs}$ & $2.42 \mathrm{E}+00$ & ${ }^{152} \mathrm{Eu}$ & $1.50 \mathrm{E}-04$ \\
\hline${ }^{134} \mathrm{Cs}$ & $3.11 E-01$ & ${ }^{152} \mathrm{Gd}$ & $5.50 E-04$ \\
\hline
\end{tabular}

(a) Based on one fuel rod of $1564 \mathrm{~g}$.

(b) $2.07 \mathrm{E}-02=2.07 \times 10-2$ 


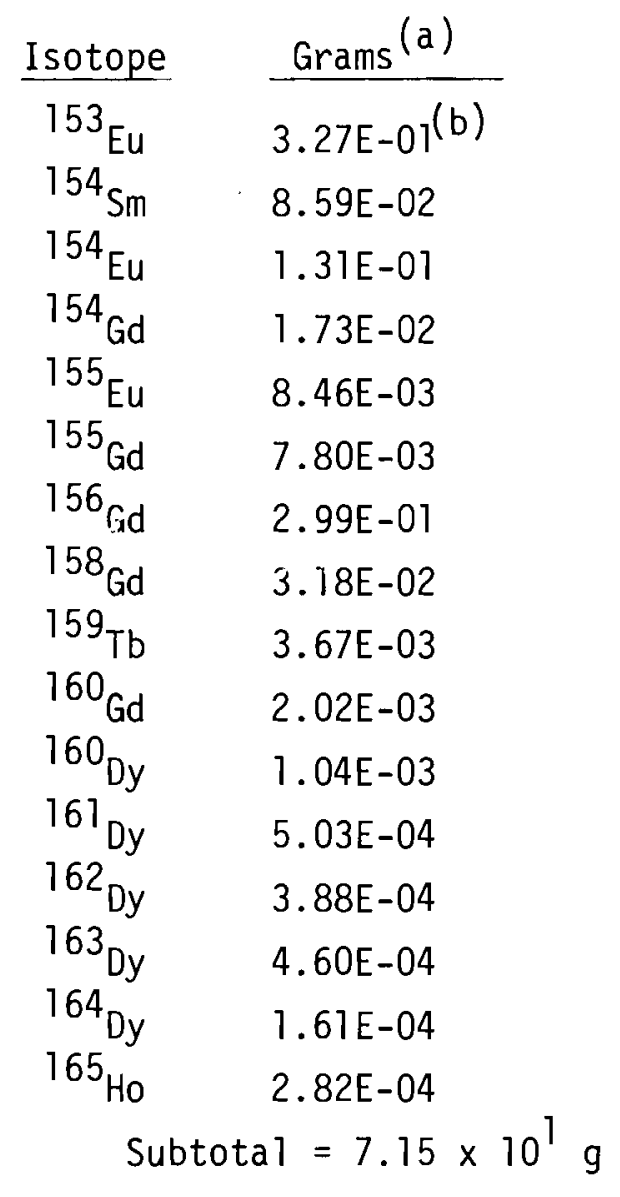

\begin{tabular}{|c|c|}
\hline Isotope & $\operatorname{Grams}^{(a)}$ \\
\hline $234 U$ & $1.71 \mathrm{E}-03$ \\
\hline $235 \mathrm{U}$ & $7.94 \mathrm{E}-02$ \\
\hline $236_{U}$ & $6.52 E-02$ \\
\hline $238 u$ & $7.13 E+00$ \\
\hline $237 \mathrm{~Np}$ & $1.63 E+00$ \\
\hline${ }^{238} \mathrm{Pu}$ & $3.45 E-03$ \\
\hline${ }^{239} \mathrm{Pu}$ & $4.22 E-02$ \\
\hline${ }^{240} \mathrm{Pu}$ & $1.82 \mathrm{E}-02$ \\
\hline${ }^{241} \mathrm{Pu}$ & $8.92 E-03$ \\
\hline${ }^{242} \mathrm{Pu}$ & $4.03 E-03$ \\
\hline${ }^{241} \mathrm{Am}$ & $2.03 E-01$ \\
\hline $242 m_{A m}$ & $2.37 E-03$ \\
\hline${ }^{243} \mathrm{Am}$ & $2.72 \mathrm{E}-01$ \\
\hline${ }^{242} \mathrm{Cm}$ & $1.96 \mathrm{E}-03$ \\
\hline${ }^{243} \mathrm{Cm}$ & $2.34 \mathrm{E}-04$ \\
\hline${ }^{244} \mathrm{Cm}$ & $1.07 E-01$ \\
\hline${ }^{245} \mathrm{Cm}$ & $8.75 E-03$ \\
\hline${ }^{246} \mathrm{Cm}$ & $1.29 \mathrm{E}-03$ \\
\hline
\end{tabular}

(a) Based on one fuel rod of $1564 \mathrm{~g}$.

(b) $3.27 \mathrm{E}-01=3.27 \times 10^{-1}$ 
:

$\because$ 
APPENDIX B

LEACHING DATA 


\section{APPENDIX B}

\begin{tabular}{|c|c|c|}
\hline \multirow[b]{2}{*}{ Series \# } & \multicolumn{2}{|c|}{${ }^{54} \mathrm{Mn}$-Bottom Section } \\
\hline & $\begin{array}{l}\text { Leach Rate } \\
\mathrm{g} / \mathrm{cm}^{2} \text {-day }\end{array}$ & $\begin{array}{c}\text { Cumulative } \\
\text { Fraction Leached } \\
\end{array}$ \\
\hline 1 & $2.7 \times 10^{-5}$ & $4.3 \times 10^{-5}$ \\
\hline 2 & $1.4 \times 10^{-6}$ & $6.5 \times 10^{-5}$ \\
\hline 3 & $1.3 \times 10^{-6}$ & $8.6 \times 10^{-5}$ \\
\hline 4 & $1.7 \times 10^{-6}$ & $1.1 \times 10^{-4}$ \\
\hline 5 & $6.9 \times 10^{-6}$ & $1.6 \times 10^{-4}$ \\
\hline 6 & $8.5 \times 10^{-6}$ & $2.4 \times 10^{-4}$ \\
\hline 7 & $8.9 \times 10^{-6}$ & $3.4 \times 10^{-4}$ \\
\hline 8 & $9.6 \times 10^{-6}$ & $4.4 \times 10^{-4}$ \\
\hline 9 & $7.5 \times 10^{-6}$ & $5.2 \times 10^{-4}$ \\
\hline 10 & $1.0 \times 10^{-5}$ & $6.3 \times 10^{-4}$ \\
\hline 11 & $1.1 \times 10^{-5}$ & $7.5 \times 10^{-4}$ \\
\hline 12 & $1.2 \times 10^{-5}$ & $8.8 \times 10^{-4}$ \\
\hline 13 & $8.3 \times 10^{-6}$ & $1.3 \times 10^{-3}$ \\
\hline 14 & $8.3 \times 10^{-6}$ & $1.7 \times 10^{-3}$ \\
\hline 15 & $8.4 \times 10^{-6}$ & $2.1 \times 10^{-3}$ \\
\hline 16 & $9.0 \times 10^{-6}$ & $2.6 \times 10^{-3}$ \\
\hline 17 & $1.2 \times 10^{-5}$ & $3.1 \times 10^{-3}$ \\
\hline 18 & $1.6 \times 10^{-5}$ & $3.8 \times 10^{-3}$ \\
\hline 19 & $8.2 \times 10^{-6}$ & $6.3 \times 10^{-3}$ \\
\hline 20 & $1.1 \times 10^{-6}$ & $6.7 \times 10^{-3}$ \\
\hline
\end{tabular}




\section{${ }^{54}$ Mn-Lower-Middle Section}

\begin{tabular}{ccc} 
Series \# & $\begin{array}{c}\text { Leach Rate } \\
\mathrm{g} / \mathrm{cm}^{2}-\text { day }\end{array}$ & $\begin{array}{c}\text { Cumulative } \\
\text { Fraction Leached }\end{array}$ \\
\hline 1 & $3.0 \times 10^{-5}$ & $6.8 \times 10^{-5}$ \\
2 & $1.2 \times 10^{-5}$ & $9.5 \times 10^{-5}$ \\
3 & $7.4 \times 10^{-6}$ & $1.1 \times 10^{-4}$ \\
4 & $1.2 \times 10^{-5}$ & $1.4 \times 10^{-4}$ \\
5 & $5.6 \times 10^{-6}$ & $1.9 \times 10^{-4}$ \\
6 & $7.1 \times 10^{-6}$ & $2.9 \times 10^{-4}$ \\
7 & $5.9 \times 10^{-6}$ & $3.8 \times 10^{-4}$ \\
8 & $6.8 \times 10^{-6}$ & $4.9 \times 10^{-4}$ \\
9 & $5.0 \times 10^{-6}$ & $5.7 \times 10^{-4}$ \\
10 & $7.0 \times 10^{-6}$ & $6.8 \times 10^{-4}$ \\
* & $6.2 \times 10^{-6}$ & $7.8 \times 10^{-4}$ \\
11 & $1.6 \times 10^{-5}$ & $1.0 \times 10^{-3}$ \\
12 & $6.4 \times 10^{-6}$ & $1.5 \times 10^{-3}$ \\
13 & $5.2 \times 10^{-6}$ & $1.8 \times 10^{-3}$ \\
14 & $6.8 \times 10^{-6}$ & $2.3 \times 10^{-3}$ \\
15 & $9.7 \times 10^{-6}$ & $3.1 \times 10^{-3}$ \\
16 & $1.2 \times 10^{-5}$ & $3.9 \times 10^{-3}$ \\
17 & $1.5 \times 10^{-5}$ & $4.8 \times 10^{-3}$ \\
18 & $3.0 \times 10^{-6}$ & $6.1 \times 10^{-3}$ \\
19 & $1.7 \times 10^{-6}$ & $7.0 \times 10^{-3}$ \\
20 & &
\end{tabular}




\section{${ }^{54}$ Mn-Upper-Middle Section}

$\begin{array}{ccc}\text { Series \# } & \begin{array}{c}\text { Leach Rate } \\ \mathrm{g} / \mathrm{cm}^{2} \text {-day }\end{array} & \begin{array}{c}\text { Cumulative } \\ \text { Fraction Leached }\end{array} \\ 1 & \begin{array}{c}2.2 \times 10^{-5} \\ 2\end{array} & \begin{array}{l}1.1 \times 10^{-5} \\ 4.6 \times 10^{-5}\end{array} \\ 3 & 1.0 \times 10^{-5} & 6.8 \times 10^{-5} \\ 4 & 1.4 \times 10^{-5} & 8.9 \times 10^{-5} \\ 5 & 7.2 \times 10^{-6} & 1.2 \times 10^{-4} \\ 6 & 5.0 \times 10^{-6} & 1.8 \times 10^{-4} \\ 7 & 7.0 \times 10^{-6} & 2.4 \times 10^{-4} \\ 8 & 7.8 \times 10^{-6} & 3.4 \times 10^{-4} \\ 9 & 6.4 \times 10^{-6} & 4.6 \times 10^{-4} \\ 10 & 8.0 \times 10^{-6} & 5.5 \times 10^{-4} \\ 11 & 5.2 \times 10^{-6} & 6.7 \times 10^{-4} \\ 12 & 7.0 \times 10^{-6} & 7.5 \times 10^{-4} \\ 13 & 3.1 \times 10^{-6} & 8.5 \times 10^{-4} \\ 14 & 5.1 \times 10^{-6} & 1.0 \times 10^{-3} \\ 15 & 4.5 \times 10^{-6} & 1.4 \times 10^{-3} \\ 16 & 8.2 \times 10^{-6} & 1.7 \times 10^{-3} \\ 17 & 1.0 \times 10^{-5} & 2.2 \times 10^{-3} \\ 18 & 9.8 \times 10^{-6} & 2.9 \times 10^{-3} \\ 19 & 2.3 \times 10^{-6} & 3.4 \times 10^{-3} \\ 20 & 1.0 \times 10^{-6} & 4.3 \times 10^{-3} \\ & & 5.1 \times 10^{-3}\end{array}$




\section{${ }^{54} \mathrm{Mn}$-Top Section}

$\underline{\text { Series \# }} \quad \begin{array}{r}\text { Leach Rate } \\ \mathrm{g} / \mathrm{cm}^{2} \text {-day }\end{array} \quad \begin{gathered}\text { Cumulative } \\ \text { Fraction Leached }\end{gathered}$

$\begin{array}{lll}1 & 6.5 \times 10^{-5} & 1.7 \times 10^{-4} \\ 2 & 7.9 \times 10^{-6} & 1.9 \times 10^{-4} \\ 3 & 7.5 \times 10^{-6} & 2.1 \times 10^{-4} \\ 4 & 8.1 \times 10^{-6} & 2.4 \times 10^{-4} \\ 5 & 5.7 \times 10^{-6} & 3.0 \times 10^{-4} \\ 6 & 6.4 \times 10^{-6} & 4.0 \times 10^{-4} \\ 7 & 8.0 \times 10^{-6} & 5.5 \times 10^{-4} \\ 8 & 8.8 \times 10^{-6} & 7.1 \times 10^{-4} \\ 9 & 7.5 \times 10^{-6} & 8.5 \times 10^{-4} \\ 10 & 1.1 \times 10^{-5} & 1.1 \times 10^{-3} \\ \text { * } & 8.8 \times 10^{-6} & 1.2 \times 10^{-3} \\ 11 & 1.4 \times 10^{-5} & 1.5 \times 10^{-3} \\ 12 & 9.3 \times 10^{-6} & 2.2 \times 10^{-3} \\ 13 & 1.0 \times 10^{-5} & 3.0 \times 10^{-3} \\ 14 & 1.3 \times 10^{-5} & 4.2 \times 10^{-3} \\ 15 & 1.5 \times 10^{-5} & 5.5 \times 10^{-3} \\ 16 & 1.6 \times 10^{-5} & 6.7 \times 10^{-3} \\ 17 & 2.0 \times 10^{-5} & 8.2 \times 10^{-3} \\ 18 & 7.6 \times 10^{-6} & 1.2 \times 10^{-2} \\ 19 & 1.1 \times 10^{-6} & 1.2 \times 10^{-2} \\ 20 & & \end{array}$




\begin{tabular}{|c|c|c|}
\hline \multirow[b]{2}{*}{ Series \# } & \multicolumn{2}{|c|}{${ }^{60}$ Co-Bottom Section } \\
\hline & $\begin{array}{r}\text { Leach Rate } \\
\mathrm{g} / \mathrm{cm}^{2}-\text { day } \\
\end{array}$ & $\begin{array}{c}\text { Cumulative } \\
\text { Fraction Leached }\end{array}$ \\
\hline 1 & $1.2 \times 10^{-4}$ & $2.0 \times 10^{-4}$ \\
\hline 2 & $2.3 \times 10^{-5}$ & $2.3 \times 10^{-4}$ \\
\hline 3 & $2.7 \times 10^{-5}$ & $2.8 \times 10^{-4}$ \\
\hline 4 & $2.6 \times 10^{-5}$ & $3.2 \times 10^{-4}$ \\
\hline 5 & $1.4 \times 10^{-5}$ & $4.0 \times 10^{-4}$ \\
\hline 6 & $1.4 \times 10^{-5}$ & $5.4 \times 10^{-4}$ \\
\hline 7 & $1.1 \times 10^{-5}$ & $6.6 \times 10^{-4}$ \\
\hline 8 & $1.6 \times 10^{-5}$ & $8.4 \times 10^{-4}$ \\
\hline 9 & $1.5 \times 10^{-5}$ & $1.0 \times 10^{-3}$ \\
\hline 10 & $1.6 \times 10^{-5}$ & $1.2 \times 10^{-3}$ \\
\hline 11 & $1.7 \times 10^{-5}$ & $1.4 \times 10^{-3}$ \\
\hline 12 & $1.7 \times 10^{-5}$ & $1.6 \times 10^{-3}$ \\
\hline 13 & $1.3 \times 10^{-5}$ & $2.2 \times 10^{-3}$ \\
\hline 14 & $1.2 \times 10^{-5}$ & $2.7 \times 10^{-3}$ \\
\hline 15 & $1.3 \times 10^{-5}$ & $3.4 \times 10^{-3}$ \\
\hline 16 & $1.7 \times 10^{-5}$ & $4.3 \times 10^{-3}$ \\
\hline 17 & $2.5 \times 10^{-5}$ & $5.4 \times 10^{-3}$ \\
\hline 18 & $2.3 \times 10^{-5}$ & $6.5 \times 10^{-3}$ \\
\hline 19 & $1.1 \times 10^{-5}$ & $9.5 \times 10^{-3}$ \\
\hline 20 & $3.2 \times 10^{-6}$ & $1.1 \times 10^{-2}$ \\
\hline
\end{tabular}




\section{${ }^{60}$ Co-Lower-Middle Section}

Series \# $\quad \begin{array}{cc}\text { Leach Rate } \\ \mathrm{g} / \mathrm{cm}^{2} \text {-day }\end{array} \quad \begin{gathered}\text { Cumulative } \\ \text { Fraction Leached }\end{gathered}$

$\begin{array}{lll}1 & 7.2 \times 10^{-5} & 1.6 \times 10^{-4} \\ 2 & 1.3 \times 10^{-5} & 1.9 \times 10^{-4} \\ 3 & 1.5 \times 10^{-5} & 2.3 \times 10^{-4} \\ 4 & 1.7 \times 10^{-5} & 2.7 \times 10^{-4} \\ 5 & 1.3 \times 10^{-5} & 3.8 \times 10^{-4} \\ 6 & 1.2 \times 10^{-5} & 5.5 \times 10^{-4} \\ 7 & 9.9 \times 10^{-6} & 7.0 \times 10^{-4} \\ 8 & 1.4 \times 10^{-5} & 9.2 \times 10^{-4} \\ 9 & 1.3 \times 10^{-5} & 1.1 \times 10^{-3} \\ 10 & 9.8 \times 10^{-6} & 1.3 \times 10^{-3} \\ \text { * } & 1.2 \times 10^{-5} & 1.5 \times 10^{-3} \\ 11 & 1.9 \times 10^{-5} & 1.8 \times 10^{-3} \\ 12 & 1.2 \times 10^{-5} & 2.6 \times 10^{-3} \\ 13 & 1.2 \times 10^{-5} & 3.4 \times 10^{-3} \\ 14 & 1.1 \times 10^{-5} & 4.3 \times 10^{-3} \\ 15 & 1.6 \times 10^{-5} & 5.4 \times 10^{-3} \\ 16 & 2.2 \times 10^{-5} & 6.8 \times 10^{-3} \\ 17 & 2.2 \times 10^{-5} & 8.2 \times 10^{-3} \\ 18 & 7.9 \times 10^{-6} & 1.1 \times 10^{-2} \\ 19 & 3.7 \times 10^{-6} & 1.3 \times 10^{-2} \\ 20 & & \end{array}$




\begin{tabular}{|c|c|c|}
\hline \multirow[b]{2}{*}{ Series \# } & \multicolumn{2}{|c|}{${ }^{60}$ Co-Upper-Middle Section } \\
\hline & $\begin{array}{l}\text { Leach Rate } \\
\mathrm{g} / \mathrm{cm}^{2}-\text { day } \\
\end{array}$ & $\begin{array}{c}\text { Cumulative } \\
\text { Fraction Leached } \\
\end{array}$ \\
\hline 1 & $5.6 \times 10^{-5}$ & $1.2 \times 10^{-4}$ \\
\hline 2 & $1.6 \times 10^{-5}$ & $1.5 \times 10^{-4}$ \\
\hline 3 & $1.6 \times 10^{-5}$ & $1.8 \times 10^{-4}$ \\
\hline 4 & $1.8 \times 10^{-5}$ & $2.2 \times 10^{-4}$ \\
\hline 5 & $1.3 \times 10^{-5}$ & $3.3 \times 10^{-4}$ \\
\hline 6 & $9.8 \times 10^{-6}$ & $4.5 \times 10^{-4}$ \\
\hline 7 & $1.2 \times 10^{-5}$ & $6.2 \times 10^{-4}$ \\
\hline 8 & $1.5 \times 10^{-5}$ & $8.4 \times 10^{-4}$ \\
\hline 9 & $1.2 \times 10^{-5}$ & $1.0 \times 10^{-3}$ \\
\hline 10 & $1.3 \times 10^{-5}$ & $1.2 \times 10^{-3}$ \\
\hline 11 & $1.3 \times 10^{-5}$ & $1.4 \times 10^{-3}$ \\
\hline 12 & $1.4 \times 10^{-5}$ & $1.6 \times 10^{-3}$ \\
\hline 13 & $8.5 \times 10^{-6}$ & $2.1 \times 10^{-3}$ \\
\hline 14 & $1.2 \times 10^{-5}$ & $2.9 \times 10^{-3}$ \\
\hline 15 & $1.1 \times 10^{-5}$ & $3.6 \times 10^{-3}$ \\
\hline 16 & $1.4 \times 10^{-5}$ & $4.6 \times 10^{-3}$ \\
\hline 17 & $2.0 \times 10^{-5}$ & $5.8 \times 10^{-3}$ \\
\hline 18 & $1.8 \times 10^{-5}$ & $6.8 \times 10^{-3}$ \\
\hline 19 & $6.9 \times 10^{-6}$ & $9.4 \times 10^{-3}$ \\
\hline 20 & $3.1 \times 10^{-6}$ & $1.1 \times 10^{-2}$ \\
\hline
\end{tabular}




\section{${ }^{60}$ Co-Top Section}

$\underline{\text { Series \# }} \quad \begin{array}{r}\text { Leach Rate } \\ \mathrm{g} / \mathrm{cm}^{2} \text {-day }\end{array} \quad \begin{gathered}\text { Cumulative } \\ \text { Fraction Leached }\end{gathered}$

$\begin{array}{rll}1 & 1.5 \times 10^{-4} & 3.9 \times 10^{-4} \\ 2 & 2.6 \times 10^{-5} & 4.6 \times 10^{-4} \\ 3 & 2.4 \times 10^{-5} & 5.3 \times 10^{-4} \\ 4 & 2.7 \times 10^{-5} & 6.0 \times 10^{-4} \\ 5 & 1.8 \times 10^{-5} & 7.9 \times 10^{-4} \\ 6 & 1.8 \times 10^{-5} & 1.1 \times 10^{-3} \\ 7 & 2.0 \times 10^{-5} & 1.4 \times 10^{-3} \\ 8 & 2.3 \times 10^{-5} & 1.9 \times 10^{-3} \\ 9 & 2.0 \times 10^{-5} & 2.3 \times 10^{-3} \\ 10 & 1.9 \times 10^{-5} & 2.6 \times 10^{-3} \\ \text { * } & 2.1 \times 10^{-5} & 3.0 \times 10^{-3} \\ 11 & 2.5 \times 10^{-5} & 3.5 \times 10^{-3} \\ 12 & 1.9 \times 10^{-5} & 5.0 \times 10^{-3} \\ 13 & 2.3 \times 10^{-5} & 6.8 \times 10^{-3} \\ 14 & 2.5 \times 10^{-5} & 9.1 \times 10^{-3} \\ 15 & 2.8 \times 10^{-5} & 1.2 \times 10^{-2} \\ 16 & 3.4 \times 10^{-5} & 1.4 \times 10^{-2} \\ 17 & 3.2 \times 10^{-5} & 1.6 \times 10^{-2} \\ 18 & 1.2 \times 10^{-5} & 2.2 \times 10^{-2} \\ 19 & 8.3 \times 10^{-6} & 2.7 \times 10^{-2} \\ 20 & & \end{array}$


${ }^{90}$ Sr-Bottom Section

\begin{tabular}{|c|c|c|}
\hline Series \# & $\begin{array}{r}\text { Leach Rate } \\
\mathrm{g} / \mathrm{cm}^{2}-\text { day } \\
\end{array}$ & $\begin{array}{l}\text { Cumulative } \\
\text { Fraction Leached }\end{array}$ \\
\hline 1 & $7.8 \times 10^{-5}$ & $1.2 \times 10^{-4}$ \\
\hline 2 & $2.1 \times 10^{-5}$ & $1.6 \times 10^{-4}$ \\
\hline 3 & $2.4 \times 10^{-5}$ & $1.9 \times 10^{-4}$ \\
\hline 4 & $2.6 \times 10^{-5}$ & $2.3 \times 10^{-4}$ \\
\hline 5 & $6.5 \times 10^{-6}$ & $2.8 \times 10^{-4}$ \\
\hline 6 & $1.4 \times 10^{-5}$ & $4.0 \times 10^{-4}$ \\
\hline 7 & $1.4 \times 10^{-5}$ & $5.5 \times 10^{-4}$ \\
\hline 8 & $1.7 \times 10^{-5}$ & $7.4 \times 10^{-4}$ \\
\hline 9 & $7.3 \times 10^{-6}$ & $8.2 \times 10^{-4}$ \\
\hline 10 & $1.6 \times 10^{-5}$ & $1.0 \times 10^{-3}$ \\
\hline 11 & $1.8 \times 10^{-5}$ & $1.2 \times 10^{-3}$ \\
\hline 12 & $1.8 \times 10^{-5}$ & $1.4 \times 10^{-3}$ \\
\hline 13 & $1.5 \times 10^{-5}$ & $2.1 \times 10^{-3}$ \\
\hline 14 & $1.4 \times 10^{-5}$ & $2.8 \times 10^{-3}$ \\
\hline 15 & $1.6 \times 10^{-5}$ & $3.6 \times 10^{-3}$ \\
\hline 16 & $2.0 \times 10^{-5}$ & $4.6 \times 10^{-3}$ \\
\hline 17 & $2.4 \times 10^{-5}$ & $5.7 \times 10^{-3}$ \\
\hline 18 & $2.4 \times 10^{-5}$ & $6.8 \times 10^{-3}$ \\
\hline 19 & $2.6 \times 10^{-5}$ & $1.4 \times 10^{-2}$ \\
\hline 20 & $7.9 \times 10^{-6}$ & $1.7 \times 10^{-2}$ \\
\hline
\end{tabular}




\begin{tabular}{|c|c|c|}
\hline \multirow[b]{2}{*}{ Series \# } & \multicolumn{2}{|c|}{${ }^{90}$ Sr-Lower-Middle Section } \\
\hline & $\begin{array}{r}\text { Leach Rate } \\
\mathrm{g} / \mathrm{cm}^{2} \text {-day } \\
\end{array}$ & $\begin{array}{c}\text { Cumulative } \\
\text { Fraction Leached } \\
\end{array}$ \\
\hline 1 & $6.2 \times 10^{-5}$ & $1.4 \times 10^{-4}$ \\
\hline 2 & $1.4 \times 10^{-5}$ & $1.7 \times 10^{-4}$ \\
\hline 3 & $1.8 \times 10^{-5}$ & $2.1 \times 10^{-4}$ \\
\hline 4 & $1.9 \times 10^{-5}$ & $2.6 \times 10^{-4}$ \\
\hline 5 & $1.3 \times 10^{-5}$ & $3.8 \times 10^{-4}$ \\
\hline 6 & $1.3 \times 10^{-5}$ & $5.5 \times 10^{-4}$ \\
\hline 7 & $1.2 \times 10^{-5}$ & $7.4 \times 10^{-4}$ \\
\hline 8 & $1.5 \times 10^{-5}$ & $9.8 \times 10^{-4}$ \\
\hline 9 & $1.3 \times 10^{-5}$ & $1.2 \times 10^{-3}$ \\
\hline 10 & $1.6 \times 10^{-5}$ & $1.4 \times 10^{-3}$ \\
\hline * 11 & $1.4 \times 10^{-5}$ & $1.7 \times 10^{-3}$ \\
\hline 12 & $2.7 \times 10^{-5}$ & $2.1 \times 10^{-3}$ \\
\hline 13 & $1.2 \times 10^{-5}$ & $2.9 \times 10^{-3}$ \\
\hline 14 & $1.5 \times 10^{-5}$ & $3.9 \times 10^{-3}$ \\
\hline 15 & $1.5 \times 10^{-5}$ & $5.1 \times 10^{-3}$ \\
\hline 16 & $2.0 \times 10^{-5}$ & $6.5 \times 10^{-3}$ \\
\hline 17 & $2.4 \times 10^{-5}$ & $8.1 \times 10^{-3}$ \\
\hline 18 & $2.6 \times 10^{-5}$ & $9.8 \times 10^{-3}$ \\
\hline 19 & $1.9 \times 10^{-5}$ & $1.8 \times 10^{-2}$ \\
\hline 20 & $9.7 \times 10^{-7}$ & $1.8 \times 10^{-2}$ \\
\hline
\end{tabular}




\begin{tabular}{|c|c|c|}
\hline \multirow[b]{2}{*}{ Series \# } & \multicolumn{2}{|c|}{${ }^{90}$ Sr-Upper-Middle Section } \\
\hline & $\begin{array}{l}\text { Leach Rate } \\
\mathrm{g} / \mathrm{cm}^{2}-\text { day }\end{array}$ & $\begin{array}{l}\text { Cumulative } \\
\text { Fraction Leached } \\
\end{array}$ \\
\hline 1 & $6.7 \times 10^{-5}$ & $1.4 \times 10^{-4}$ \\
\hline 2 & $1.9 \times 10^{-5}$ & $1.8 \times 10^{-4}$ \\
\hline 3 & $1.4 \times 10^{-5}$ & $2.1 \times 10^{-4}$ \\
\hline 4 & $2.0 \times 10^{-5}$ & $2.5 \times 10^{-4}$ \\
\hline 5 & $1.3 \times 10^{-5}$ & $3.6 \times 10^{-4}$ \\
\hline 6 & $1.2 \times 10^{-5}$ & $5.1 \times 10^{-4}$ \\
\hline 7 & $1.3 \times 10^{-5}$ & $6.9 \times 10^{-4}$ \\
\hline 8 & $1.4 \times 10^{-5}$ & $9.0 \times 10^{-4}$ \\
\hline 9 & $1.2 \times 10^{-5}$ & $1.1 \times 10^{-3}$ \\
\hline 10 & $1.5 \times 10^{-5}$ & $1.3 \times 10^{-3}$ \\
\hline 11 & $1.4 \times 10^{-5}$ & $1.5 \times 10^{-3}$ \\
\hline 12 & $1.5 \times 10^{-5}$ & $1.7 \times 10^{-3}$ \\
\hline 13 & $1.1 \times 10^{-5}$ & $2.4 \times 10^{-3}$ \\
\hline 14 & $1.1 \times 10^{-5}$ & $3.1 \times 10^{-3}$ \\
\hline 15 & $1.2 \times 10^{-5}$ & $4.0 \times 10^{-3}$ \\
\hline 16 & $1.7 \times 10^{-5}$ & $5.1 \times 10^{-3}$ \\
\hline 17 & $2.1 \times 10^{-5}$ & $6.4 \times 10^{-3}$ \\
\hline 18 & $2.1 \times 10^{-5}$ & $7.6 \times 10^{-3}$ \\
\hline 19 & $1.6 \times 10^{-5}$ & $1.4 \times 10^{-2}$ \\
\hline 20 & $7.5 \times 10^{-7}$ & $1.4 \times 10^{-2}$ \\
\hline
\end{tabular}




\section{${ }^{90}$ Sr-Top Section}

\begin{tabular}{ccc} 
Series \# & $\begin{array}{r}\text { Leach Rate } \\
\mathrm{g} / \mathrm{cm}^{2} \text {-day }\end{array}$ & $\begin{array}{c}\text { Cumulative } \\
\text { Fraction Leached }\end{array}$ \\
\hline
\end{tabular}

\begin{tabular}{|c|c|c|}
\hline 1 & $2.5 \times 10^{-4}$ & $6.6 \times 10^{-4}$ \\
\hline 2 & $3.1 \times 10^{-5}$ & $7.5 \times 10^{-4}$ \\
\hline 3 & $2.5 \times 10^{-5}$ & $8.1 \times 10^{-4}$ \\
\hline 4 & $2.7 \times 10^{-5}$ & $8.8 \times 10^{-4}$ \\
\hline 5 & $1.7 \times 10^{-5}$ & $1.1 \times 10^{-3}$ \\
\hline 6 & $1.6 \times 10^{-5}$ & $1.3 \times 10^{-3}$ \\
\hline 7 & $1.7 \times 10^{-5}$ & $1.6 \times 10^{-3}$ \\
\hline 8 & $1.7 \times 10^{-5}$ & $2.0 \times 10^{-3}$ \\
\hline 9 & $1.9 \times 10^{-5}$ & $2.3 \times 10^{-3}$ \\
\hline 10 & $2.1 \times 10^{-5}$ & $2.7 \times 10^{-3}$ \\
\hline * 11 & $2.2 \times 10^{-5}$ & $3.1 \times 10^{-3}$ \\
\hline 12 & $1.9 \times 10^{-5}$ & $3.5 \times 10^{-3}$ \\
\hline 13 & $1.8 \times 10^{-5}$ & $4.9 \times 10^{-3}$ \\
\hline 14 & $2.0 \times 10^{-5}$ & $6.5 \times 10^{-3}$ \\
\hline 15 & $2.1 \times 10^{-5}$ & $8.4 \times 10^{-3}$ \\
\hline 16 & $2.6 \times 10^{-5}$ & $1.1 \times 10^{-2}$ \\
\hline 17 & $2.8 \times 10^{-5}$ & $1.3 \times 10^{-2}$ \\
\hline 18 & $3.0 \times 10^{-5}$ & $1.5 \times 10^{-2}$ \\
\hline 19 & $3.2 \times 10^{-5}$ & $3.0 \times 10^{-2}$ \\
\hline 20 & $1.5 \times 10^{-6}$ & $3.1 \times 10^{-2}$ \\
\hline
\end{tabular}




\section{$\underline{106}_{\text {Rh-Bottom Section }}$ (a)}

\begin{tabular}{ll} 
Series \# & $\begin{array}{l}\text { Leach Rate } \\
\mathrm{g} / \mathrm{cm}^{2} \text {-day }\end{array}$ \\
\hline
\end{tabular}

$\begin{array}{rl}1 & \\ 2 & 2.6 \times 10^{-7} \\ 3 & \\ 4 & 5.3 \times 10^{-7} \\ 5 & \\ 6 & 3.9 \times 10^{-8} \\ 7 & \\ 8 & 9.2 \times 10^{-8} \\ 9 & 7.8 \times 10^{-8} \\ 10 & 1.7 \times 10^{-7} \\ 11 & \\ 12 & \\ 13 & 1.2 \times 10^{-7} \\ 14 & \\ 15 & \\ 16 & 7.7 \times 10^{-8} \\ 17 & 2.6 \times 10^{-7} \\ 18 & 2.8 \times 10^{-7} \\ 19 & 2.7 \times 10^{-7} \\ 20 & 6.3 \times 10^{-8}\end{array}$

(a) ${ }^{106} \mathrm{Rh}$ is the daughter product of ${ }^{106} \mathrm{Ru}$.

(b) Blanks indicate that no data were obtained for that series. 


\section{${ }^{106}$ Rh-Lower-Middle Section $(a)$}

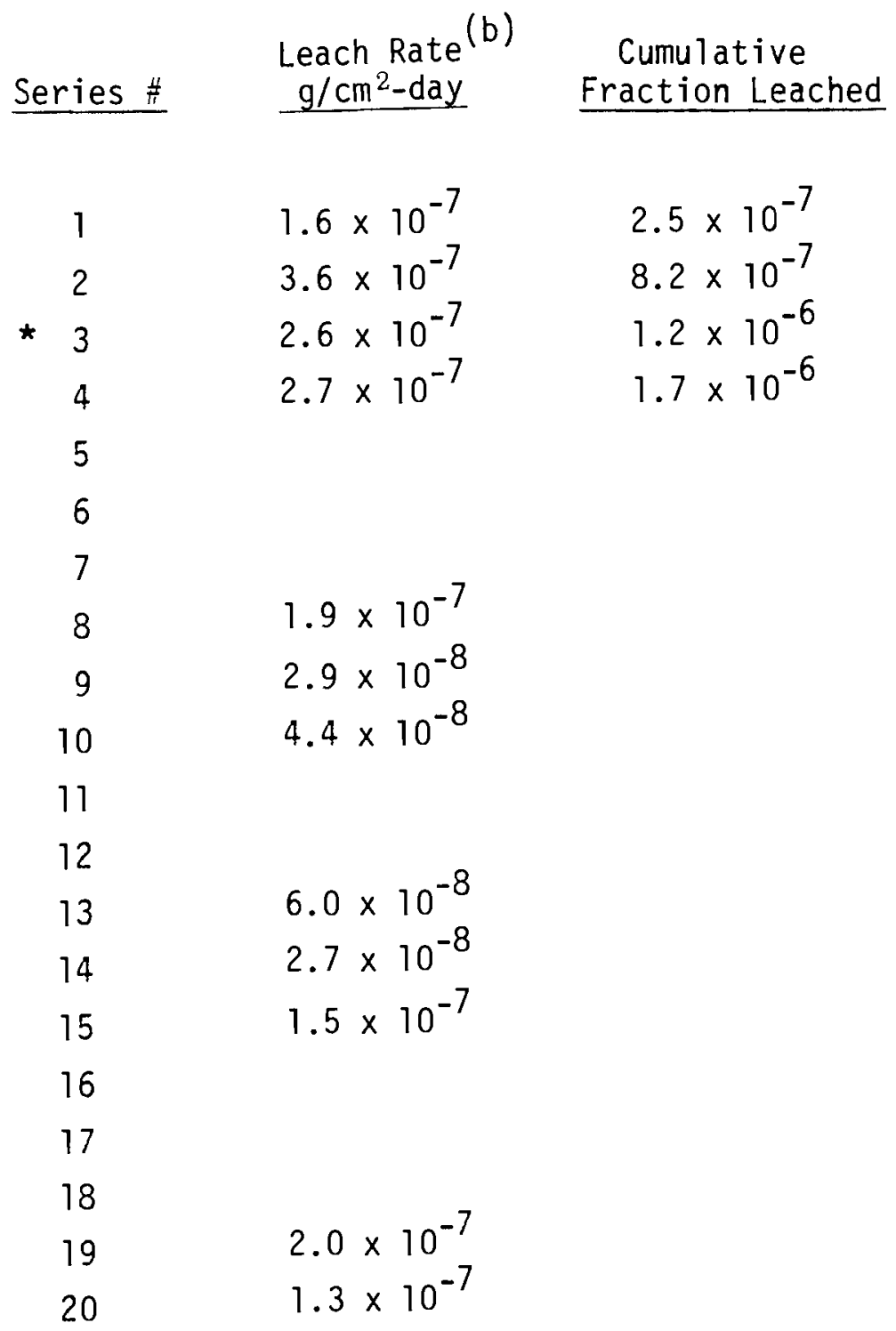

* Denotes that an analysis is not available for this series. The number is an average of the above series with the same leach time.

(a) $106 \mathrm{Rh}$ is the daughter product of $106 \mathrm{Ru}$.

(b) Blanks indicate that no data were obtained for that series. 


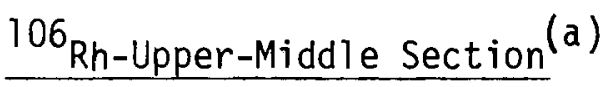

Series \# $\quad \begin{aligned} & \text { Leach Rate } \\ & \mathrm{g} / \mathrm{cm}^{2} \text {-day }\end{aligned}$

$\begin{array}{rl}1 & \\ 2 & 4.4 \times 10^{-7} \\ 3 & \\ 4 & 1.2 \times 10^{-7} \\ 5 & 1.8 \times 10^{-7} \\ 6 & 5.3 \times 10^{-7} \\ 7 & 1.3 \times 10^{-7} \\ 8 & \\ 9 & 9.4 \times 10^{-8} \\ 10 & 1.9 \times 10^{-7} \\ 11 & \\ 12 & \\ 13 & 5.5 \times 10^{-8} \\ 14 & 4.7 \times 10^{-8} \\ 15 & \\ 16 & 9.2 \times 10^{-8} \\ 17 & 2.2 \times 10^{-7} \\ 18 & \\ 19 & 1.4 \times 10^{-7} \\ 20 & 8.4 \times 10^{-8}\end{array}$

(a) ${ }^{106} \mathrm{Rh}$ is the daughter product of ${ }^{106} \mathrm{Ru}$.

(b) Blanks indicate that no data were obtained for that series. 


\section{${ }^{106}$ Rh-Top Section $(a)$}

\begin{tabular}{|c|c|c|}
\hline Series \# & $\begin{array}{l}\text { Leach Rate } \\
\mathrm{g} / \mathrm{cm}^{2} \text {-day }\end{array}$ & $\begin{array}{c}\text { Cumulative } \\
\text { Fraction Leached } \\
\end{array}$ \\
\hline 1 & $4.3 \times 10^{-6}$ & $1.1 \times 10^{-5}$ \\
\hline 2 & $8.8 \times 10^{-7}$ & $1.4 \times 10^{-5}$ \\
\hline 3 & $6.6 \times 10^{-7}$ & $1.6 \times 10^{-5}$ \\
\hline 4 & $9.8 \times 10^{-7}$ & $1.8 \times 10^{-5}$ \\
\hline \multicolumn{3}{|l|}{5} \\
\hline \multicolumn{3}{|l|}{6} \\
\hline 7 & $9.1 \times 10^{-8}$ & \\
\hline 8 & $3.3 \times 10^{-7}$ & \\
\hline 9 & $1.7 \times 10^{-7}$ & \\
\hline 10 & $8.6 \times 10^{-8}$ & \\
\hline \multicolumn{3}{|l|}{11} \\
\hline \multicolumn{3}{|l|}{12} \\
\hline 13 & $4.4 \times 10^{-8}$ & \\
\hline \multicolumn{3}{|l|}{14} \\
\hline 15 & $3.6 \times 10^{-7}$ & \\
\hline \multicolumn{3}{|l|}{16} \\
\hline \multicolumn{3}{|l|}{17} \\
\hline 18 & $2.5 \times 10^{-7}$ & \\
\hline 19 & $4.8 \times 10^{-7}$ & \\
\hline 20 & $2.7 \times 10^{-7}$ & \\
\hline
\end{tabular}




\section{${ }^{125}$ Sb-Bottom Section}

\begin{tabular}{|c|c|c|}
\hline Series \# & $\begin{array}{r}\text { Leach Rate } \\
\mathrm{g} / \mathrm{cm}^{2}-\text { day }\end{array}$ & $\begin{array}{c}\text { Cumulative } \\
\text { Fraction Leached } \\
\end{array}$ \\
\hline 1 & $4.7 \times 10^{-5}$ & $7.4 \times 10^{-5}$ \\
\hline 2 & $2.8 \times 10^{-5}$ & $1.2 \times 10^{-4}$ \\
\hline 3 & $2.9 \times 10^{-5}$ & $1.6 \times 10^{-4}$ \\
\hline 4 & $2.9 \times 10^{-5}$ & $2.1 \times 10^{-4}$ \\
\hline 5 & $1.5 \times 10^{-5}$ & $3.0 \times 10^{-4}$ \\
\hline 6 & $1.2 \times 10^{-5}$ & $4.2 \times 10^{-4}$ \\
\hline 7 & $9.9 \times 10^{-6}$ & $5.2 \times 10^{-4}$ \\
\hline 8 & $1.4 \times 10^{-5}$ & $6.7 \times 10^{-4}$ \\
\hline 9 & $1.2 \times 10^{-5}$ & $8.0 \times 10^{-4}$ \\
\hline 10 & $1.5 \times 10^{-5}$ & $9.7 \times 10^{-4}$ \\
\hline 11 & $1.4 \times 10^{-5}$ & $1.1 \times 10^{-3}$ \\
\hline 12 & $1.4 \times 10^{-5}$ & $1.3 \times 10^{-3}$ \\
\hline 13 & $9.6 \times 10^{-6}$ & $1.7 \times 10^{-3}$ \\
\hline 14 & $1.0 \times 10^{-5}$ & $2.2 \times 10^{-3}$ \\
\hline 15 & $9.7 \times 10^{-6}$ & $2.7 \times 10^{-3}$ \\
\hline 16 & $1.1 \times 10^{-5}$ & $3.3 \times 10^{-3}$ \\
\hline 17 & $1.6 \times 10^{-5}$ & $4.0 \times 10^{-3}$ \\
\hline 18 & $1.5 \times 10^{-5}$ & $4.7 \times 10^{-3}$ \\
\hline 19 & $2.6 \times 10^{-6}$ & $5.5 \times 10^{-3}$ \\
\hline 20 & $1.7 \times 10^{-6}$ & $6.0 \times 10^{-3}$ \\
\hline
\end{tabular}


${ }^{125}$ Sb-Lower-Middle Section

\begin{tabular}{lll} 
Series \# & $\begin{array}{c}\text { Leach Rate } \\
\mathrm{g} / \mathrm{cm}^{2} \text {-day }\end{array}$ & $\begin{array}{c}\text { Cumulative } \\
\text { Fraction Leached }\end{array}$ \\
\cline { 3 - 3 } 1 & $4.1 \times 10^{-5}$ & $9.3 \times 10^{-5}$ \\
2 & $2.1 \times 10^{-5}$ & $1.4 \times 10^{-4}$ \\
3 & $2.4 \times 10^{-5}$ & $2.0 \times 10^{-4}$ \\
4 & $2.3 \times 10^{-5}$ & $2.5 \times 10^{-4}$ \\
5 & $1.4 \times 10^{-5}$ & $3.7 \times 10^{-4}$ \\
6 & $1.1 \times 10^{-5}$ & $5.3 \times 10^{-4}$ \\
7 & $1.1 \times 10^{-5}$ & $6.9 \times 10^{-4}$ \\
8 & $1.2 \times 10^{-5}$ & $8.8 \times 10^{-4}$ \\
9 & $1.2 \times 10^{-5}$ & $1.1 \times 10^{-3}$ \\
10 & $1.2 \times 10^{-5}$ & $1.3 \times 10^{-3}$ \\
* & $1.2 \times 10^{-5}$ & $1.4 \times 10^{-3}$ \\
11 & $2.1 \times 10^{-5}$ & $1.8 \times 10^{-3}$ \\
12 & $1.0 \times 10^{-5}$ & $2.5 \times 10^{-3}$ \\
13 & $1.2 \times 10^{-5}$ & $3.2 \times 10^{-3}$ \\
14 & $1.0 \times 10^{-5}$ & $4.0 \times 10^{-3}$ \\
15 & $1.2 \times 10^{-5}$ & $4.9 \times 10^{-3}$ \\
16 & $1.8 \times 10^{-5}$ & $6.1 \times 10^{-3}$ \\
17 & $1.9 \times 10^{-5}$ & $7.3 \times 10^{-3}$ \\
18 & $3.4 \times 10^{-6}$ & $8.7 \times 10^{-3}$ \\
19 & $2.0 \times 10^{-6}$ & $9.7 \times 10^{-3}$ \\
20 & &
\end{tabular}




\section{Sb-Upper-Middle Section}

\begin{tabular}{|c|c|c|}
\hline Series \# & $\begin{array}{l}\text { Leach Rate } \\
\mathrm{g} / \mathrm{cm}^{2}-\text { day }\end{array}$ & $\begin{array}{c}\text { Cumulative } \\
\text { Fraction Leached }\end{array}$ \\
\hline 1 & $4.0 \times 10^{-3}$ & $8.4 \times 10^{-5}$ \\
\hline 2 & $2.2 \times 10^{-3}$ & $1.3 \times 10^{-4}$ \\
\hline 3 & $2.3 \times 10^{-3}$ & $1.8 \times 10^{-4}$ \\
\hline 4 & $2.4 \times 10^{-3}$ & $2.3 \times 10^{-4}$ \\
\hline 5 & $1.5 \times 10^{-3}$ & $3.5 \times 10^{-4}$ \\
\hline 6 & $9.8 \times 10^{-6}$ & $4.7 \times 10^{-4}$ \\
\hline 7 & $1.1 \times 10^{-5}$ & $6.3 \times 10^{-4}$ \\
\hline 8 & $1.4 \times 10^{-5}$ & $8.3 \times 10^{-4}$ \\
\hline 9 & $8.2 \times 10^{-6}$ & $9.5 \times 10^{-4}$ \\
\hline 10 & $9.2 \times 10^{-6}$ & $1.1 \times 10^{-3}$ \\
\hline 11 & $1.3 \times 10^{-5}$ & $1.3 \times 10^{-3}$ \\
\hline 12 & $1.4 \times 10^{-5}$ & $1.6 \times 10^{-3}$ \\
\hline 13 & $7.5 \times 10^{-6}$ & $2.0 \times 10^{-3}$ \\
\hline 14 & $1.0 \times 10^{-5}$ & $2.7 \times 10^{-3}$ \\
\hline 15 & $9.5 \times 10^{-6}$ & $3.3 \times 10^{-3}$ \\
\hline 16 & $1.2 \times 10^{-5}$ & $4.2 \times 10^{-3}$ \\
\hline 17 & $1.5 \times 10^{-5}$ & $5.1 \times 10^{-3}$ \\
\hline 18 & $1.5 \times 10^{-5}$ & $5.9 \times 10^{-3}$ \\
\hline 19 & $3.5 \times 10^{-6}$ & $7.3 \times 10^{-3}$ \\
\hline 20 & $1.7 \times 10^{-6}$ & $8.0 \times 10^{-3}$ \\
\hline
\end{tabular}




\section{${ }^{125}$ Sb-Top Section}

\begin{tabular}{ccc} 
Series \# & $\begin{array}{rll}\text { Leach Rate } \\
\mathrm{g} / \mathrm{cm}^{2}-\text { day }\end{array}$ & $\begin{array}{c}\text { Cumulative } \\
\text { Fraction Leached }\end{array}$ \\
\cline { 1 - 1 } 1 & $2.2 \times 10^{-4}$ & $5.7 \times 10^{-4}$ \\
2 & $9.1 \times 10^{-5}$ & $8.1 \times 10^{-4}$ \\
3 & $6.7 \times 10^{-5}$ & $9.9 \times 10^{-4}$ \\
4 & $5.2 \times 10^{-5}$ & $1.1 \times 10^{-3}$ \\
5 & $3.7 \times 10^{-5}$ & $1.5 \times 10^{-3}$ \\
6 & $2.2 \times 10^{-5}$ & $1.9 \times 10^{-3}$ \\
7 & $2.0 \times 10^{-5}$ & $2.2 \times 10^{-3}$ \\
8 & $1.9 \times 10^{-5}$ & $2.6 \times 10^{-3}$ \\
9 & $1.9 \times 10^{-5}$ & $3.0 \times 10^{-3}$ \\
10 & $2.0 \times 10^{-5}$ & $3.3 \times 10^{-3}$ \\
$* 11$ & $2.0 \times 10^{-5}$ & $3.7 \times 10^{-3}$ \\
12 & $2.4 \times 10^{-5}$ & $4.1 \times 10^{-3}$ \\
13 & $1.8 \times 10^{-5}$ & $5.6 \times 10^{-3}$ \\
14 & $2.1 \times 10^{-5}$ & $7.3 \times 10^{-3}$ \\
15 & $1.9 \times 10^{-5}$ & $9.0 \times 10^{-3}$ \\
16 & $2.0 \times 10^{-5}$ & $1.1 \times 10^{-2}$ \\
17 & $2.1 \times 10^{-5}$ & $1.2 \times 10^{-2}$ \\
18 & $2.1 \times 10^{-5}$ & $1.4 \times 10^{-2}$ \\
19 & $7.9 \times 10^{-6}$ & $1.8 \times 10^{-2}$ \\
20 & $5.1 \times 10^{-6}$ & $2.1 \times 10^{-2}$ \\
& &
\end{tabular}

* Denotes that an analysis is not available for this series. The number is an average of the above series with the same leach time. 


\section{${ }^{134}$ Cs-Bottom Section}

\begin{tabular}{|c|c|c|}
\hline Series \# & $\begin{array}{l}\text { Leach Rate } \\
\mathrm{g} / \mathrm{cm}^{2} \text {-day }\end{array}$ & $\begin{array}{c}\text { Cumulative } \\
\text { Fraction Leached }\end{array}$ \\
\hline 1 & $1.0 \times 10^{-4}$ & $1.6 \times 10^{-4}$ \\
\hline 2 & $2.8 \times 10^{-5}$ & $2.1 \times 10^{-4}$ \\
\hline 3 & $3.3 \times 10^{-5}$ & $2.6 \times 10^{-4}$ \\
\hline 4 & $3.4 \times 10^{-5}$ & $3.1 \times 10^{-4}$ \\
\hline 5 & $1.8 \times 10^{-5}$ & $4.3 \times 10^{-4}$ \\
\hline 6 & $2.0 \times 10^{-5}$ & $6.2 \times 10^{-4}$ \\
\hline 7 & $1.9 \times 10^{-5}$ & $8.3 \times 10^{-4}$ \\
\hline 8 & $2.3 \times 10^{-5}$ & $1.1 \times 10^{-3}$ \\
\hline 9 & $2.2 \times 10^{-5}$ & $1.3 \times 10^{-3}$ \\
\hline 10 & $2.3 \times 10^{-5}$ & $1.6 \times 10^{-3}$ \\
\hline 11 & $2.6 \times 10^{-5}$ & $1.9 \times 10^{-3}$ \\
\hline *12 & $2.3 \times 10^{-5}$ & $2.1 \times 10^{-3}$ \\
\hline 13 & $2.2 \times 10^{-5}$ & $3.1 \times 10^{-3}$ \\
\hline 14 & $2.1 \times 10^{-5}$ & $4.1 \times 10^{-3}$ \\
\hline 15 & $2.2 \times 10^{-5}$ & $5.3 \times 10^{-3}$ \\
\hline 16 & $3.0 \times 10^{-5}$ & $6.8 \times 10^{-3}$ \\
\hline 17 & $5.6 \times 10^{-5}$ & $9.3 \times 10^{-3}$ \\
\hline 18 & $5.3 \times 10^{-5}$ & $1.2 \times 10^{-2}$ \\
\hline 19 & $2.7 \times 10^{-5}$ & $1.9 \times 10^{-2}$ \\
\hline 20 & $8.3 \times 10^{-6}$ & $2.2 \times 10^{-2}$ \\
\hline
\end{tabular}




\section{${ }^{134}$ Cs-Lower-Middle Section}

\begin{tabular}{|c|c|c|}
\hline Series \# & $\begin{array}{l}\text { Leach Rate } \\
\mathrm{g} / \mathrm{cm}^{2}-\text { day }\end{array}$ & $\begin{array}{c}\text { Cumulative } \\
\text { Fraction Leached }\end{array}$ \\
\hline 1 & $8.6 \times 10^{-5}$ & $2.0 \times 10^{-4}$ \\
\hline 2 & $2.1 \times 10^{-5}$ & $2.4 \times 10^{-4}$ \\
\hline 3 & $2.6 \times 10^{-5}$ & $3.0 \times 10^{-4}$ \\
\hline 4 & $2.8 \times 10^{-5}$ & $3.7 \times 10^{-4}$ \\
\hline 5 & $1.9 \times 10^{-5}$ & $5.4 \times 10^{-4}$ \\
\hline 6 & $1.9 \times 10^{-5}$ & $7.9 \times 10^{-4}$ \\
\hline 7 & $1.8 \times 10^{-5}$ & $1.1 \times 10^{-3}$ \\
\hline 8 & $2.2 \times 10^{-5}$ & $1.4 \times 10^{-3}$ \\
\hline 9 & $2.1 \times 10^{-5}$ & $1.8 \times 10^{-3}$ \\
\hline 10 & $2.2 \times 10^{-5}$ & $2.1 \times 10^{-3}$ \\
\hline *11 & $2.1 \times 10^{-5}$ & $2.4 \times 10^{-3}$ \\
\hline$\star 12$ & $2.1 \times 10^{-5}$ & $2.8 \times 10^{-3}$ \\
\hline 13 & $1.9 \times 10^{-5}$ & $4.1 \times 10^{-3}$ \\
\hline 14 & $2.4 \times 10^{-5}$ & $5.7 \times 10^{-3}$ \\
\hline 15 & $2.4 \times 10^{-5}$ & $7.5 \times 10^{-3}$ \\
\hline 16 & $3.3 \times 10^{-5}$ & $9.9 \times 10^{-3}$ \\
\hline 17 & $5.8 \times 10^{-5}$ & $1.4 \times 10^{-2}$ \\
\hline 18 & $5.8 \times 10^{-5}$ & $1.7 \times 10^{-2}$ \\
\hline 19 & $2.2 \times 10^{-5}$ & $2.6 \times 10^{-2}$ \\
\hline 20 & $1.0 \times 10^{-5}$ & $3.1 \times 10^{-2}$ \\
\hline
\end{tabular}

* Denotes that an analysis is not available for this series. The number is an average of the above series with the same leach time. 


\section{${ }^{134} \mathrm{Cs}$-Upper-Middle Section}

\begin{tabular}{|c|c|c|}
\hline Series \# & $\begin{array}{l}\text { Leach Rate } \\
\mathrm{g} / \mathrm{cm}^{2} \text {-day }\end{array}$ & $\begin{array}{c}\text { Cumulative } \\
\text { Fraction Leached }\end{array}$ \\
\hline 1 & $9.3 \times 10^{-5}$ & $1.9 \times 10^{-4}$ \\
\hline 2 & $2.9 \times 10^{-5}$ & $2.5 \times 10^{-4}$ \\
\hline 3 & $2.8 \times 10^{-5}$ & $3.1 \times 10^{-4}$ \\
\hline 4 & $2.9 \times 10^{-5}$ & $3.7 \times 10^{-4}$ \\
\hline 5 & $1.8 \times 10^{-5}$ & $5.3 \times 10^{-4}$ \\
\hline 6 & $1.8 \times 10^{-5}$ & $7.5 \times 10^{-4}$ \\
\hline 7 & $1.9 \times 10^{-5}$ & $1.0 \times 10^{-3}$ \\
\hline 8 & $2.1 \times 10^{-5}$ & $1.3 \times 10^{-3}$ \\
\hline 9 & $1.5 \times 10^{-5}$ & $1.6 \times 10^{-3}$ \\
\hline 10 & $2.1 \times 10^{-5}$ & $1.9 \times 10^{-3}$ \\
\hline 11 & $2.2 \times 10^{-5}$ & $2.2 \times 10^{-3}$ \\
\hline 12 & $2.3 \times 10^{-5}$ & $2.5 \times 10^{-3}$ \\
\hline 13 & $1.5 \times 10^{-5}$ & $3.5 \times 10^{-3}$ \\
\hline 14 & $1.8 \times 10^{-5}$ & $4.6 \times 10^{-3}$ \\
\hline 15 & $2.0 \times 10^{-5}$ & $6.0 \times 10^{-3}$ \\
\hline 16 & $2.8 \times 10^{-5}$ & $7.9 \times 10^{-3}$ \\
\hline 17 & $5.0 \times 10^{-5}$ & $1.1 \times 10^{-2}$ \\
\hline 18 & $4.4 \times 10^{-5}$ & $1.3 \times 10^{-2}$ \\
\hline 19 & $2.0 \times 10^{-5}$ & $2.1 \times 10^{-2}$ \\
\hline 0 & $1.4 \times 10^{-5}$ & $2.7 \times 10^{-2}$ \\
\hline
\end{tabular}




\section{${ }^{134}$ Cs-Top Section}

\begin{tabular}{ccc} 
Series \# & $\begin{array}{c}\text { Leach Rate } \\
\mathrm{g} / \mathrm{cm}^{2}-\text { day }\end{array}$ & $\begin{array}{c}\text { Cumulative } \\
\text { Fraction Leached }\end{array}$ \\
\cline { 1 - 2 } 1 & $1.1 \times 10^{-4}$ & $2.0 \times 10^{-4}$ \\
2 & $2.2 \times 10^{-5}$ & $2.4 \times 10^{-4}$ \\
3 & $2.1 \times 10^{-5}$ & $4.0 \times 10^{-4}$ \\
4 & $2.3 \times 10^{-5}$ & $4.6 \times 10^{-4}$ \\
5 & $1.5 \times 10^{-5}$ & $6.2 \times 10^{-4}$ \\
6 & $1.5 \times 10^{-5}$ & $8.6 \times 10^{-4}$ \\
7 & $1.8 \times 10^{-5}$ & $1.2 \times 10^{-3}$ \\
8 & $2.0 \times 10^{-5}$ & $1.6 \times 10^{-3}$ \\
9 & $2.3 \times 10^{-5}$ & $2.0 \times 10^{-3}$ \\
10 & $2.4 \times 10^{-5}$ & $2.4 \times 10^{-3}$ \\
11 & $2.8 \times 10^{-5}$ & $3.0 \times 10^{-3}$ \\
$* 12$ & $2.3 \times 10^{-5}$ & $3.4 \times 10^{-3}$ \\
13 & $2.5 \times 10^{-5}$ & $5.4 \times 10^{-3}$ \\
14 & $3.1 \times 10^{-5}$ & $7.8 \times 10^{-3}$ \\
15 & $3.1 \times 10^{-5}$ & $1.1 \times 10^{-2}$ \\
16 & $4.0 \times 10^{-5}$ & $1.4 \times 10^{-2}$ \\
17 & $6.0 \times 10^{-5}$ & $1.9 \times 10^{-2}$ \\
18 & $6.4 \times 10^{-5}$ & $2.3 \times 10^{-2}$ \\
19 & $3.0 \times 10^{-5}$ & $3.8 \times 10^{-2}$ \\
20 & $2.0 \times 10^{-5}$ & $4.9 \times 10^{-2}$
\end{tabular}

* Denotes that an analysis is not available for this series. The number is an average of the above series with the same leach time. 
${ }^{137}$ Cs-Bottom Section

\begin{tabular}{|c|c|c|}
\hline Series \# & $\begin{array}{l}\text { Leach Rate } \\
\mathrm{g} / \mathrm{cm}^{2} \text {-day }\end{array}$ & $\begin{array}{c}\text { Cumulative } \\
\text { Fraction Leached } \\
\end{array}$ \\
\hline 1 & $1.1 \times 10^{-4}$ & $1.7 \times 10^{-4}$ \\
\hline 2 & $2.8 \times 10^{-5}$ & $2.1 \times 10^{-4}$ \\
\hline 3 & $3.4 \times 10^{-5}$ & $2.7 \times 10^{-4}$ \\
\hline 4 & $3.5 \times 10^{-5}$ & $3.2 \times 10^{-4}$ \\
\hline 5 & $1.8 \times 10^{-5}$ & $4.3 \times 10^{-4}$ \\
\hline 6 & $2.0 \times 10^{-5}$ & $6.5 \times 10^{-4}$ \\
\hline 7 & $2.0 \times 10^{-5}$ & $8.5 \times 10^{-4}$ \\
\hline 8 & $2.2 \times 10^{-5}$ & $1.1 \times 10^{-3}$ \\
\hline 9 & $2.2 \times 10^{-5}$ & $1.4 \times 10^{-3}$ \\
\hline 10 & $2.4 \times 10^{-5}$ & $1.6 \times 10^{-3}$ \\
\hline 11 & $2.6 \times 10^{-5}$ & $1.9 \times 10^{-3}$ \\
\hline$\star 12$ & $2.3 \times 10^{-5}$ & $2.1 \times 10^{-3}$ \\
\hline 13 & $2.1 \times 10^{-5}$ & $3.0 \times 10^{-3}$ \\
\hline 14 & $2.1 \times 10^{-5}$ & $4.0 \times 10^{-3}$ \\
\hline 15 & $2.2 \times 10^{-5}$ & $5.0 \times 10^{-3}$ \\
\hline 16 & $2.9 \times 10^{-5}$ & $6.5 \times 10^{-3}$ \\
\hline 17 & $3.5 \times 10^{-5}$ & $8.0 \times 10^{-3}$ \\
\hline 18 & $3.3 \times 10^{-5}$ & $9.5 \times 10^{-3}$ \\
\hline 19 & $1.8 \times 10^{-5}$ & $1.5 \times 10^{-2}$ \\
\hline 20 & $5.7 \times 10^{-6}$ & $1.7 \times 10^{-2}$ \\
\hline
\end{tabular}

* Denotes that an analysis is not available for this series. The number is an average of the above series with the same leach time. 


\begin{tabular}{|c|c|c|}
\hline \multirow[b]{2}{*}{ Series \# } & \multicolumn{2}{|c|}{${ }^{7}$ Cs-Lower-Middle Section } \\
\hline & $\begin{array}{l}\text { Leach Rate } \\
\mathrm{g} / \mathrm{cm}^{2} \text {-day }\end{array}$ & $\begin{array}{c}\text { Cumulative } \\
\text { Fraction Leached }\end{array}$ \\
\hline 1 & $8.6 \times 10^{-5}$ & $2.0 \times 10^{-4}$ \\
\hline 2 & $2.2 \times 10^{-5}$ & $2.5 \times 10^{-4}$ \\
\hline 3 & $2.7 \times 10^{-5}$ & $3.1 \times 10^{-4}$ \\
\hline 4 & $2.9 \times 10^{-5}$ & $3.7 \times 10^{-4}$ \\
\hline 5 & $1.9 \times 10^{-5}$ & $5.5 \times 10^{-4}$ \\
\hline 6 & $1.9 \times 10^{-5}$ & $8.0 \times 10^{-4}$ \\
\hline 7 & $1.9 \times 10^{-5}$ & $1.1 \times 10^{-3}$ \\
\hline 8 & $2.2 \times 10^{-5}$ & $1.5 \times 10^{-3}$ \\
\hline 9 & $2.1 \times 10^{-5}$ & $1.8 \times 10^{-3}$ \\
\hline 10 & $2.2 \times 10^{-5}$ & $2.2 \times 10^{-3}$ \\
\hline$\star 11$ & $2.1 \times 10^{-5}$ & $2.5 \times 10^{-3}$ \\
\hline$\star 12$ & $2.1 \times 10^{-5}$ & $2.8 \times 10^{-3}$ \\
\hline 13 & $1.9 \times 10^{-5}$ & $4.1 \times 10^{-3}$ \\
\hline 14 & $2.3 \times 10^{-5}$ & $5.5 \times 10^{-3}$ \\
\hline 15 & $2.3 \times 10^{-5}$ & $7.5 \times 10^{-3}$ \\
\hline 16 & $3.3 \times 10^{-5}$ & $1.0 \times 10^{-2}$ \\
\hline 17 & $3.6 \times 10^{-5}$ & $1.2 \times 10^{-2}$ \\
\hline 18 & $3.6 \times 10^{-5}$ & $1.5 \times 10^{-2}$ \\
\hline 19 & $1.5 \times 10^{-5}$ & $2.1 \times 10^{-2}$ \\
\hline 20 & $7.2 \times 10^{-6}$ & $2.4 \times 10^{-2}$ \\
\hline
\end{tabular}

* Denotes that an analysis is not available for this series. The number is an average of the above series with the same leach time. 
${ }^{137}$ Cs-Upper-Middle Section

\begin{tabular}{ccc} 
Series \# & $\begin{array}{c}\text { Leach Rate } \\
\mathrm{g} / \mathrm{cm}^{2} \text {-day }\end{array}$ & $\begin{array}{c}\text { Cumulative } \\
\text { Fraction Leached }\end{array}$ \\
\cline { 1 - 2 } 1 & $9.1 \times 10^{-5}$ & $1.9 \times 10^{-4}$ \\
2 & $2.0 \times 10^{-5}$ & $2.6 \times 10^{-4}$ \\
3 & $2.9 \times 10^{-5}$ & $3.2 \times 10^{-4}$ \\
4 & $3.1 \times 10^{-5}$ & $3.8 \times 10^{-4}$ \\
5 & $1.9 \times 10^{-5}$ & $5.5 \times 10^{-4}$ \\
6 & $1.9 \times 10^{-5}$ & $7.5 \times 10^{-4}$ \\
7 & $2.0 \times 10^{-5}$ & $1.1 \times 10^{-3}$ \\
8 & $2.1 \times 10^{-5}$ & $1.4 \times 10^{-3}$ \\
9 & $1.6 \times 10^{-5}$ & $1.6 \times 10^{-3}$ \\
10 & $2.1 \times 10^{-5}$ & $1.9 \times 10^{-3}$ \\
11 & $2.2 \times 10^{-5}$ & $2.2 \times 10^{-3}$ \\
12 & $2.3 \times 10^{-5}$ & $2.6 \times 10^{-3}$ \\
13 & $1.5 \times 10^{-5}$ & $3.5 \times 10^{-3}$ \\
14 & $1.8 \times 10^{-5}$ & $4.6 \times 10^{-3}$ \\
15 & $1.9 \times 10^{-5}$ & $6.0 \times 10^{-3}$ \\
16 & $2.7 \times 10^{-5}$ & $8.0 \times 10^{-3}$ \\
17 & $3.0 \times 10^{-5}$ & $9.5 \times 10^{-3}$ \\
18 & $2.6 \times 10^{-5}$ & $1.1 \times 10^{-2}$ \\
19 & $1.2 \times 10^{-5}$ & $1.6 \times 10^{-2}$ \\
20 & $5.7 \times 10^{-6}$ & $1.8 \times 10^{-2}$
\end{tabular}




\section{${ }^{137}$ Cs-Top Section}

\begin{tabular}{ccc} 
Series \# & $\begin{array}{r}\text { Leach Rate } \\
\mathrm{g} / \mathrm{cm}^{2} \text {-day }\end{array}$ & $\begin{array}{c}\text { Cumulative } \\
\text { Fraction Leached }\end{array}$ \\
\hline 1 & $1.1 \times 10^{-4}$ & $2.9 \times 10^{-4}$ \\
2 & $2.3 \times 10^{-5}$ & $3.5 \times 10^{-4}$ \\
3 & $2.2 \times 10^{-5}$ & $4.1 \times 10^{-4}$ \\
4 & $2.3 \times 10^{-5}$ & $4.7 \times 10^{-4}$ \\
5 & $1.5 \times 10^{-5}$ & $6.5 \times 10^{-4}$ \\
6 & $1.6 \times 10^{-5}$ & $9.0 \times 10^{-4}$ \\
7 & $1.8 \times 10^{-5}$ & $1.2 \times 10^{-3}$ \\
8 & $2.0 \times 10^{-5}$ & $1.6 \times 10^{-3}$ \\
9 & $2.4 \times 10^{-5}$ & $2.1 \times 10^{-3}$ \\
10 & $2.5 \times 10^{-5}$ & $2.5 \times 10^{-3}$ \\
11 & $2.9 \times 10^{-5}$ & $3.0 \times 10^{-3}$ \\
$* 12$ & $2.3 \times 10^{-5}$ & $3.5 \times 10^{-3}$ \\
13 & $2.4 \times 10^{-5}$ & $5.5 \times 10^{-3}$ \\
14 & $3.1 \times 10^{-5}$ & $8.0 \times 10^{-3}$ \\
15 & $3.0 \times 10^{-5}$ & $1.1 \times 10^{-2}$ \\
16 & $4.0 \times 10^{-5}$ & $1.4 \times 10^{-2}$ \\
17 & $3.8 \times 10^{-5}$ & $1.7 \times 10^{-2}$ \\
18 & $3.9 \times 10^{-5}$ & $2.0 \times 10^{-2}$ \\
19 & $1.9 \times 10^{-5}$ & $2.9 \times 10^{-2}$ \\
20 & $8.5 \times 10^{-6}$ & $3.4 \times 10^{-2}$ \\
& &
\end{tabular}

* Denotes that an analysis is not available for this series. The number is an average of the above series with the same leach time. 


\section{${ }^{144}$ Ce-Bottom Section}

\begin{tabular}{ccc} 
Series \# & $\begin{array}{c}\text { Leach Rate } \\
\mathrm{g} / \mathrm{cm}^{2} \text {-day }\end{array}$ & $\begin{array}{c}\text { Cumulative } \\
\text { Fraction Leached }\end{array}$ \\
\cline { 2 - 3 } 1 & $2.0 \times 10^{-6}$ & $3.2 \times 10^{-6}$ \\
2 & $3.0 \times 10^{-7}$ & $3.6 \times 10^{-6}$ \\
3 & $2.1 \times 10^{-7}$ & $4.0 \times 10^{-6}$ \\
4 & $1.7 \times 10^{-7}$ & $4.3 \times 10^{-6}$ \\
5 & $3.3 \times 10^{-8}$ & $4.5 \times 10^{-6}$ \\
6 & $1.3 \times 10^{-8}$ & $4.6 \times 10^{-6}$ \\
7 & $3.7 \times 10^{-9}$ & $4.6 \times 10^{-6}$ \\
8 & $2.2 \times 10^{-8}$ & $4.9 \times 10^{-6}$ \\
9 & $1.5 \times 10^{-8}$ & $5.0 \times 10^{-6}$ \\
10 & $1.4 \times 10^{-8}$ & $5.2 \times 10^{-6}$ \\
11 & $4.6 \times 10^{-9}$ & $5.2 \times 10^{-6}$ \\
12 & $2.8 \times 10^{-8}$ & $5.5 \times 10^{-6}$ \\
13 & $1.6 \times 10^{-8}$ & $6.3 \times 10^{-6}$ \\
14 & $2.7 \times 10^{-8}$ & $7.6 \times 10^{-6}$ \\
15 & $3.0 \times 10^{-8}$ & $9.2 \times 10^{-6}$ \\
16 & $2.5 \times 10^{-8}$ & $1.0 \times 10^{-5}$ \\
17 & $5.3 \times 10^{-8}$ & $1.3 \times 10^{-5}$ \\
18 & $8.7 \times 10^{-8}$ & $1.7 \times 10^{-5}$ \\
19 & $5.4 \times 10^{-8}$ & $3.2 \times 10^{-5}$ \\
20 & $3.6 \times 10^{-9}$ & $3.4 \times 10^{-5}$
\end{tabular}




\section{${ }^{144}$ Ce-Lower-Middle Section}

\begin{tabular}{ll} 
Series \# & $\begin{array}{r}\text { Leach Rate } \\
\mathrm{g} / \mathrm{cm}^{2} \text {-day }\end{array} \quad \begin{array}{c}\text { Cumulative } \\
\text { Fraction Leached }\end{array}$ \\
\hline
\end{tabular}

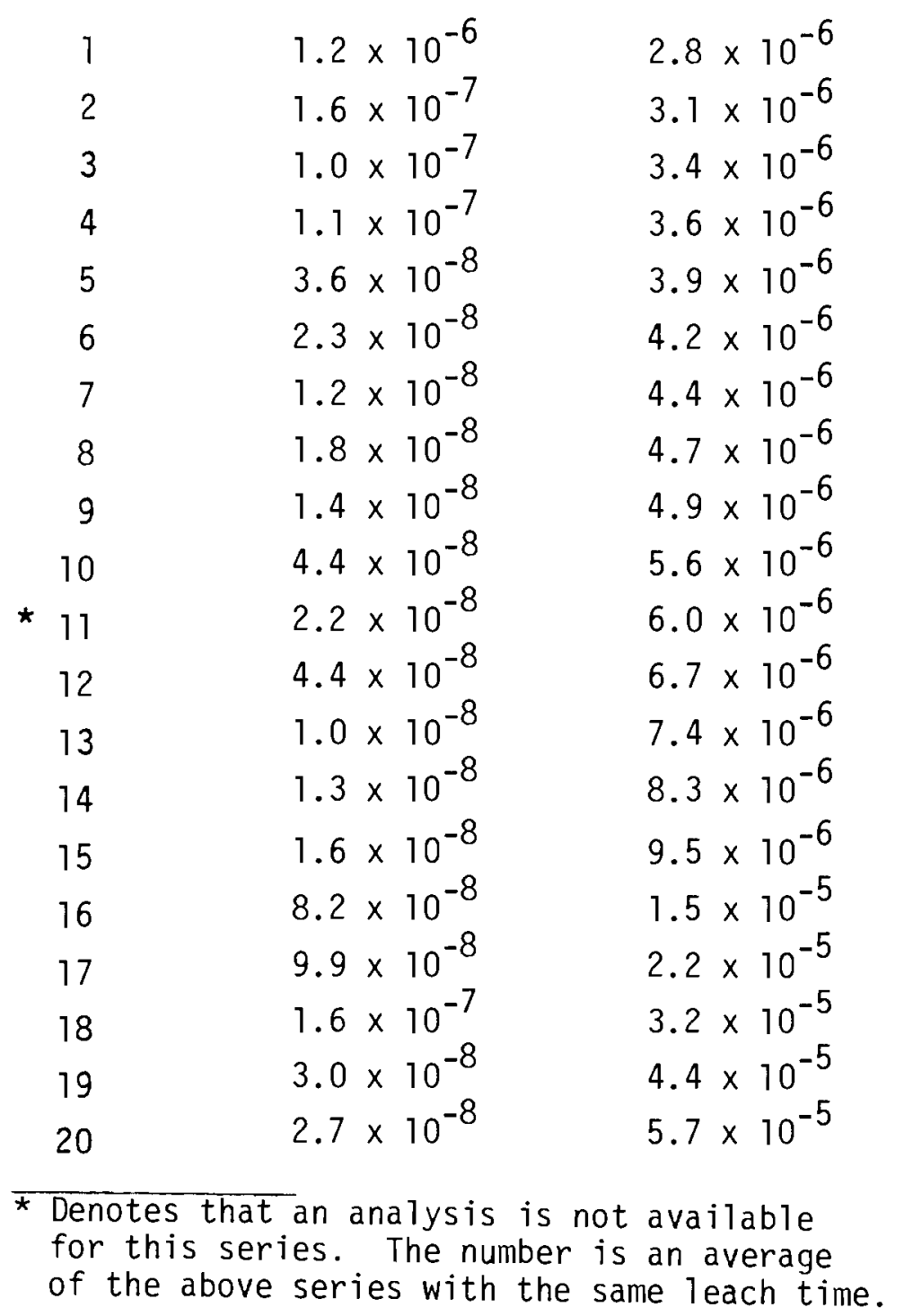




\begin{tabular}{ccc} 
& \multicolumn{2}{l}{${ }^{144}$ Ce-Upper-Middle Section } \\
\cline { 2 - 3 } Series \# & $\begin{array}{c}\text { Leach Rate } \\
\mathrm{g} / \mathrm{cm}^{2} \text {-day }\end{array}$ & $\begin{array}{c}\text { Cumulative } \\
\text { Fraction Leached }\end{array}$ \\
\cline { 3 - 3 } 1 & $1.5 \times 10^{-6}$ & $3.0 \times 10^{-6}$ \\
2 & $3.7 \times 10^{-7}$ & $3.8 \times 10^{-6}$ \\
3 & $3.5 \times 10^{-7}$ & $4.5 \times 10^{-6}$ \\
4 & $2.4 \times 10^{-7}$ & $5.0 \times 10^{-6}$ \\
5 & $4.2 \times 10^{-8}$ & $5.4 \times 10^{-6}$ \\
6 & $2.7 \times 10^{-8}$ & $5.7 \times 10^{-6}$ \\
7 & $6.2 \times 10^{-9}$ & $5.8 \times 10^{-6}$ \\
8 & $1.2 \times 10^{-8}$ & $6.0 \times 10^{-6}$ \\
9 & $1.4 \times 10^{-8}$ & $6.2 \times 10^{-6}$ \\
10 & $3.3 \times 10^{-8}$ & $6.7 \times 10^{-6}$ \\
11 & $1.4 \times 10^{-8}$ & $6.9 \times 10^{-6}$ \\
12 & $8.5 \times 10^{-9}$ & $7.0 \times 10^{-6}$ \\
13 & $2.2 \times 10^{-8}$ & $8.4 \times 10^{-6}$ \\
14 & $1.0 \times 10^{-8}$ & $9.0 \times 10^{-6}$ \\
15 & $1.1 \times 10^{-8}$ & $9.8 \times 10^{-6}$ \\
16 & $7.3 \times 10^{-8}$ & $1.5 \times 10^{-5}$ \\
17 & $7.5 \times 10^{-8}$ & $1.9 \times 10^{-5}$ \\
18 & $7.9 \times 10^{-8}$ & $2.4 \times 10^{-5}$ \\
19 & $3.1 \times 10^{-8}$ & $3.5 \times 10^{-5}$ \\
20 & $4.3 \times 10^{-9}$ & $3.7 \times 10^{-5}$
\end{tabular}




\section{${ }^{144}$ Ce-Top Section}

Series \# $\quad \begin{gathered}\text { Leach Rate } \\ \mathrm{g} / \mathrm{cm}^{2} \text {-day }\end{gathered} \quad \begin{gathered}\text { Cumulative } \\ \text { Fraction Leached }\end{gathered}$

$\begin{array}{lll}1 & 3.8 \times 10^{-6} & 1.0 \times 10^{-5} \\ 2 & 2.9 \times 10^{-7} & 1.1 \times 10^{-5} \\ 3 & 2.0 \times 10^{-7} & 1.1 \times 10^{-5} \\ 4 & 2.6 \times 10^{-7} & 1.2 \times 10^{-5} \\ 5 & 5.1 \times 10^{-8} & 1.3 \times 10^{-5} \\ 6 & 2.2 \times 10^{-8} & 1.3 \times 10^{-5} \\ 7 & 2.1 \times 10^{-8} & 1.3 \times 10^{-5} \\ 8 & 3.4 \times 10^{-8} & 1.4 \times 10^{-5} \\ 9 & 1.6 \times 10^{-8} & 1.4 \times 10^{-5} \\ 10 & 4.5 \times 10^{-8} & 1.5 \times 10^{-5} \\ \text { * } 11 & 2.9 \times 10^{-8} & 1.6 \times 10^{-5} \\ \text { * } 12 & 2.9 \times 10^{-8} & 1.6 \times 10^{-5} \\ \text { * } 13 & 2.9 \times 10^{-8} & 1.7 \times 10^{-5} \\ 14 & 2.1 \times 10^{-8} & 1.8 \times 10^{-5} \\ \text { * } 15 & 2.1 \times 10^{-8} & 2.0 \times 10^{-5} \\ 16 & 2.7 \times 10^{-8} & 2.2 \times 10^{-5} \\ 17 & 3.8 \times 10^{-8} & 2.5 \times 10^{-5} \\ 18 & 5.7 \times 10^{-8} & 2.9 \times 10^{-5} \\ 18 & 2.1 \times 10^{-8} & 4.0 \times 10^{-5} \\ 19 & 4.3 \times 10^{-8} & 6.4 \times 10^{-5} \\ 20 & & \end{array}$




\section{${ }^{154}$ Eu-Bottom Section}

\section{Leach Rate Cumulative}

Series \# $\mathrm{g} / \mathrm{cm}^{2}$-day $\quad$ Fraction Leached

$\begin{array}{lll}1 & 1.9 \times 10^{-6} & 3.1 \times 10^{-6} \\ 2 & 3.1 \times 10^{-7} & 3.6 \times 10^{-6} \\ 3 & 3.3 \times 10^{-7} & 4.1 \times 10^{-6} \\ 4 & 2.2 \times 10^{-7} & 4.4 \times 10^{-6} \\ 5 & 1.4 \times 10^{-7} & 5.3 \times 10^{-6} \\ 6 & 1.4 \times 10^{-7} & 6.6 \times 10^{-6} \\ 7 & 1.3 \times 10^{-7} & 8.1 \times 10^{-6} \\ 8 & 1.6 \times 10^{-7} & 9.9 \times 10^{-6} \\ 9 & 1.1 \times 10^{-7} & 1.1 \times 10^{-5} \\ 10 & 9.2 \times 10^{-8} & 1.2 \times 10^{-5} \\ 11 & 1.9 \times 10^{-7} & 1.4 \times 10^{-5} \\ 12 & 2.1 \times 10^{-7} & 1.6 \times 10^{-5} \\ 13 & 1.5 \times 10^{-7} & 2.3 \times 10^{-5} \\ 14 & 1.8 \times 10^{-7} & 3.2 \times 10^{-5} \\ 15 & 2.9 \times 10^{-7} & 4.8 \times 10^{-5} \\ 16 & 3.4 \times 10^{-7} & 6.5 \times 10^{-5} \\ 17 & 4.5 \times 10^{-7} & 8.5 \times 10^{-5} \\ 18 & 6.2 \times 10^{-7} & 1.1 \times 10^{-4} \\ 19 & 2.5 \times 10^{-7} & 1.9 \times 10^{-4} \\ 20 & 9.4 \times 10^{-8} & 2.2 \times 10^{-4}\end{array}$




\section{$154_{\text {Eu-Lower-Middle Section }}$}

Series \# $\quad \begin{array}{rc}\text { Leach Rate } \\ \mathrm{g} / \mathrm{cm}^{2} \text {-day }\end{array} \quad \begin{gathered}\text { Cumulative } \\ \text { Fraction Leached }\end{gathered}$

$\begin{array}{lll}1 & 1.2 \times 10^{-6} & 2.8 \times 10^{-6} \\ 2 & 2.7 \times 10^{-7} & 3.4 \times 10^{-6} \\ 3 & 3.1 \times 10^{-7} & 4.1 \times 10^{-6} \\ 4 & 1.9 \times 10^{-7} & 4.6 \times 10^{-6} \\ 5 & 1.5 \times 10^{-7} & 5.9 \times 10^{-6} \\ 6 & 1.3 \times 10^{-7} & 8.2 \times 10^{-6} \\ 7 & 1.6 \times 10^{-7} & 1.1 \times 10^{-5} \\ 8 & 9.2 \times 10^{-8} & 1.2 \times 10^{-5} \\ 9 & 1.1 \times 10^{-7} & 1.4 \times 10^{-5} \\ 10 & 9.0 \times 10^{-8} & 1.5 \times 10^{-5} \\ 11 & 1.1 \times 10^{-7} & 1.7 \times 10^{-5} \\ 12 & 4.3 \times 10^{-7} & 2.4 \times 10^{-5} \\ 13 & 1.9 \times 10^{-7} & 3.7 \times 10^{-5} \\ 14 & 3.9 \times 10^{-7} & 6.3 \times 10^{-5} \\ 15 & 3.8 \times 10^{-7} & 9.3 \times 10^{-5} \\ 16 & 6.5 \times 10^{-7} & 1.4 \times 10^{-4} \\ 17 & 9.8 \times 10^{-7} & 2.0 \times 10^{-4} \\ 18 & 1.3 \times 10^{-6} & 2.9 \times 10^{-4} \\ 19 & 2.2 \times 10^{-7} & 3.8 \times 10^{-4} \\ 20 & 1.4 \times 10^{-7} & 4.5 \times 10^{-4}\end{array}$




\begin{tabular}{|c|c|c|}
\hline \multirow[b]{2}{*}{ Series \# } & \multicolumn{2}{|c|}{ Eu-Upper-Middle Section } \\
\hline & $\begin{array}{l}\text { Leach Rate } \\
\mathrm{g} / \mathrm{cm}^{2}-\text { day } \\
\end{array}$ & $\begin{array}{c}\text { Cumulative } \\
\text { Fraction Leached } \\
\end{array}$ \\
\hline 1 & $1.5 \times 10^{-6}$ & $3.2 \times 10^{-6}$ \\
\hline 2 & $3.7 \times 10^{-7}$ & $4.0 \times 10^{-6}$ \\
\hline 3 & $4.5 \times 10^{-7}$ & $4.9 \times 10^{-6}$ \\
\hline 4 & $2.8 \times 10^{-7}$ & $5.5 \times 10^{-6}$ \\
\hline 5 & $1.3 \times 10^{-7}$ & $6.6 \times 10^{-6}$ \\
\hline 6 & $1.5 \times 10^{-7}$ & $8.4 \times 10^{-6}$ \\
\hline 7 & $2.5 \times 10^{-7}$ & $1.2 \times 10^{-5}$ \\
\hline 8 & $2.6 \times 10^{-7}$ & $1.6 \times 10^{-5}$ \\
\hline 9 & $2.5 \times 10^{-7}$ & $1.9 \times 10^{-5}$ \\
\hline 10 & $1.7 \times 10^{-7}$ & $2.2 \times 10^{-5}$ \\
\hline 11 & $1.3 \times 10^{-7}$ & $2.4 \times 10^{-5}$ \\
\hline 12 & $9.0 \times 10^{-8}$ & $2.5 \times 10^{-5}$ \\
\hline 13 & $1.5 \times 10^{-7}$ & $3.4 \times 10^{-5}$ \\
\hline 14 & $2.2 \times 10^{-7}$ & $4.8 \times 10^{-5}$ \\
\hline 15 & $3.3 \times 10^{-7}$ & $7.1 \times 10^{-5}$ \\
\hline 16 & $6.7 \times 10^{-7}$ & $1.2 \times 10^{-4}$ \\
\hline 17 & $7.4 \times 10^{-7}$ & $1.6 \times 10^{-4}$ \\
\hline 18 & $8.9 \times 10^{-7}$ & $2.1 \times 10^{-4}$ \\
\hline 19 & $1.9 \times 10^{-7}$ & $2.9 \times 10^{-4}$ \\
\hline 20 & $9.8 \times 10^{-8}$ & $3.3 \times 10^{-4}$ \\
\hline
\end{tabular}




\section{$154_{\text {Eu-Top Section }}^{10}$}

Series \# $\quad \begin{array}{r}\text { Leach Rate } \\ \mathrm{g} / \mathrm{cm}^{2} \text {-day }\end{array} \quad \begin{gathered}\text { Cumulative } \\ \text { Fraction Leached }\end{gathered}$

$\begin{array}{rll}1 & 4.7 \times 10^{-6} & 1.2 \times 10^{-5} \\ 2 & 7.6 \times 10^{-7} & 1.4 \times 10^{-5} \\ 3 & 6.6 \times 10^{-7} & 1.6 \times 10^{-5} \\ 4 & 7.3 \times 10^{-7} & 1.8 \times 10^{-5} \\ 5 & 3.8 \times 10^{-7} & 2.2 \times 10^{-5} \\ 6 & 1.7 \times 10^{-7} & 2.5 \times 10^{-5} \\ 7 & 3.1 \times 10^{-7} & 3.1 \times 10^{-5} \\ 8 & 3.3 \times 10^{-7} & 3.6 \times 10^{-5} \\ 9 & 2.2 \times 10^{-7} & 4.0 \times 10^{-5} \\ 10 & 1.4 \times 10^{-7} & 4.3 \times 10^{-5} \\ 11 & 2.5 \times 10^{-7} & 4.8 \times 10^{-5} \\ 12 & 1.2 \times 10^{-7} & 5.0 \times 10^{-5} \\ 13 & 1.2 \times 10^{-7} & 5.9 \times 10^{-5} \\ 14 & 1.2 \times 10^{-7} & 6.9 \times 10^{-5} \\ 15 & 2.0 \times 10^{-7} & 8.7 \times 10^{-5} \\ 16 & 1.6 \times 10^{-7} & 1.0 \times 10^{-4} \\ 17 & 3.5 \times 10^{-7} & 1.3 \times 10^{-4} \\ 18 & 5.7 \times 10^{-7} & 1.7 \times 10^{-4} \\ 19 & 1.4 \times 10^{-7} & 2.4 \times 10^{-4} \\ 20 & 9.0 \times 10^{-8} & 2.9 \times 10^{-4}\end{array}$




\begin{tabular}{|c|c|c|}
\hline \multirow[b]{2}{*}{ Series \# } & \multicolumn{2}{|c|}{${ }^{155}$ Eu-Bottom Section } \\
\hline & $\begin{array}{l}\text { Leach Rate } \\
\mathrm{g} / \mathrm{cm}^{2}-\text { day }\end{array}$ & $\begin{array}{c}\text { Cumulative } \\
\text { Fraction Leached } \\
\end{array}$ \\
\hline 1 & $1.8 \times 10^{-6}$ & $2.9 \times 10^{-6}$ \\
\hline 2 & $2.5 \times 10^{-7}$ & $3.3 \times 10^{-6}$ \\
\hline 3 & $4.2 \times 10^{-7}$ & $3.9 \times 10^{-6}$ \\
\hline 4 & $1.7 \times 10^{-7}$ & $4.2 \times 10^{-6}$ \\
\hline 5 & $1.6 \times 10^{-7}$ & $5.2 \times 10^{-6}$ \\
\hline 6 & $2.2 \times 10^{-7}$ & $7.3 \times 10^{-6}$ \\
\hline 7 & $1.1 \times 10^{-7}$ & $8.5 \times 10^{-6}$ \\
\hline 8 & $1.6 \times 10^{-7}$ & $1.0 \times 10^{-5}$ \\
\hline 9 & $8.2 \times 10^{-8}$ & $1.1 \times 10^{-5}$ \\
\hline 10 & $2.4 \times 10^{-7}$ & $1.4 \times 10^{-5}$ \\
\hline 11 & $1.5 \times 10^{-7}$ & $1.5 \times 10^{-5}$ \\
\hline 12 & $2.5 \times 10^{-7}$ & $1.8 \times 10^{-5}$ \\
\hline 13 & $1.3 \times 10^{-7}$ & $2.4 \times 10^{-5}$ \\
\hline 14 & $2.6 \times 10^{-7}$ & $3.7 \times 10^{-5}$ \\
\hline 15 & $3.0 \times 10^{-7}$ & $5.3 \times 10^{-5}$ \\
\hline 16 & $3.2 \times 10^{-7}$ & $6.9 \times 10^{-5}$ \\
\hline 17 & $4.7 \times 10^{-7}$ & $9.0 \times 10^{-5}$ \\
\hline 18 & $7.0 \times 10^{-7}$ & $1.2 \times 10^{-4}$ \\
\hline 19 & & \\
\hline 20 & & \\
\hline
\end{tabular}

(a) BTanks indicate that no data were obtained for that series. 


\begin{tabular}{|c|c|c|}
\hline \multirow[b]{2}{*}{ Series \# } & \multicolumn{2}{|c|}{ Eu-Lower-Middle Section } \\
\hline & $\begin{array}{l}\text { Leach Rate }{ }^{(a)} \\
\mathrm{g} / \mathrm{cm}^{2}-\text { day }\end{array}$ & $\begin{array}{l}\text { Cumulative } \\
\text { Fraction Leached }\end{array}$ \\
\hline 1 & $9.2 \times 10^{-7}$ & $2.2 \times 10^{-6}$ \\
\hline 2 & $2.5 \times 10^{-7}$ & $2.8 \times 10^{-6}$ \\
\hline 3 & $1.7 \times 10^{-7}$ & $3.2 \times 10^{-6}$ \\
\hline 4 & $1.7 \times 10^{-7}$ & $3.6 \times 10^{-6}$ \\
\hline 5 & $1.5 \times 10^{-7}$ & $5.0 \times 10^{-6}$ \\
\hline 6 & $2.0 \times 10^{-7}$ & $7.9 \times 10^{-6}$ \\
\hline 7 & $1.2 \times 10^{-7}$ & $9.8 \times 10^{-6}$ \\
\hline 8 & $6.2 \times 10^{-8}$ & $1.1 \times 10^{-5}$ \\
\hline 9 & $9.4 \times 10^{-8}$ & $1.2 \times 10^{-5}$ \\
\hline 10 & $8.1 \times 10^{-8}$ & $1.4 \times 10^{-5}$ \\
\hline * 11 & $8.9 \times 10^{-8}$ & $1.5 \times 10^{-5}$ \\
\hline 12 & $3.8 \times 10^{-7}$ & $2.1 \times 10^{-5}$ \\
\hline 13 & $2.2 \times 10^{-7}$ & $3.7 \times 10^{-5}$ \\
\hline 14 & $2.7 \times 10^{-7}$ & $5.6 \times 10^{-5}$ \\
\hline 15 & $3.1 \times 10^{-7}$ & $8.1 \times 10^{-5}$ \\
\hline 16 & $5.7 \times 10^{-7}$ & $1.2 \times 10^{-4}$ \\
\hline 17 & $9.9 \times 10^{-7}$ & $1.9 \times 10^{-4}$ \\
\hline 18 & $1.2 \times 10^{-6}$ & $2.7 \times 10^{-4}$ \\
\hline \multicolumn{3}{|l|}{19} \\
\hline \multicolumn{3}{|l|}{20} \\
\hline \multicolumn{3}{|c|}{$\begin{array}{l}\text { Denotes that an analysis is not available } \\
\text { for this series. The number is an average } \\
\text { of the above series with the same leach time } \\
\text { Blanks indicate that no data were obtained } \\
\text { for that series. }\end{array}$} \\
\hline
\end{tabular}




\begin{tabular}{|c|c|c|}
\hline \multirow[b]{2}{*}{ Series \# } & \multicolumn{2}{|c|}{ Eu-Upper-Middle Section } \\
\hline & $\begin{array}{l}\text { Leach Rate } \\
\mathrm{g} / \mathrm{cm}^{2}-\text { day }\end{array}$ & $\begin{array}{c}\text { Cumulative } \\
\text { Fraction Leached } \\
\end{array}$ \\
\hline 1 & $6.7 \times 10^{-7}$ & $1.5 \times 10^{-6}$ \\
\hline 2 & $3.5 \times 10^{-7}$ & $2.2 \times 10^{-6}$ \\
\hline 3 & $4.0 \times 10^{-7}$ & $3.1 \times 10^{-6}$ \\
\hline 4 & $4.8 \times 10^{-7}$ & $4.1 \times 10^{-6}$ \\
\hline 5 & $9.3 \times 10^{-8}$ & $4.9 \times 10^{-6}$ \\
\hline 6 & $1.1 \times 10^{-7}$ & $6.3 \times 10^{-6}$ \\
\hline 7 & $1.6 \times 10^{-7}$ & $8.8 \times 10^{-6}$ \\
\hline 8 & $1.6 \times 10^{-7}$ & $1.1 \times 10^{-5}$ \\
\hline 9 & $2.3 \times 10^{-7}$ & $1.5 \times 10^{-5}$ \\
\hline 10 & $1.8 \times 10^{-7}$ & $1.7 \times 10^{-5}$ \\
\hline 11 & $1.9 \times 10^{-8}$ & $2.0 \times 10^{-5}$ \\
\hline 12 & $1.1 \times 10^{-7}$ & $2.2 \times 10^{-5}$ \\
\hline 13 & $1.6 \times 10^{-7}$ & $3.3 \times 10^{-5}$ \\
\hline 14 & $2.5 \times 10^{-7}$ & $4.9 \times 10^{-5}$ \\
\hline 15 & $3.3 \times 10^{-7}$ & $7.3 \times 10^{-5}$ \\
\hline 16 & $6.0 \times 10^{-7}$ & $1.1 \times 10^{-4}$ \\
\hline 17 & $7.9 \times 10^{-7}$ & $1.6 \times 10^{-4}$ \\
\hline 18 & $9.0 \times 10^{-7}$ & $2.2 \times 10^{-4}$ \\
\hline 19 & & \\
\hline 20 & & \\
\hline
\end{tabular}

(a) Blanks indicate that no data were obtained for that series. 


\section{${ }^{155}$ Eu-Top Section}

Series \#

Leach Rate ${ }^{(a)}$

Cumulative $\mathrm{g} / \mathrm{cm}^{2}$-day

Fraction Leached

$\begin{array}{rrl}1 & 6.1 \times 10^{-6} & 1.7 \times 10^{-5} \\ 2 & 5.2 \times 10^{-7} & 1.8 \times 10^{-5} \\ 3 & 6.2 \times 10^{-7} & 2.0 \times 10^{-5} \\ 4 & 6.6 \times 10^{-7} & 2.2 \times 10^{-5} \\ 5 & 3.6 \times 10^{-7} & 2.6 \times 10^{-5} \\ 6 & 2.2 \times 10^{-7} & 2.9 \times 10^{-5} \\ 7 & 3.3 \times 10^{-7} & 3.6 \times 10^{-5} \\ 8 & 2.6 \times 10^{-7} & 4.1 \times 10^{-5} \\ 9 & 3.3 \times 10^{-7} & 4.7 \times 10^{-5} \\ 10 & 7.9 \times 10^{-8} & 4.9 \times 10^{-5} \\ * 11 & 2.5 \times 10^{-7} & 5.3 \times 10^{-5} \\ 12 & 2.7 \times 10^{-7} & 5.9 \times 10^{-5} \\ 13 & 5.6 \times 10^{-8} & 6.3 \times 10^{-5} \\ 14 & 5.1 \times 10^{-8} & 6.7 \times 10^{-5} \\ 15 & 4.4 \times 10^{-8} & 7.1 \times 10^{-5} \\ 16 & 2.9 \times 10^{-7} & 9.7 \times 10^{-5} \\ 17 & 3.2 \times 10^{-7} & 1.2 \times 10^{-4} \\ 18 & 6.4 \times 10^{-7} & 1.7 \times 10^{-4} \\ 19 & & \end{array}$




\begin{tabular}{|c|c|c|}
\hline \multirow[b]{2}{*}{ Series \# } & \multicolumn{2}{|c|}{$39+240$ Pu-Bottom Section } \\
\hline & $\begin{array}{l}\text { Leach Rate } \\
\mathrm{g} / \mathrm{cm}^{2} \text {-day }\end{array}$ & $\begin{array}{c}\text { Cumulative } \\
\text { Fraction Leached }\end{array}$ \\
\hline 1 & $6.8 \times 10^{-6}$ & $1.1 \times 10^{-5}$ \\
\hline 2 & $3.7 \times 10^{-6}$ & $1.7 \times 10^{-5}$ \\
\hline 3 & $4.8 \times 10^{-6}$ & $2.4 \times 10^{-5}$ \\
\hline 4 & $5.6 \times 10^{-6}$ & $3.3 \times 10^{-5}$ \\
\hline 5 & $1.8 \times 10^{-6}$ & $4.4 \times 10^{-5}$ \\
\hline 6 & $2.1 \times 10^{-6}$ & $6.4 \times 10^{-5}$ \\
\hline 7 & $2.4 \times 10^{-6}$ & $9.0 \times 10^{-5}$ \\
\hline 8 & $2.7 \times 10^{-6}$ & $1.2 \times 10^{-4}$ \\
\hline 9 & $3.1 \times 10^{-6}$ & $1.6 \times 10^{-4}$ \\
\hline 10 & $2.8 \times 10^{-6}$ & $1.9 \times 10^{-4}$ \\
\hline 11 & $3.7 \times 10^{-6}$ & $2.3 \times 10^{-4}$ \\
\hline 12 & $2.8 \times 10^{-6}$ & $2.6 \times 10^{-4}$ \\
\hline 13 & $1.2 \times 10^{-6}$ & $3.2 \times 10^{-4}$ \\
\hline 14 & $2.2 \times 10^{-6}$ & $4.2 \times 10^{-4}$ \\
\hline 15 & $1.2 \times 10^{-6}$ & $4.9 \times 10^{-4}$ \\
\hline 16 & $2.4 \times 10^{-6}$ & $6.1 \times 10^{-4}$ \\
\hline 17 & $1.5 \times 10^{-6}$ & $6.8 \times 10^{-4}$ \\
\hline 18 & $2.7 \times 10^{-6}$ & $8.0 \times 10^{-4}$ \\
\hline 19 & $5.2 \times 10^{-7}$ & $9.5 \times 10^{-4}$ \\
\hline 20 & $8.8 \times 10^{-7}$ & $1.3 \times 10^{-3}$ \\
\hline
\end{tabular}




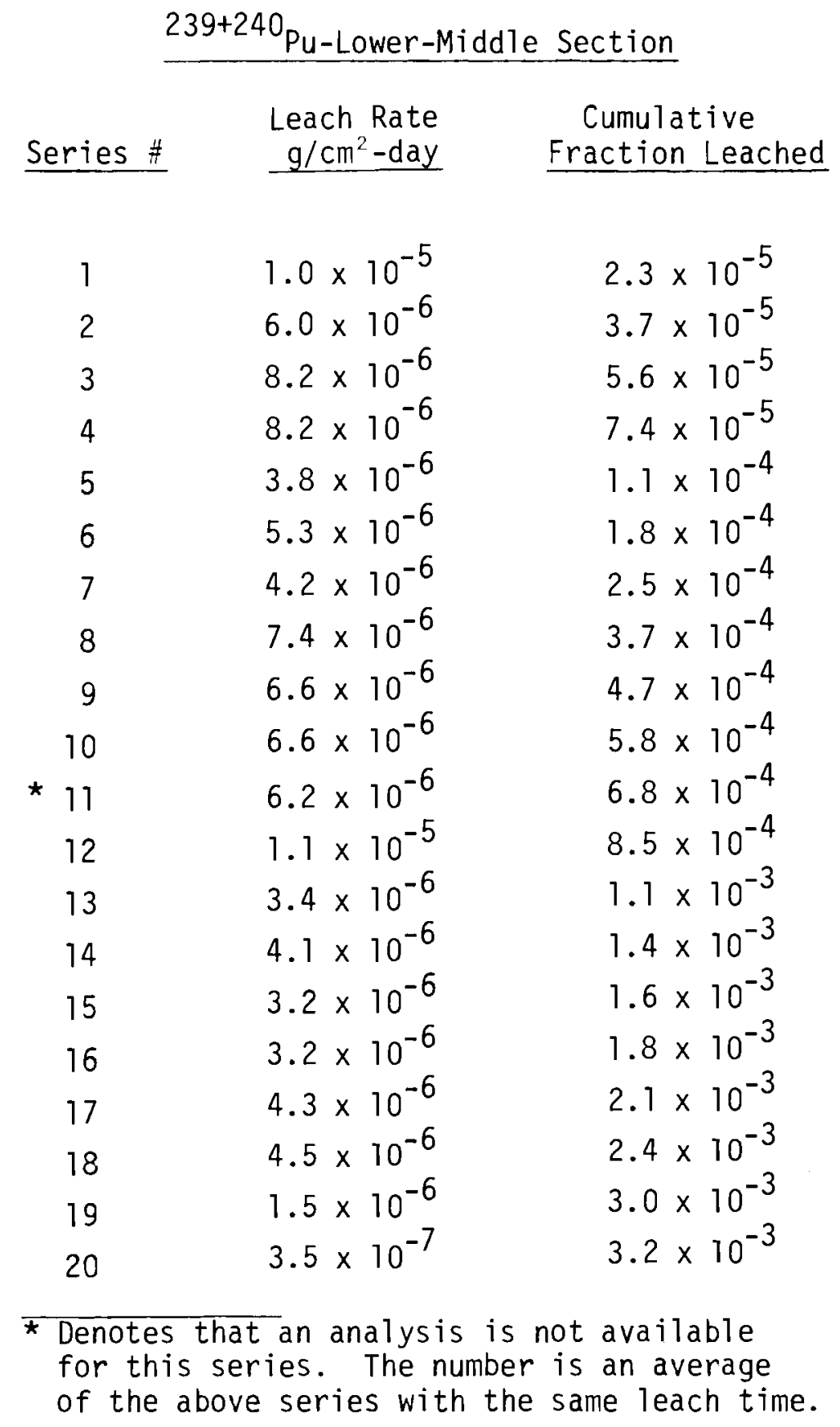




\section{${ }^{239+240}$ Pu-Upper-Middle Section}

Series \# $\quad \begin{array}{r}\text { Leach Rate } \\ \mathrm{g} / \mathrm{cm}^{2} \text {-day }\end{array} \quad \begin{gathered}\text { Cumulative } \\ \text { Fraction Leached }\end{gathered}$

$\begin{array}{lll}1 & 8.4 \times 10^{-6} & 1.7 \times 10^{-5} \\ 2 & 8.1 \times 10^{-6} & 3.4 \times 10^{-5} \\ 3 & 6.1 \times 10^{-6} & 4.7 \times 10^{-5} \\ 4 & 9.7 \times 10^{-6} & 6.7 \times 10^{-5} \\ 5 & 4.8 \times 10^{-6} & 1.1 \times 10^{-4} \\ 6 & 3.7 \times 10^{-6} & 1.5 \times 10^{-4} \\ 7 & 6.4 \times 10^{-7} & 2.5 \times 10^{-4} \\ 8 & 7.4 \times 10^{-6} & 3.6 \times 10^{-4} \\ 9 & 5.7 \times 10^{-6} & 4.4 \times 10^{-4} \\ 10 & 6.1 \times 10^{-6} & 5.3 \times 10^{-4} \\ 11 & 7.2 \times 10^{-6} & 6.3 \times 10^{-4} \\ 12 & 7.8 \times 10^{-6} & 7.5 \times 10^{-4} \\ 13 & 3.2 \times 10^{-6} & 9.5 \times 10^{-4} \\ 14 & 3.7 \times 10^{-6} & 1.2 \times 10^{-3} \\ 15 & 4.1 \times 10^{-6} & 1.5 \times 10^{-3} \\ 16 & 5.2 \times 10^{-6} & 1.8 \times 10^{-3} \\ 17 & 6.7 \times 10^{-6} & 2.2 \times 10^{-3} \\ 18 & 5.4 \times 10^{-6} & 2.5 \times 10^{-3} \\ 19 & 1.2 \times 10^{-6} & 3.0 \times 10^{-3} \\ 20 & 9.0 \times 10^{-7} & 3.4 \times 10^{-3}\end{array}$




\begin{tabular}{|c|c|c|}
\hline \multirow[b]{2}{*}{ Series \# } & \multicolumn{2}{|c|}{$239+240$ Pu-Top Section } \\
\hline & $\begin{array}{r}\text { Leach Rate } \\
\mathrm{g} / \mathrm{cm}^{2}-\text { day }\end{array}$ & $\begin{array}{l}\text { Cumulative } \\
\text { Fraction Leached } \\
\end{array}$ \\
\hline 1 & $1.8 \times 10^{-5}$ & $4.8 \times 10^{-5}$ \\
\hline 2 & $1.0 \times 10^{-6}$ & $5.1 \times 10^{-5}$ \\
\hline 3 & $2.3 \times 10^{-6}$ & $5.7 \times 10^{-5}$ \\
\hline 4 & $1.7 \times 10^{-6}$ & $6.1 \times 10^{-5}$ \\
\hline 5 & $1.2 \times 10^{-6}$ & $7.4 \times 10^{-5}$ \\
\hline 6 & $1.5 \times 10^{-6}$ & $9.8 \times 10^{-5}$ \\
\hline 7 & $1.6 \times 10^{-6}$ & $1.3 \times 10^{-4}$ \\
\hline 8 & $1.8 \times 10^{-6}$ & $1.6 \times 10^{-4}$ \\
\hline 9 & $1.8 \times 10^{-6}$ & $2.0 \times 10^{-4}$ \\
\hline 10 & $2.2 \times 10^{-6}$ & $2.4 \times 10^{-4}$ \\
\hline 11 & $3.5 \times 10^{-6}$ & $3.0 \times 10^{-4}$ \\
\hline 12 & $3.4 \times 10^{-6}$ & $3.6 \times 10^{-4}$ \\
\hline 13 & $1.4 \times 10^{-6}$ & $4.7 \times 10^{-4}$ \\
\hline 14 & $1.2 \times 10^{-6}$ & $5.7 \times 10^{-4}$ \\
\hline 15 & $1.4 \times 10^{-6}$ & $7.0 \times 10^{-4}$ \\
\hline 16 & $1.6 \times 10^{-6}$ & $8.4 \times 10^{-4}$ \\
\hline 17 & $1.4 \times 10^{-6}$ & $9.4 \times 10^{-4}$ \\
\hline 18 & $1.4 \times 10^{-6}$ & $1.0 \times 10^{-3}$ \\
\hline 19 & $2.5 \times 10^{-7}$ & $1.2 \times 10^{-3}$ \\
\hline 20 & $1.4 \times 10^{-7}$ & $1.2 \times 10^{-3}$ \\
\hline
\end{tabular}




\begin{tabular}{|c|c|c|}
\hline \multirow[b]{2}{*}{ Series \# } & \multicolumn{2}{|c|}{${ }^{242} \mathrm{Cm}$-Bottom Section } \\
\hline & $\begin{array}{l}\text { Leach Rate } \\
\mathrm{g} / \mathrm{cm}^{2} \text {-day }\end{array}$ & $\begin{array}{c}\text { Cumulative } \\
\text { Fraction Leached }\end{array}$ \\
\hline 1 & $1.5 \times 10^{-6}$ & $2.4 \times 10^{-6}$ \\
\hline 2 & $2.9 \times 10^{-7}$ & $2.8 \times 10^{-6}$ \\
\hline 3 & $2.4 \times 10^{-7}$ & $3.2 \times 10^{-6}$ \\
\hline 4 & $2.5 \times 10^{-7}$ & $3.6 \times 10^{-6}$ \\
\hline 5 & $9.6 \times 10^{-8}$ & $4.2 \times 10^{-6}$ \\
\hline 6 & $1.1 \times 10^{-7}$ & $5.3 \times 10^{-6}$ \\
\hline 7 & $1.1 \times 10^{-7}$ & $6.5 \times 10^{-6}$ \\
\hline 8 & $8.7 \times 10^{-8}$ & $7.5 \times 10^{-6}$ \\
\hline 9 & $8.1 \times 10^{-8}$ & $8.4 \times 10^{-6}$ \\
\hline 10 & $6.0 \times 10^{-8}$ & $9.0 \times 10^{-6}$ \\
\hline 11 & $9.9 \times 10^{-8}$ & $1.0 \times 10^{-5}$ \\
\hline 12 & $1.4 \times 10^{-7}$ & $1.2 \times 10^{-5}$ \\
\hline 13 & $4.0 \times 10^{-8}$ & $1.4 \times 10^{-5}$ \\
\hline 14 & $1.1 \times 10^{-7}$ & $1.9 \times 10^{-5}$ \\
\hline 15 & $1.4 \times 10^{-7}$ & $2.6 \times 10^{-5}$ \\
\hline 16 & $1.4 \times 10^{-7}$ & $3.3 \times 10^{-5}$ \\
\hline 17 & $2.2 \times 10^{-7}$ & $4.3 \times 10^{-5}$ \\
\hline 18 & $2.7 \times 10^{-7}$ & $5.5 \times 10^{-5}$ \\
\hline 19 & $3.3 \times 10^{-7}$ & $1.5 \times 10^{-4}$ \\
\hline 20 & $6.2 \times 10^{-8}$ & $1.7 \times 10^{-4}$ \\
\hline
\end{tabular}




\section{${ }^{242} \mathrm{Cm}$-Lower-Middle Section}

Series \# $\quad \begin{array}{rc}\text { Leach Rate } \\ \mathrm{g} / \mathrm{cm}^{2} \text {-day }\end{array} \quad \begin{gathered}\text { Cumulative } \\ \text { Fraction Leached }\end{gathered}$

\begin{tabular}{rll}
1 & $8.5 \times 10^{-7}$ & $1.9 \times 10^{-6}$ \\
2 & $1.5 \times 10^{-7}$ & $2.3 \times 10^{-6}$ \\
3 & $1.2 \times 10^{-7}$ & $2.5 \times 10^{-6}$ \\
4 & $1.0 \times 10^{-7}$ & $2.8 \times 10^{-6}$ \\
5 & $7.6 \times 10^{-8}$ & $3.5 \times 10^{-6}$ \\
6 & $1.1 \times 10^{-7}$ & $4.9 \times 10^{-6}$ \\
7 & $7.6 \times 10^{-8}$ & $6.1 \times 10^{-6}$ \\
8 & $7.4 \times 10^{-8}$ & $7.3 \times 10^{-6}$ \\
9 & $8.3 \times 10^{-8}$ & $8.6 \times 10^{-6}$ \\
10 & $8.1 \times 10^{-8}$ & $9.9 \times 10^{-6}$ \\
$* 11$ & $7.9 \times 10^{-8}$ & $1.1 \times 10^{-5}$ \\
12 & $2.5 \times 10^{-7}$ & $1.5 \times 10^{-5}$ \\
13 & $5.9 \times 10^{-8}$ & $1.9 \times 10^{-5}$ \\
14 & $1.3 \times 10^{-7}$ & $2.8 \times 10^{-5}$ \\
15 & $1.5 \times 10^{-7}$ & $4.0 \times 10^{-5}$ \\
16 & $2.3 \times 10^{-7}$ & $5.7 \times 10^{-5}$ \\
17 & $3.9 \times 10^{-7}$ & $8.2 \times 10^{-5}$ \\
18 & $6.8 \times 10^{-7}$ & $1.3 \times 10^{-4}$ \\
19 & $1.4 \times 10^{-7}$ & $1.8 \times 10^{-4}$ \\
20 & $1.7 \times 10^{-7}$ & $2.6 \times 10^{-4}$ \\
\hline * Denotes that an analysis is not avai1able \\
for this series. The number is an average \\
of the above series with the same 1 each time.
\end{tabular}




\begin{tabular}{ccc} 
& \multicolumn{2}{c}{${ }^{242}$ Cm-Upper-Middle Section } \\
\cline { 2 - 3 } Series \# & $\begin{array}{c}\text { Leach Rate } \\
\mathrm{g} / \mathrm{cm}^{2} \text {-day }\end{array}$ & $\begin{array}{c}\text { Cumulative } \\
\text { Fraction Leached }\end{array}$ \\
\cline { 3 - 3 } 1 & $9.8 \times 10^{-7}$ & $2.0 \times 10^{-6}$ \\
2 & $4.1 \times 10^{-7}$ & $2.9 \times 10^{-6}$ \\
3 & $3.3 \times 10^{-7}$ & $3.6 \times 10^{-6}$ \\
4 & $2.7 \times 10^{-7}$ & $4.2 \times 10^{-6}$ \\
5 & $6.6 \times 10^{-8}$ & $4.7 \times 10^{-6}$ \\
6 & $1.1 \times 10^{-7}$ & $6.1 \times 10^{-6}$ \\
7 & $1.4 \times 10^{-7}$ & $8.2 \times 10^{-6}$ \\
8 & $1.7 \times 10^{-7}$ & $1.1 \times 10^{-5}$ \\
9 & $1.5 \times 10^{-7}$ & $1.3 \times 10^{-5}$ \\
10 & $1.2 \times 10^{-7}$ & $1.5 \times 10^{-5}$ \\
11 & $7.2 \times 10^{-8}$ & $1.6 \times 10^{-5}$ \\
12 & $7.6 \times 10^{-8}$ & $1.7 \times 10^{-5}$ \\
13 & $6.5 \times 10^{-8}$ & $2.1 \times 10^{-5}$ \\
14 & $5.9 \times 10^{-8}$ & $2.5 \times 10^{-5}$ \\
15 & $1.4 \times 10^{-7}$ & $3.4 \times 10^{-5}$ \\
16 & $2.7 \times 10^{-7}$ & $5.2 \times 10^{-5}$ \\
17 & $3.2 \times 10^{-7}$ & $7.2 \times 10^{-5}$ \\
18 & $3.5 \times 10^{-7}$ & $9.2 \times 10^{-5}$ \\
19 & $1.8 \times 10^{-7}$ & $1.8 \times 10^{-4}$ \\
20 & $6.7 \times 10^{-8}$ & $2.1 \times 10^{-4}$
\end{tabular}




\begin{tabular}{|c|c|c|}
\hline \multirow[b]{2}{*}{ Series \# } & \multicolumn{2}{|c|}{${ }^{242} \mathrm{Cm}$-Top Section } \\
\hline & $\begin{array}{r}\text { Leach Rate } \\
\mathrm{g} / \mathrm{cm}^{2}-\text { day }\end{array}$ & $\begin{array}{c}\text { Cumulative } \\
\text { Fraction Leached }\end{array}$ \\
\hline 1 & $2.4 \times 10^{-6}$ & $6.3 \times 10^{-6}$ \\
\hline 2 & $6.9 \times 10^{-7}$ & $8.1 \times 10^{-6}$ \\
\hline 3 & $4.0 \times 10^{-7}$ & $9.2 \times 10^{-6}$ \\
\hline 4 & $4.9 \times 10^{-7}$ & $1.1 \times 10^{-5}$ \\
\hline 5 & $2.8 \times 10^{-7}$ & $1.3 \times 10^{-5}$ \\
\hline 6 & $1.7 \times 10^{-7}$ & $1.6 \times 10^{-5}$ \\
\hline 7 & $2.1 \times 10^{-7}$ & $2.0 \times 10^{-5}$ \\
\hline 8 & $2.7 \times 10^{-7}$ & $2.5 \times 10^{-5}$ \\
\hline 9 & $8.0 \times 10^{-8}$ & $2.7 \times 10^{-5}$ \\
\hline 10 & $1.3 \times 10^{-7}$ & $2.9 \times 10^{-5}$ \\
\hline 11 & $1.4 \times 10^{-7}$ & $3.1 \times 10^{-5}$ \\
\hline 12 & $1.7 \times 10^{-7}$ & $3.5 \times 10^{-5}$ \\
\hline 13 & $2.4 \times 10^{-8}$ & $3.7 \times 10^{-5}$ \\
\hline 14 & $5.6 \times 10^{-8}$ & $4.1 \times 10^{-5}$ \\
\hline 15 & $5.9 \times 1 p^{-8}$ & $4.6 \times 10^{-5}$ \\
\hline 16 & $1.1 \times 10^{-7}$ & $5.5 \times 10^{-5}$ \\
\hline 17 & $1.7 \times 10^{-7}$ & $6.9 \times 10^{-5}$ \\
\hline 18 & $2.4 \times 10^{-7}$ & $8.6 \times 10^{-5}$ \\
\hline 19 & $9.8 \times 10^{-8}$ & $1.3 \times 10^{-4}$ \\
\hline 20 & $6.6 \times 10^{-8}$ & $1.9 \times 10^{-4}$ \\
\hline
\end{tabular}




\begin{tabular}{|c|c|c|}
\hline \multirow[b]{2}{*}{ Series \# } & \multicolumn{2}{|c|}{${ }^{244}$ Cm-Bottom Section } \\
\hline & $\begin{array}{l}\text { Leach Rate } \\
\mathrm{g} / \mathrm{cm}^{2} \text {-day }\end{array}$ & $\begin{array}{c}\text { Cumulative } \\
\text { Fraction Leached } \\
\end{array}$ \\
\hline 1 & $1.5 \times 10^{-6}$ & $2.3 \times 10^{-6}$ \\
\hline 2 & $3.0 \times 10^{-7}$ & $2.8 \times 10^{-6}$ \\
\hline 3 & $2.1 \times 10^{-7}$ & $3.1 \times 10^{-6}$ \\
\hline 4 & $2.0 \times 10^{-7}$ & $3.4 \times 10^{-6}$ \\
\hline 5 & $1.0 \times 10^{-7}$ & $4.1 \times 10^{-6}$ \\
\hline 6 & $8.7 \times 10^{-8}$ & $4.9 \times 10^{-6}$ \\
\hline 7 & $9.6 \times 10^{-8}$ & $6.0 \times 10^{-6}$ \\
\hline 8 & $9.8 \times 10^{-8}$ & $7.1 \times 10^{-6}$ \\
\hline 9 & $7.7 \times 10^{-8}$ & $7.9 \times 10^{-6}$ \\
\hline 10 & $7.5 \times 10^{-8}$ & $8.7 \times 10^{-6}$ \\
\hline 11 & $1.1 \times 10^{-7}$ & $1.0 \times 10^{-5}$ \\
\hline 12 & $1.5 \times 10^{-7}$ & $1.2 \times 10^{-5}$ \\
\hline 13 & $4.4 \times 10^{-8}$ & $1.4 \times 10^{-5}$ \\
\hline 14 & $1.0 \times 10^{-7}$ & $1.8 \times 10^{-5}$ \\
\hline 15 & $1.5 \times 10^{-7}$ & $2.6 \times 10^{-5}$ \\
\hline 16 & $1.3 \times 10^{-7}$ & $3.3 \times 10^{-5}$ \\
\hline 17 & $2.2 \times 10^{-7}$ & $4.3 \times 10^{-5}$ \\
\hline 18 & $2.7 \times 10^{-7}$ & $5.5 \times 10^{-5}$ \\
\hline 19 & $2.6 \times 10^{-7}$ & $1.3 \times 10^{-4}$ \\
\hline 20 & $5.5 \times 10^{-8}$ & $1.5 \times 10^{-4}$ \\
\hline
\end{tabular}




\begin{tabular}{ccc} 
& \multicolumn{2}{c}{${ }^{244}$ Cm-Lower-Middle Section } \\
\cline { 2 - 3 } Series \# & $\begin{array}{c}\text { Leach Rate } \\
\mathrm{g} / \mathrm{cm}^{2} \text {-day }\end{array}$ & $\begin{array}{c}\text { Cumulative } \\
\text { Fraction Leached }\end{array}$ \\
\cline { 2 - 3 } 1 & & \\
2 & $8.7 \times 10^{-7}$ & $2.0 \times 10^{-6}$ \\
3 & $1.6 \times 10^{-7}$ & $2.3 \times 10^{-6}$ \\
4 & $1.5 \times 10^{-7}$ & $2.7 \times 10^{-6}$ \\
5 & $9.1 \times 10^{-8}$ & $2.9 \times 10^{-6}$ \\
6 & $8.6 \times 10^{-8}$ & $3.7 \times 10^{-6}$ \\
7 & $1.1 \times 10^{-7}$ & $5.2 \times 10^{-6}$ \\
8 & $7.9 \times 10^{-8}$ & $6.4 \times 10^{-6}$ \\
9 & $8.1 \times 10^{-8}$ & $7.7 \times 10^{-6}$ \\
10 & $7.9 \times 10^{-8}$ & $9.0 \times 10^{-6}$ \\
11 & $8.8 \times 10^{-8}$ & $1.0 \times 10^{-5}$ \\
12 & $8.2 \times 10^{-8}$ & $1.2 \times 10^{-5}$ \\
13 & $2.7 \times 10^{-7}$ & $1.6 \times 10^{-5}$ \\
14 & $6.7 \times 10^{-8}$ & $2.1 \times 10^{-5}$ \\
15 & $1.1 \times 10^{-7}$ & $2.8 \times 10^{-5}$ \\
16 & $1.6 \times 10^{-7}$ & $4.1 \times 10^{-5}$ \\
17 & $2.5 \times 10^{-7}$ & $5.9 \times 10^{-5}$ \\
18 & $3.8 \times 10^{-7}$ & $8.4 \times 10^{-5}$ \\
19 & $5.9 \times 10^{-7}$ & $1.2 \times 10^{-4}$ \\
20 & $1.5 \times 10^{-7}$ & $1.8 \times 10^{4}$ \\
& $1.3 \times 10^{-7}$ & $2.5 \times 10^{-4}$
\end{tabular}




\begin{tabular}{ccc} 
Series \# & \multicolumn{2}{l}{${ }^{244}$ Cm-Upper-Middle Section } \\
\cline { 2 - 3 } & $\begin{array}{c}\text { Leach Rate } \\
\mathrm{g} / \mathrm{cm}^{2} \text {-day }\end{array}$ & $\begin{array}{c}\text { Cumulative } \\
\text { Fraction Leached }\end{array}$ \\
1 & $9.9 \times 10^{-7}$ & $2.1 \times 10^{-6}$ \\
2 & $3.7 \times 10^{-7}$ & $2.8 \times 10^{-6}$ \\
3 & $2.8 \times 10^{-7}$ & $3.4 \times 10^{-6}$ \\
4 & $2.2 \times 10^{-7}$ & $3.9 \times 10^{-6}$ \\
5 & $1.0 \times 10^{-7}$ & $4.7 \times 10^{-6}$ \\
6 & $1.2 \times 10^{-7}$ & $6.2 \times 10^{-6}$ \\
7 & $1.3 \times 10^{-7}$ & $8.2 \times 10^{-6}$ \\
8 & $1.6 \times 10^{-7}$ & $1.1 \times 10^{-5}$ \\
9 & $1.6 \times 10^{-7}$ & $1.3 \times 10^{-5}$ \\
10 & $1.1 \times 10^{-7}$ & $1.4 \times 10^{-5}$ \\
11 & $8.1 \times 10^{-8}$ & $1.6 \times 10^{-5}$ \\
12 & $6.8 \times 10^{-8}$ & $1.7 \times 10^{-5}$ \\
13 & $6.2 \times 10^{-8}$ & $2.1 \times 10^{-5}$ \\
14 & $6.6 \times 10^{-8}$ & $2.5 \times 10^{-5}$ \\
15 & $1.1 \times 10^{-7}$ & $3.3 \times 10^{-5}$ \\
16 & $2.8 \times 10^{-7}$ & $5.1 \times 10^{-5}$ \\
17 & $3.1 \times 10^{-7}$ & $7.0 \times 10^{-5}$ \\
18 & $3.5 \times 10^{-7}$ & $9.0 \times 10^{-5}$ \\
19 & $1.0 \times 10^{-7}$ & $1.3 \times 10^{-4}$ \\
20 & $5.7 \times 10^{-8}$ & $1.5 \times 10^{-4}$
\end{tabular}




\begin{tabular}{|c|c|c|}
\hline \multirow[b]{2}{*}{ Series \# } & \multicolumn{2}{|c|}{${ }^{244} \mathrm{Cm}$-Top Section } \\
\hline & $\begin{array}{r}\text { Leach Rate } \\
\mathrm{g} / \mathrm{cm}^{2}-\text { day } \\
\end{array}$ & $\begin{array}{c}\text { Cumulative } \\
\text { Fraction Leached } \\
\end{array}$ \\
\hline 1 & $2.6 \times 10^{-6}$ & $6.8 \times 10^{-6}$ \\
\hline 2 & $5.9 \times 10^{-7}$ & $8.4 \times 10^{-6}$ \\
\hline 3 & $4.4 \times 10^{-7}$ & $9.5 \times 10^{-6}$ \\
\hline 4 & $5.5 \times 10^{-7}$ & $1.1 \times 10^{-5}$ \\
\hline 5 & $2.7 \times 10^{-7}$ & $1.4 \times 10^{-5}$ \\
\hline 6 & $1.8 \times 10^{-7}$ & $1.7 \times 10^{-5}$ \\
\hline 7 & $2.7 \times 10^{-7}$ & $2.1 \times 10^{-5}$ \\
\hline 8 & $2.2 \times 10^{-7}$ & $2.5 \times 10^{-5}$ \\
\hline 9 & $1.4 \times 10^{-7}$ & $2.7 \times 10^{-5}$ \\
\hline 10 & $1.3 \times 10^{-7}$ & $3.0 \times 10^{-5}$ \\
\hline 11 & $1.4 \times 10^{-7}$ & $3.2 \times 10^{-5}$ \\
\hline 12 & $1.7 \times 10^{-7}$ & $3.5 \times 10^{-5}$ \\
\hline 13 & $2.7 \times 10^{-8}$ & $3.8 \times 10^{-5}$ \\
\hline 14 & $5.0 \times 10^{-8}$ & $4.2 \times 10^{-5}$ \\
\hline 15 & $5.7 \times 10^{-8}$ & $4.7 \times 10^{-5}$ \\
\hline 16 & $1.1 \times 10^{-7}$ & $5.6 \times 10^{-5}$ \\
\hline 17 & $1.4 \times 10^{-7}$ & $6.7 \times 10^{-5}$ \\
\hline 18 & $2.1 \times 10^{-7}$ & $8.2 \times 10^{-5}$ \\
\hline 19 & $3.0 \times 10^{-8}$ & $9.6 \times 10^{-5}$ \\
\hline 20 & $6.7 \times 10^{-8}$ & $1.3 \times 10^{-4}$ \\
\hline
\end{tabular}




\section{DISTRIBUTION}

No. of

Copies

\section{UNITED STATES}

A. A. Churm

DOE Chicago Patent Group

9800 South Cass Avenue

Argonne, IL 60439

W. G. Belter

DOE Division of Biomedical and Environmental Research

Earth Sciences Branch

Washington, DC 20545

W. E. Mott

DOE Division of Environmental

Control Technology

Washington, DC 20545

R. W. Ramsey

DOE Division of Environmental

Control Technology

washington, DC 20545

D. W. Readey

DOE Division of Physical Research

Washington, DC 20545

C. R. Cooley

DOE Division of Waste Management Production and Reprocessing

Washington, DC 20545

G. W. Cunningham

DOE Division of Waste Management

Production and Reprocessing

Washington, DC 20545

G. H. Daly

DOE Division of Waste

Management Production

and Reprocessing

Washington, DC 20545
No. of

Copies

C. H. George

DOE Division of Waste

Management Production

and Reprocessing

Washington, DC 20545

L. Harmon

DOE Office of Waste Management

Washington, DC 20545

C. A. Heath

DOE Division of Waste Isolation

Washington, DC 20545

C. W. Kuhlman

DOE Division of Planning and Analys is

Washington, DC 20545

M. J. Lawrence

DOE Division of Fuel Storage and Transfer

Washington, DC 20545

J. Leary

DOE Division of Waste

Management Production

and Reprocessing

Washington, DC 20545

D. Leclaire

DOE Division of Budget and

Administration

Washington, DC 20545

$S$. Meyers

DOE Office of Waste Management

Washington, DC 20545

G. 0ertel

DOE Division of Waste Products Washington, DC 20545 
No. of

Copies

R. Romatowski

DOE Office of Waste Management

Washington, DC 20545

\section{A. F. Perge \\ DOE Division of Waste Management \\ Production and Reprocessing \\ Washington, DC 20545 \\ R. Stein \\ DOE Office of Waste Management \\ Washington, DC 20545}

J. M. Taub

DOE Division of Waste Mangement

Production and Reprocessing

Washington, DC 20545

R. D. Walton

DOE Division of Waste Management

Production and Reprocessing

Washington, DC 20545

D. L. Vieth

DOE Division of Waste Management Production and Reprocessing

Washington, DC 20545

C. A. Hawley, Jr.,

DOE Idaho Operations Office

P. 0. Box 2108

Idaho Fal1s, ID 83401

D. E. Large

DOE Oak Ridge Operations Office

P. 0. Box X

Oak Ridge, TN 37830

J. J. Schreiber

DOE Oak Ridge Operations Office

P. 0. Box X

Oak Ridge, TN 37830

E. S. Goldberg

DOE Savannah River Operations office

P. 0. Box A

Aiken, SC 29801
No. of

Copies

267 DOE Technical Information Center

Harry Lawroski

A1lied Chemical Corporation

550 Second Street

Idaho Falls, ID 8340 ]

B. R. Dickey

Allied Chemical Corporation

550 Second Street

Idaho Falls, ID 83401

J. A. Buckham

Allied-General Nuclear Service

P. 0. Box 847

Barnwel1, SC 29812

W. F. Godfrey

Allied General Nuclear Service P. 0. Box 847

Barnwe11, SC 29812

A. Williams

Allied General Nuclear Service P. 0. Box 847

Barnwe11, SC 29812

L. Jardine

Argonne National Laboratory

9700 South Cass Avenue

Argonne, IL 60439

M. M. Steindler

Argonne National Laboratory

9700 South Cass Avenue

Argonne, IL 60439

M. F. Browning

Battelle Columbus Laboratories 505 King Avenue

Columbus, $\mathrm{OH} 43201$

J. 0. Duguid

Battelle Columbus Laboratories

505 King Avenue

Columbus, $\mathrm{OH} 43201$ 
No. of

Copies

J. F. Kircher

Battelle Columbus Laboratories 505 King Avenue

Columbus, $\mathrm{OH} 43201$

Bechtel Corporation

50 Beale Street

San Francisco, CA 94119

M. Steinberg

Brookhaven National Laboratory

Upton, Long Island, NY 11973

R. Beckmann

Combustion Engineering, Inc.

Combustion Division

Windsor, CT 06095

M. G. Britton

Corning Glass Works

Technical Staffs Division

Corning, NY 14830

D. L. Ziegler

Dow Chemical Company (DOE)

Rocky Flats Division

P. 0. Box 888

Golden, C0 80401

L. H. Meyer

E. I. duPont deNemours and Co. Savannah River Laboratory

Aiken, SC 29801

R. F. Bradley

E. I. duPont deNemours and Co. Savannah River Laboratory

Aiken, SC 29801

C. H. Ice

E. I. dePont deNemours and Co.

Savannah River Laboratory

Aiken, SC 29801

A. S. Jennings

E. I. duPont deNemours and Co. Savannah River Laboratory

Aiken, SC 29801
No. of

Copies

R. F. Williams

Electric Power Research Institute 3412 Hillview Avenue

P. 0. Box 10412

Palo Alto, CA 94304

G. L. Meyer

Environmental Protection Agency

Technology Assessment Division

(AW-559)

Office of Radiation Programs

Washington, DC 20460

R. G. Barnes

General Electric Company

175 Curtner Avenue (M/C 160)

San Jose, CA 95125

A. Carson

General Electric Company

175 Curtner Avenue (M/C 160)

San Jose, CA 95138

L. H. Brooks

General Atomic Company

P. 0. Box 81608

San Diego, CA 92138

J. J. Shefcik

General Atomic Company

P. 0. Box 81608

San Diego, CA 92138

C. W. Christenson

Los Alamos Scientific Laboratory (DOE)

P. 0. Box 1663

Los Alamos, NM 87544

G. A. Cowan

CNC Division

Los Alamos Scientific Laboratory

P. 0. Box 1663

Los Alamos, NM 87545 
No. of

Copies

John Pomeroy

Technical Secretary

National Academy of Sciences

Committee of Radioactive Waste

Mana gement

National Research Council

2101 Constitution Avenue

Washington, DC 20418

J. P. Duckworth

Plant Manager

Nuclear Fuel Services, Inc.

P. 0. Box 124

West Valley, NY 14171

W. Lewis, Vice President

Nuclear Fuel Services, Inc.

6000 Executive Blvd., Suite 600

Rockville, MD 20852

R. B. Chitwood

NRC Directorate of Licensing for Fuels and Materials

4915 St. Elmo Avenue

Bethesda, MD 20014

S. M. Smiley

NRC Directorate of Licensing

for Fuels and Materials

4915 St. Elmo Avenue

Bethesda, MD 20014

W. P. Bishop

NRC Division of Materials and

Fuel Cycle Facility Licensing

Washington, DC 20555

2 Oak Ridge National Laboratory (DOE)

Central Research Library

Document Reference Section

P. 0. Box $X$

Oak Ridge, TN 37830

John Van Clive

Oak Ridge National Laboratory

Bldg. $7601 \quad X-10$

Oak Ridge, TN 37830
No. of

Copies

J. G. Cline

General Manager

NYS Atomic and Space Development Authority

230 Park Avenue, Rm. 2425

New York, NY 10017

2 Oak Ridge National Laboratory (DOE)

Laboratory Records Dept., ORNL-RC

P. 0. BOX X

Oak Ridge, TN 37830

G. J. McGarthy

Pennsylvania State Universtiy

Materials Research Laboratory

University Park, PA 16802

M. A. Thompson

Rockwell International

Rocky Flats Division

Golden, CO 80401

R. W. Lynch

Sandia Laboratories

Albuquerque, NM 87107

P. 0. O'Brien

Sandia Laboratories

Albuquerque, NM 87107

W. Weart

Sandia Laboratories

Albuquerque, NM 87107

T. R. Simpson

TWR Defense and Space Systems Group

Technical Information Center S-1930

One Space Park

Redondo Beach, CA 90278

J. 0. Blomeke

Union Carbide Corporation (ORNL)

Chemical Technology Divison

P. 0. Box $Y$

Oak Ridge, TN 37830 
No. of

Copies

H. W. Godbee

Union Carbide Corporation (ORNL)

Chemical Technology Division

P. 0. Box $Y$

Oak Ridge, TN 37830

C. D. Zerby

Union Carbide Corporation (ORNL)

Chemical Technology Division

Office of Waste Isolation

P. 0. Box $Y$

Oak Ridge, TN 37830

R. G. Post

College of Engineering

University of Arizona

Tucson, AZ 85721

\section{FOREIGN}

Dr. Piero Risolute'

Head, Radioactive Waste Program

Plant Engineering Dept.

Agip Nucleare

Milano

C. so di Porta Romana, 68

ITALY

P. J. Dyne

Atomic Energy of Canada, Ltd.

W.N.R.E. Pinawa, Manitoba

ROE 1LO

CANADA

J. A. C. Marples

Atomic Energy Research

Establ ishment

Harwe 11, Didcot, Berks

ENGLAND

N. S. Suder Rajan

Bhabha Atomic Research Centre

Government of India

Hal1 No. 5

Trobay

Bombay 8S

INDIA
No. of

Copies

R. P. Rand1

Bundeministerium fur Bildung und Wessenschaft

D53 Bonn 12

Postfach 120124

GERMANY

Bundeministerium für Forschung Und Technologie

Stressemannstrasse 2

5300 Bonn

GERMANY

Dr. Mrs. Bel1

Center for Atomic Energy Documentation (ZAED)

P. 0. Box 3640

7500 Karlsruhe

GERMANY

2 R. Bonniaud

Centre de Marcoule

B. P. 106

30 - Bagnols S/Ceze

FRANCE

F. Gera

CNEN

CSN Casaccia L.I.S.

C.P. 2400, 00100

Rome

ITALY

Dr. W. Heimer 1

Gelsenberg Aktiengese11schaft

Entwicklungsgruppe Julich

c/o Kernforschungsanlage (ICT)

517 Julich 1, Postfach 1913

GERMANY

Gesellschaft für

Wiedenaufarbeitung

von Kernbrennstoffen mbH (GWK)

7301 Leopoldshafen

GERMANY 
No. of

Copies

Gesellschaft für Kernforschung (Gfk)

Postfach 3640

7500 Karlsruhe

GERMANY

Hans W. Levi

Han-Meitner Insitut

1 Berlin 39

Glienickerstr. 100

GERMANY

W. Lutze

Hahn-Meitner Institut

1 Berlin 39

Glienickerstr. 100

GERMANY

E. R. Merz..

Insitute fur Chemische

Technologie

Kernforschungsanloge Julich GmbH

D517 Julich

Postfach 365

GERMANY

K. H. Rattay

Institut für Chemische

Technologie

Kernforschungsanloge Julich $\mathrm{GmbH}$

D517 Julich

Postfach 365

GERMANY

2 International Atomic Energy Agency

Kartner Ring 11

P. 0. Box 590

A1011, Vienna

AUSTRIA

KFA-Kernforschungsanlage

Julich GmbH

Postfach 1913

5170 Julich 1

GERMANY
No. of

Copies

Willy Bocola

Laboratorio di Ingegneria

Sanitaria

Via Anguillarese km $1+300$

Roma

ITALY

2 W. Krause

Nuclear Research Center

Waste Management Dept.

D75 Karlsruhe

Weberstr. 5

GERMANY

Dr. Hartmut Witte

NUKEM GmbH

Postfach 110080

6540 Hanau 11

GERMANY

T. Sakakura

Power Research and Nuclear

Fuel Development Division

Reprocessing Division

PNL (1-9-13, Alasaka Minato-Ku, Tokyo)

JAPAN

Dr. H. F. Ramdohr

c/o Friedrich Unde GmbH

46 Durtmund

Gegginstre 10-12

GERMANY

J. H. C. Castro

Universidade Federal de San Carlos

Department de Engenharia de Materials

13560 Sao Carolos, S.P.

BRAZIL

D. W. Clelland

United Kingdom Atomic

Energy Authority

Risley

ENGLAND 
No. of

Copies

ONSITE

DOE Richland Operations Office

1 Programs Division

5 Nuclear Fuel Cycle and

Production Division

0 . Elgert

R. B. Goranson

H. E. Ransom

D. J. Squires

M. J. Zamorski

Assistant Manager for

Technical Operations -

F. R. Standerfer

1 Safety and Quality Assurance

Division

J. H. Straub, Director

8 Rockwell Hanford Operations
R. J. Gimera
D. R. Gustavson
R. E. Issacson
E. J. Kosiancic
I. E. Reep
J. H. Roecker
W. W. Schulz
D. D. Wodrich

3 Exxon Nuclear Company
S. J. Beard
L. T. Lakey
M. E. Spaeth

1 Joint Center for Graduate Study

L. C. 01 sen

1 Westinghouse Hanford Company

G. L. Richardson
No. of

Copies

65 Pacific Northwest Laboratory

T. W. Ambrose

D. N. Berger

W. J. Bjorklund

H. T. Blair

W. F. Bonner

D. J. Bradley (10)

J. L. Buelt

H. C. Burkholder

N. E. Carter

C. C. Chapman

T. D. Chikalla

M. 0. Cloninger

R. D. Dierks

J. W. Finnigan

A. A. Garrett

M. S. Hanson

J. C. Hartl

O. F. Hill

L. R. Jaech

J. H. Jarrett

Y. B. Katayama

W. S. Kelly

R. S. Kemper

D. E. Knowlton

D. E. Larson

J. M. Lukacs

R. P. Marshal1

J. L. McElroy

J. S. McPherson

G. B. Mellinger

J. E. Mendel

R. E. Nightingale

D. E. Olesen

C. R. Palmer

A. M. Platt

D. L. Prezbindowski (2)

F. P. Roberts

L. S. Romero

W. A. Ross

J. M. Rusin

D. H. Siemens

S. C. Slate

R. L. Treat

R. P. Turcotte

H. H. Van Tuyl 
No. of

Copies

J. H. Westsik, Jr.

L. D. Williams

W. K. Winegardner

Technical Information (5)

Publishing Coordination (2) 